\title{
First Order Algorithms in Variational Image Processing
}

\author{
M. Burger, A. Sawatzky*, and G. Steidl ${ }^{\dagger}$
}

December 16, 2014

\section{Introduction}

Variational methods in imaging are nowadays developing towards a quite universal and flexible tool, allowing for highly successful approaches on tasks like denoising, deblurring, inpainting, segmentation, super-resolution, disparity, and optical flow estimation. The overall structure of such approaches is of the form

$$
\mathcal{D}(K u)+\alpha \mathcal{R}(u) \rightarrow \min _{u}
$$

where the functional $\mathcal{D}$ is a data fidelity term also depending on some input data $f$ and measuring the deviation of $K u$ from such and $\mathcal{R}$ is a regularization functional. Moreover $K$ is a (often linear) forward operator modeling the dependence of data on an underlying image, and $\alpha$ is a positive regularization parameter. While $\mathcal{D}$ is often smooth and (strictly) convex, the current practice almost exclusively uses nonsmooth regularization functionals. The majority of successful techniques is using nonsmooth and convex functionals like the total variation and generalizations thereof, cf. [28, 31, 40], or $\ell_{1}$-norms of coefficients arising from scalar products with some frame system, cf. [73] and references therein.

The efficient solution of such variational problems in imaging demands for appropriate algorithms. Taking into account the specific structure as a sum of two very different terms to be minimized, splitting algorithms are a quite canonical choice. Consequently this field has revived the interest in techniques like operator splittings or augmented Lagrangians. In this chapter we shall provide an overview of methods currently developed and recent results as well as some computational studies providing a comparison of different methods and also illustrating their success in applications.

We start with a very general viewpoint in the first sections, discussing basic notations, properties of proximal maps, firmly non-expansive and averaging operators, which form the basis of further convergence arguments. Then we proceed to a discussion of several state-of-the art algorithms and their (theoretical) convergence properties. After a section discussing issues related to the use of analogous iterative schemes for ill-posed problems, we present some practical convergence studies in numerical examples related to PET and spectral CT reconstruction.

\footnotetext{
*University of Münster, Department of Mathematics and Computer Science, 48149 Münster, Germany

${ }^{\dagger}$ University of Mannheim, Dept. of Mathematics and Computer Science, A5, 68131 Mannheim, Germany
} 


\section{Notation}

In the following we summarize the notations and definitions that will be used throughout the presented chapter:

- $x_{+}:=\max \{x, 0\}, x \in \mathbb{R}^{d}$, whereby the maximum operation has to be interpreted componentwise.

- $\iota_{C}$ is the indicator function of a set $C \subseteq \mathbb{R}^{d}$ given by

$$
\iota_{C}(x):=\left\{\begin{aligned}
0 & \text { if } x \in C \\
+\infty & \text { otherwise. }
\end{aligned}\right.
$$

- $\Gamma_{0}\left(\mathbb{R}^{d}\right)$ is a set of proper, convex, and lower semi-continuous functions mapping from $\mathbb{R}^{d}$ into the extended real numbers $\mathbb{R} \cup\{+\infty\}$.

- $\operatorname{dom} f:=\left\{x \in \mathbb{R}^{d}: f(x)<+\infty\right\}$ denotes the effective domain of $f$.

- $\partial f\left(x_{0}\right):=\left\{p \in \mathbb{R}^{d}: f(x)-f\left(x_{0}\right) \geq\left\langle p, x-x_{0}\right\rangle \forall x \in \mathbb{R}^{d}\right\}$ denotes the subdifferential of $f \in \Gamma_{0}\left(\mathbb{R}^{d}\right)$ at $x_{0} \in \operatorname{dom} f$ and is the set consisting of the subgradients of $f$ at $x_{0}$. If $f \in \Gamma_{0}\left(\mathbb{R}^{d}\right)$ is differentiable at $x_{0}$, then $\partial f\left(x_{0}\right)=\left\{\nabla f\left(x_{0}\right)\right\}$. Conversely, if $\partial f\left(x_{0}\right)$ contains only one element then $f$ is differentiable at $x_{0}$ and this element is just the gradient of $f$ at $x_{0}$. By Fermat's rule, $\hat{x}$ is a global minimizer of $f \in \Gamma_{0}\left(\mathbb{R}^{d}\right)$ if and only if

$$
0 \in \partial f(\hat{x}) .
$$

- $f^{*}(p):=\sup _{x \in \mathbb{R}^{d}}\{\langle p, x\rangle-f(x)\}$ is the (Fenchel) conjugate of $f$. For proper $f$, we have $f^{*}=f$ if and only if $f(x)=\frac{1}{2}\|x\|_{2}^{2}$. If $f \in \Gamma_{0}\left(\mathbb{R}^{d}\right)$ is positively homogeneous, i.e., $f(\alpha x)=\alpha f(x)$ for all $\alpha>0$, then

$$
f^{*}\left(x^{*}\right)=\iota_{C_{f}}\left(x^{*}\right), \quad C_{f}:=\left\{x^{*} \in \mathbb{R}^{d}:\left\langle x^{*}, x\right\rangle \leq f(x) \forall x \in \mathbb{R}^{d}\right\} .
$$

In particular, the conjugate functions of $\ell_{p}$-norms, $p \in[1,+\infty]$, are given by

$$
\|\cdot\|_{p}^{*}\left(x^{*}\right)=\iota_{B_{q}(1)}\left(x^{*}\right)
$$

where $\frac{1}{p}+\frac{1}{q}=1$ and as usual $p=1$ corresponds to $q=\infty$ and conversely, and $B_{q}(\lambda):=\left\{x \in \mathbb{R}^{d}:\|x\|_{q} \leq \lambda\right\}$ denotes the ball of radius $\lambda>0$ with respect to the $\ell_{q}$-norm.

\section{Proximal Operator}

The algorithms proposed in this chapter to solve various problems in digital image analysis and restoration have in common that they basically reduce to the evaluation of a series of proximal problems. Therefore we start with these kind of problems. For a comprehensive overview on proximal algorithms we refer to [132]. 


\subsection{Definition and Basic Properties}

For $f \in \Gamma_{0}\left(\mathbb{R}^{d}\right)$ and $\lambda>0$, the proximal operator $\operatorname{prox}_{\lambda f}: \mathbb{R}^{d} \rightarrow \mathbb{R}^{d}$ of $\lambda f$ is defined by

$$
\operatorname{prox}_{\lambda f}(x):=\underset{y \in \mathbb{R}^{d}}{\operatorname{argmin}}\left\{\frac{1}{2 \lambda}\|x-y\|_{2}^{2}+f(y)\right\} .
$$

It compromises between minimizing $f$ and being near to $x$, where $\lambda$ is the trade-off parameter between these terms. The Moreau envelope or Moreau-Yoshida regularization ${ }^{\lambda} f: \mathbb{R}^{d} \rightarrow \mathbb{R}$ is given by

$$
\lambda^{\lambda} f(x):=\min _{y \in \mathbb{R}^{d}}\left\{\frac{1}{2 \lambda}\|x-y\|_{2}^{2}+f(y)\right\} .
$$

A straightforward calculation shows that ${ }^{\lambda} f=\left(f^{*}+\frac{1}{2}\|\cdot\|_{2}^{2}\right)^{*}$. The following theorem ensures that the minimizer in (2) exists, is unique and can be characterized by a variational inequality. The Moreau envelope can be considered as a smooth approximation of $f$. For the proof we refer to 8$]$.

Theorem 3.1. Let $f \in \Gamma_{0}\left(\mathbb{R}^{d}\right)$. Then,

i) For any $x \in \mathbb{R}^{d}$, there exists a unique minimizer $\hat{x}=\operatorname{prox}_{\lambda f}(x)$ of (2).

ii) The variational inequality

$$
\frac{1}{\lambda}\langle x-\hat{x}, y-\hat{x}\rangle+f(\hat{x})-f(y) \leq 0 \quad \forall y \in \mathbb{R}^{d} .
$$

is necessary and sufficient for $\hat{x}$ to be the minimizer of (2).

iii) $\hat{x}$ is a minimizer of $f$ if and only if it is a fixed point of $\operatorname{prox}_{\lambda f}$, i.e.,

$$
\hat{x}=\operatorname{prox}_{\lambda f}(\hat{x}) .
$$

iv) The Moreau envelope ${ }^{\lambda} f$ is continuously differentiable with gradient

$$
\nabla\left({ }^{\lambda} f\right)(x)=\frac{1}{\lambda}\left(x-\operatorname{prox}_{\lambda f}(x)\right) .
$$

$\mathrm{v}$ The set of minimizers of $f$ and ${ }^{\lambda} f$ are the same.

Rewriting iv) as $\operatorname{prox}_{\lambda f}(x)=x-\lambda \nabla\left({ }^{\lambda} f\right)(x)$ we can interpret $\operatorname{prox}_{\lambda f}(x)$ as a gradient descent step with step size $\lambda$ for minimizing ${ }^{\lambda} f$.

Example 3.2. Consider the univariate function $f(y):=|y|$ and

$$
\operatorname{prox}_{\lambda f}(x)=\underset{y \in \mathbb{R}}{\operatorname{argmin}}\left\{\frac{1}{2 \lambda}(x-y)^{2}+|y|\right\} .
$$

Then, a straightforward computation yields that $\operatorname{prox}_{\lambda f}$ is the soft-shrinkage function $S_{\lambda}$ with threshold $\lambda$ (see Fig. 1) defined by

$$
S_{\lambda}(x):=(x-\lambda)_{+}-(-x-\lambda)_{+}=\left\{\begin{array}{cl}
x-\lambda & \text { for } x>\lambda, \\
0 & \text { for } x \in[-\lambda, \lambda] \\
x+\lambda & \text { for } x<-\lambda .
\end{array}\right.
$$


Setting $\hat{x}:=S_{\lambda}(x)=\operatorname{prox}_{\lambda f}(x)$, we get

$$
{ }^{\prime} f(x)=|\hat{x}|+\frac{1}{2 \lambda}(x-\hat{x})^{2}=\left\{\begin{array}{cl}
x-\frac{\lambda}{2} & \text { for } x>\lambda, \\
\frac{1}{2 \lambda} x^{2} & \text { for } x \in[-\lambda, \lambda], \\
-x-\frac{\lambda}{2} & \text { for } x<-\lambda .
\end{array}\right.
$$

This function ${ }^{\lambda} f$ is known as Huber function (see Fig. 1).
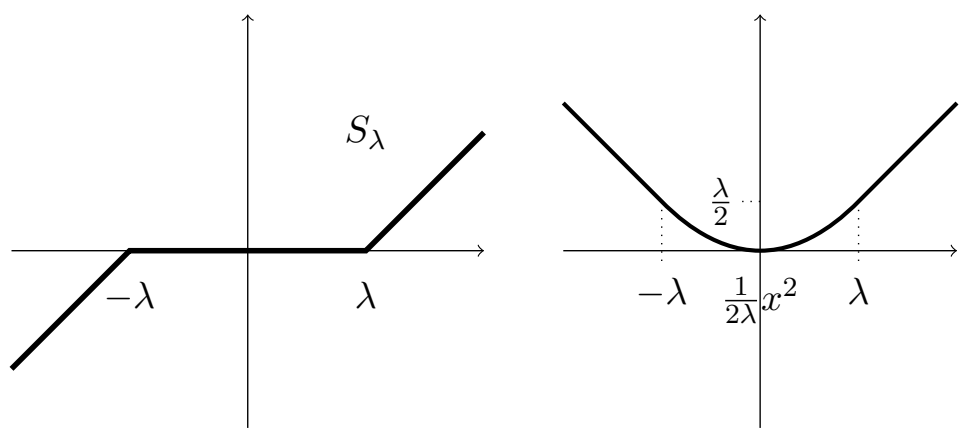

Figure 1: Left: Soft-shrinkage function $\operatorname{prox}_{\lambda f}=S_{\lambda}$ for $f(y)=|y|$. Right: Moreau envelope ${ }^{\lambda} f$.

Theorem 3.3 (Moreau decomposition). For $f \in \Gamma_{0}\left(\mathbb{R}^{d}\right)$ the following decomposition holds:

$$
\begin{gathered}
\operatorname{prox}_{f}(x)+\operatorname{prox}_{f^{*}}(x)=x, \\
{ }^{1} f(x)+{ }^{1} f^{*}(x)=\frac{1}{2}\|x\|_{2}^{2} .
\end{gathered}
$$

For a proof we refer to [141, Theorem 31.5].

Remark 3.4 (Proximal operator and resolvent). The subdifferential operator is a set-valued function $\partial f: \mathbb{R}^{d} \rightarrow 2^{\mathbb{R}^{d}}$. For $f \in \Gamma_{0}\left(\mathbb{R}^{d}\right)$, we have by Fermat's rule and subdifferential calculus that $\hat{x}=\operatorname{prox}_{\lambda \partial f}(x)$ if and only if

$$
\begin{aligned}
& 0 \in \hat{x}-x+\lambda \partial f(\hat{x}), \\
& x \in(I+\lambda \partial f)(\hat{x}),
\end{aligned}
$$

which implies by the uniqueness of the proximum that $\hat{x}=(I+\lambda \partial f)^{-1}(x)$. In particular, $J_{\lambda \partial f}:=(I+\lambda \partial f)^{-1}$ is a single-valued operator which is called the resolvent of the set-valued operator $\lambda \partial f$. In summary, the proximal operator of $\lambda f$ coincides with the resolvent of $\lambda \partial f$, i.e.,

$$
\operatorname{prox}_{\lambda f}=J_{\lambda \partial f} .
$$

The proximal operator (2) and the proximal algorithms described in Section 5 can be generalized by introducing a symmetric, positive definite matrix $Q \in \mathbb{R}^{d, d}$ as follows:

$$
\operatorname{prox}_{Q, \lambda f}:=\underset{y \in \mathbb{R}^{d}}{\operatorname{argmin}}\left\{\frac{1}{2 \lambda}\|x-y\|_{Q}^{2}+f(y)\right\},
$$

where $\|x\|_{Q}^{2}:=x^{\mathrm{T}} Q x$, see, e.g., [49, [54, 181]. 


\subsection{Special Proximal Operators}

Algorithms involving the solution of proximal problems are only efficient if the corresponding proximal operators can be evaluated in an efficient way. In the following we collect frequently appearing proximal mappings in image processing. For epigraphical projections see [12, 47, 88.

\subsubsection{Orthogonal Projections}

The proximal operator generalizes the orthogonal projection operator. The orthogonal projection of $x \in \mathbb{R}^{d}$ onto a non-empty, closed, convex set $\mathrm{C}$ is given by

$$
\Pi_{C}(x):=\underset{y \in C}{\operatorname{argmin}}\|x-y\|_{2}
$$

and can be rewritten for any $\lambda>0$ as

$$
\Pi_{C}(x)=\underset{y \in \mathbb{R}^{d}}{\operatorname{argmin}}\left\{\frac{1}{2 \lambda}\|x-y\|_{2}^{2}+\iota_{C}(y)\right\}=\operatorname{prox}_{\lambda_{\iota_{C}}}(x) .
$$

Some special sets $C$ are considered next.

Affine set $C:=\left\{y \in \mathbb{R}^{d}: A y=b\right\}$ with $A \in \mathbb{R}^{m, d}, b \in \mathbb{R}^{m}$.

In case of $\|x-y\|_{2} \rightarrow \min _{y}$ subject to $A y=b$ we substitute $z:=x-y$ which leads to

$$
\|z\|_{2} \rightarrow \min _{z} \quad \text { subject to } \quad A z=r:=A x-b .
$$

This can be directly solved, see [20], and leads after back-substitution to

$$
\Pi_{C}(x)=x-A^{\dagger}(A x-b)
$$

where $A^{\dagger}$ denotes the Moore-Penrose inverse of $A$.

Halfspace $C:=\left\{y \in \mathbb{R}^{d}: a^{\mathrm{T}} y \leq b\right\}$ with $a \in \mathbb{R}^{d}, b \in \mathbb{R}$.

A straightforward computation gives

$$
\Pi_{C}(x)=x-\frac{\left(a^{\mathrm{T}} x-b\right)_{+}}{\|a\|_{2}^{2}} a .
$$

Box and Nonnegative Orthant $C:=\left\{y \in \mathbb{R}^{d}: l \leq y \leq u\right\}$ with $l, u \in \mathbb{R}^{d}$.

The proximal operator can be applied componentwise and gives

$$
\left(\Pi_{C}(x)\right)_{k}= \begin{cases}l_{k} & \text { if } x_{k}<l_{k}, \\ x_{k} & \text { if } l_{k} \leq x_{k} \leq u_{k}, \\ u_{k} & \text { if } x_{k}>u_{k}\end{cases}
$$

For $l=0$ and $u=+\infty$ we get the orthogonal projection onto the non-negative orthant

$$
\Pi_{C}(x)=x_{+} .
$$


Probability Simplex $C:=\left\{y \in \mathbb{R}^{d}: \mathbf{1}^{\mathrm{T}} y=\sum_{k=1}^{d} y_{k}=1, y \geq 0\right\}$.

Here we have

$$
\Pi_{C}(x)=(x-\mu \mathbf{1})_{+},
$$

where $\mu \in \mathbb{R}$ has to be determined such that $h(\mu):=\mathbf{1}^{\mathrm{T}}(x-\mu \mathbf{1})_{+}=1$. Now $\mu$ can be found, e.g., by bisection with starting interval $\left[\max _{k} x_{k}-1, \max _{k} x_{k}\right]$ or by a method similar to those described in Subsection 3.2 .2 for projections onto the $\ell_{1}$-ball. Note that $h$ is a linear spline function with knots $x_{1}, \ldots, x_{d}$ so that $\mu$ is completely determined if we know the neighbor values $x_{k}$ of $\mu$.

\subsubsection{Vector Norms}

We consider the proximal operator of $f=\|\cdot\|_{p}, p \in[1,+\infty]$. By the Moreau decomposition in Theorem 3.3, regarding $(\lambda f)^{*}=\lambda f^{*}(\cdot / \lambda)$ and by (1) we obtain

$$
\begin{aligned}
\operatorname{prox}_{\lambda f}(x) & =x-\operatorname{prox}_{\lambda f^{*}}(\dot{\bar{\lambda}}) \\
& =x-\Pi_{B_{q}(\lambda)}(x),
\end{aligned}
$$

where $\frac{1}{p}+\frac{1}{q}=1$. Thus the proximal operator can be simply computed by the projections onto the $\ell_{q}$-ball. In particular, it follows for $p=1,2, \infty$ :

$p=1, q=\infty:$ For $k=1, \ldots, d$,

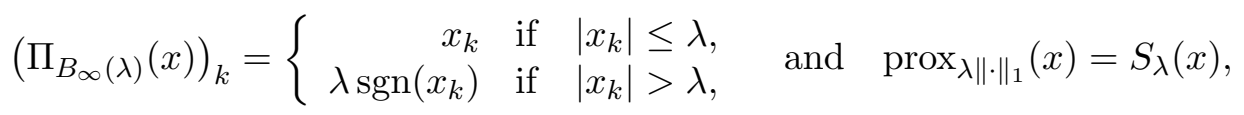

where $S_{\lambda}(x), x \in \mathbb{R}^{d}$, denotes the componentwise soft-shrinkage with threshold $\lambda$.

$p=q=2:$

$$
\Pi_{B_{2, \lambda}}(x)=\left\{\begin{array}{ccc}
x & \text { if } & \|x\|_{2} \leq \lambda, \\
\lambda \frac{x}{\|x\|_{2}} & \text { if } & \|x\|_{2}>\lambda,
\end{array} \quad \text { and } \quad \operatorname{prox}_{\lambda\left\|_{1}\right\|_{2}}(x)=\left\{\begin{array}{ccc}
0 & \text { if } \quad\|x\|_{2} \leq \lambda, \\
x\left(1-\frac{\lambda}{\|x\|_{2}}\right) & \text { if } \quad\|x\|_{2}>\lambda
\end{array}\right.\right.
$$

$p=\infty, q=1:$

$$
\Pi_{B_{1, \lambda}}(x)=\left\{\begin{array}{ccc}
x & \text { if } & \|x\|_{1} \leq \lambda \\
S_{\mu}(x) & \text { if } & \|x\|_{1}>\lambda
\end{array}\right.
$$

and

$$
\operatorname{prox}_{\lambda\|\cdot\|_{\infty}}(x)=\left\{\begin{array}{ccc}
0 & \text { if } & \|x\|_{1} \leq \lambda \\
x-S_{\mu}(x) & \text { if } & \|x\|_{1}>\lambda
\end{array}\right.
$$

with $\mu:=\frac{\left|x_{\pi(1)}\right|+\ldots+\left|x_{\pi(m)}\right|-\lambda}{m}$, where $\left|x_{\pi(1)}\right| \geq \ldots \geq\left|x_{\pi(d)}\right| \geq 0$ are the sorted absolute values of the components of $x$ and $m \leq d$ is the largest index such that $\left|x_{\pi(m)}\right|$ is positive and $\frac{\left|x_{\pi(1)}\right|+\ldots+\left|x_{\pi(m)}\right|-\lambda}{m} \leq\left|x_{\pi(m)}\right|$, see also [59, 62]. Another method follows similar lines as the projection onto the probability simplex in the previous subsection. 
Further, grouped/mixed $\ell_{2}-\ell_{p}$-norms are defined for $x=\left(x_{1}, \ldots, x_{n}\right)^{\mathrm{T}} \in \mathbb{R}^{d n}$ with $x_{j}:=$ $\left(x_{j k}\right)_{k=1}^{d} \in \mathbb{R}^{d}, j=1, \ldots, n$ by

$$
\|x\|_{2, p}:=\left\|\left(\left\|x_{j}\right\|_{2}\right)_{j=1}^{n}\right\|_{p} .
$$

For the $\ell_{2}-\ell_{1}$-norm we see that

$$
\operatorname{prox}_{\lambda\|\cdot\|_{2,1}}(x)=\underset{y \in \mathbb{R}^{d n}}{\operatorname{argmin}}\left\{\frac{1}{2 \lambda}\|x-y\|_{2}^{2}+\|y\|_{2,1}\right\}
$$

can be computed separately for each $j$ which results by the above considerations for the $\ell_{2}$-norm for each $j$ in

$$
\operatorname{prox}_{\lambda\|\cdot\|_{2}}\left(x_{j}\right)=\left\{\begin{array}{ccc}
0 & \text { if } & \left\|x_{j}\right\|_{2} \leq \lambda, \\
x_{j}\left(1-\frac{\lambda}{\left\|x_{j}\right\|_{2}}\right) & \text { if } & \left\|x_{j}\right\|_{2}>\lambda .
\end{array}\right.
$$

The procedure for evaluating $\operatorname{prox}_{\lambda\|\cdot\|_{2,1}}$ is sometimes called coupled or grouped shrinkage.

Finally, we provide the following rule from [53, Prop. 3.6].

Lemma 3.5. Let $f=g+\mu|\cdot|$, where $g \in \Gamma_{0}(\mathbb{R})$ is differentiable at 0 with $g^{\prime}(0)=0$. Then $\operatorname{prox}_{\lambda f}=\operatorname{prox}_{\lambda g} \circ S_{\lambda \mu}$.

Example 3.6. Consider the elastic net regularizer $f(x):=\frac{1}{2}\|x\|_{2}^{2}+\mu\|x\|_{1}$, see [183]. Setting the gradient in the proximal operator of $g:=\frac{1}{2}\|\cdot\|_{2}^{2}$ to zero we obtain

$$
\operatorname{prox}_{\lambda g}(x)=\frac{1}{1+\lambda} x .
$$

The whole proximal operator of $f$ can be then evaluated componentwise and we see by Lemma 3.5 that

$$
\operatorname{prox}_{\lambda f}(x)=\operatorname{prox}_{\lambda g}\left(S_{\lambda \mu}(x)\right)=\frac{1}{1+\lambda} S_{\mu \lambda}(x) .
$$

\subsubsection{Matrix Norms}

Next we deal with proximation problems involving matrix norms. For $X \in \mathbb{R}^{m, n}$, we are looking for

$$
\operatorname{prox}_{\lambda\|\cdot\|}(X)=\underset{Y \in \mathbb{R}^{m, n}}{\operatorname{argmin}}\left\{\frac{1}{2 \lambda}\|X-Y\|_{\mathcal{F}}^{2}+\|Y\|\right\},
$$

where $\|\cdot\|_{\mathcal{F}}$ is the Frobenius norm and $\|\cdot\|$ is any unitarily invariant matrix norm, i.e., $\|X\|=\left\|U X V^{T}\right\|$ for all unitary matrices $U \in \mathbb{R}^{m, m}, V \in \mathbb{R}^{n, n}$. Von Neumann (1937) [169] has characterized the unitarily invariant matrix norms as those matrix norms which can be written in the form

$$
\|X\|=g(\sigma(X)),
$$

where $\sigma(X)$ is the vector of singular values of $X$ and $g$ is a symmetric gauge function, see [175]. Recall that $g: \mathbb{R}^{d} \rightarrow \mathbb{R}_{+}$is a symmetric gauge function if it is a positively homogeneous convex function which vanishes at the origin and fulfills

$$
g(x)=g\left(\epsilon_{1} x_{k_{1}}, \ldots, \epsilon_{k} x_{k_{d}}\right)
$$


for all $\epsilon_{k} \in\{-1,1\}$ and all permutations $k_{1}, \ldots, k_{d}$ of indices. An analogous result was given by Davis [60] for symmetric matrices, where $V^{\mathrm{T}}$ is replaced by $U^{\mathrm{T}}$ and the singular values by the eigenvalues.

We are interested in the Schatten- $p$ norms for $p=1,2, \infty$ which are defined for $X \in \mathbb{R}^{m, n}$ and $t:=\min \{m, n\}$ by

$$
\begin{aligned}
\|X\|_{*} & :=\sum_{i=1}^{t} \sigma_{i}(X)=g_{*}(\sigma(X))=\|\sigma(X)\|_{1}, \quad \text { (Nuclear norm) } \\
\|X\|_{\mathcal{F}} & :=\left(\sum_{i=1}^{m} \sum_{j=1}^{n} x_{i j}^{2}\right)^{\frac{1}{2}}=\left(\sum_{i=1}^{t} \sigma_{i}(X)^{2}\right)^{\frac{1}{2}}=g_{\mathcal{F}}(\sigma(X))=\|\sigma(X)\|_{2}, \quad \text { (Frobenius norm) } \\
\|X\|_{2} & :=\max _{i=1, \ldots, t} \sigma_{i}(X)=g_{2}(\sigma(X))=\|\sigma(X)\|_{\infty}, \quad \text { (Spectral norm). }
\end{aligned}
$$

The following theorem shows that the solution of (6) reduces to a proximal problem for the vector norm of the singular values of $X$. Another proof for the special case of the nuclear norm can be found in [37].

Theorem 3.7. Let $X=U \Sigma_{X} V^{T}$ be the singular value decomposition of $X$ and $\|\cdot\|$ a unitarily invariant matrix norm. Then $\operatorname{prox}_{\lambda\|\cdot\|}(X)$ in (6) is given by $\hat{X}=U \Sigma_{\hat{X}} V^{T}$, where the singular values $\sigma(\hat{X})$ in $\Sigma_{\hat{X}}$ are determined by

$$
\sigma(\hat{X}):=\operatorname{prox}_{\lambda g}(\sigma(X))=\underset{\sigma \in \mathbb{R}^{t}}{\operatorname{argmin}}\left\{\frac{1}{2}\|\sigma(X)-\sigma\|_{2}^{2}+\lambda g(\sigma)\right\}
$$

with the symmetric gauge function $g$ corresponding to $\|\cdot\|$.

Proof. By Fermat's rule we know that the solution $\hat{X}$ of $(6)$ is determined by

$$
0 \in \hat{X}-X+\lambda \partial\|\hat{X}\|
$$

and from [175] that

$$
\partial\|X\|=\operatorname{conv}\left\{U D V^{\mathrm{T}}: X=U \Sigma_{X} V^{\mathrm{T}}, D=\operatorname{diag}(d), d \in \partial g(\sigma(X))\right\} .
$$

We now construct the unique solution $\hat{X}$ of (8). Let $\hat{\sigma}$ be the unique solution of (7). By Fermat's rule $\hat{\sigma}$ satisfies $0 \in \hat{\sigma}-\sigma(X)+\lambda \partial g(\hat{\sigma})$ and consequently there exists $d \in \partial g(\hat{\sigma})$ such that

$$
0=U\left(\operatorname{diag}(\hat{\sigma})-\Sigma_{X}+\lambda \operatorname{diag}(d)\right) V_{F}^{\mathrm{T}} \Leftrightarrow 0=U \operatorname{diag}(\hat{\sigma}) V^{\mathrm{T}}-X+\lambda U \operatorname{diag}(d) V^{\mathrm{T}} .
$$

By (9) we see that $\hat{X}:=U \operatorname{diag}(\hat{\sigma}) V^{\mathrm{T}}$ is a solution of (8). This completes the proof.

For the special matrix norms considered above, we obtain by the previous subsection

$$
\begin{aligned}
\|\cdot\|_{*}: & \sigma(\hat{X}):=\sigma(X)-\Pi_{B_{\infty, \lambda}}(\sigma(X)), \\
\|\cdot\|_{\mathcal{F}}: & \sigma(\hat{X}):=\sigma(X)-\Pi_{B_{2, \lambda}}(\sigma(X)), \\
\|\cdot\|_{2}: & \sigma(\hat{X}):=\sigma(X)-\Pi_{B_{1, \lambda}}(\sigma(X)) .
\end{aligned}
$$




\section{Fixed Point Algorithms and Averaged Operators}

An operator $T: \mathbb{R}^{d} \rightarrow \mathbb{R}^{d}$ is contractive if it is Lipschitz continuous with Lipschitz constant $L<1$, i.e., there exists a norm $\|\cdot\|$ on $\mathbb{R}^{d}$ such that

$$
\|T x-T y\| \leq L\|x-y\| \quad \forall x, y \in \mathbb{R}^{d} .
$$

In case $L=1$, the operator is called nonexpansive. A function $T: \mathbb{R}^{d} \supset \Omega \rightarrow \mathbb{R}^{d}$ is firmly nonexpansive if it fulfills for all $x, y \in \mathbb{R}^{d}$ one of the following equivalent conditions [12]:

$$
\begin{aligned}
& \|T x-T y\|_{2}^{2} \leq\langle x-y, T x-T y\rangle, \\
& \|T x-T y\|_{2}^{2} \leq\|x-y\|_{2}^{2}-\|(I-T) x-(I-T) y\|_{2}^{2} .
\end{aligned}
$$

In particular we see that a firmly nonexpansive function is nonexpansive.

Lemma 4.1. For $f \in \Gamma_{0}\left(\mathbb{R}^{d}\right)$, the proximal operator $\operatorname{prox}_{\lambda f}$ is firmly nonexpansive. In particular the orthogonal projection onto convex sets is firmly nonexpansive.

Proof. By Theorem 3.1 ii) we have that

$$
\left\langle x-\operatorname{prox}_{\lambda f}(x), z-\operatorname{prox}_{\lambda f}(x)\right\rangle \leq 0 \quad \forall z \in \mathbb{R}^{d} .
$$

With $z:=\operatorname{prox}_{\lambda f}(y)$ this gives

$$
\left\langle x-\operatorname{prox}_{\lambda f}(x), \operatorname{prox}_{\lambda f}(y)-\operatorname{prox}_{\lambda f}(x)\right\rangle \leq 0
$$

and similarly

$$
\left\langle y-\operatorname{prox}_{\lambda f}(y), \operatorname{prox}_{\lambda f}(x)-\operatorname{prox}_{\lambda f}(y)\right\rangle \leq 0 .
$$

Adding these inequalities we obtain

$$
\begin{aligned}
&\left\langle x-\operatorname{prox}_{\lambda f}(x)+\operatorname{prox}_{\lambda f}(y)-y, \operatorname{prox}_{\lambda f}(y)-\operatorname{prox}_{\lambda f}(x)\right\rangle \leq 0 \\
&\left\|\operatorname{prox}_{\lambda f}(y)-\operatorname{prox}_{\lambda f}(x)\right\|_{2}^{2} \leq\left\langle y-x, \operatorname{prox}_{\lambda f}(y)-\operatorname{prox}_{\lambda f}(x)\right\rangle .
\end{aligned}
$$

The Banach fixed point theorem guarantees that a contraction has a unique fixed point and that the Picard sequence

$$
x^{(r+1)}=T x^{(r)}
$$

converges to this fixed point for every initial element $x^{(0)}$. However, in many applications the contraction property is too restrictive in the sense that we often do not have a unique fixed point. Indeed, it is quite natural in many cases that the reached fixed point depends on the starting value $x^{(0)}$. Note that if $T$ is continuous and $\left(T^{r} x^{(0)}\right)_{r \in \mathbb{N}}$ is convergent, then it converges to a fixed point of $T$. In the following, we denote by $\operatorname{Fix}(T)$ the set of fixed points of $T$. Unfortunately, we do not have convergence of $\left(T^{r} x^{(0)}\right)_{r \in \mathbb{N}}$ just for nonexpansive operators as the following example shows. 
Example 4.2. In $\mathbb{R}^{2}$ we consider the reflection operator

$$
R:=\left(\begin{array}{rr}
1 & 0 \\
0 & -1
\end{array}\right)
$$

Obviously, $R$ is nonexpansive and we only have convergence of $\left(R^{r} x^{(0)}\right)_{r \in \mathbb{N}}$ if $x^{(0)} \in \operatorname{Fix}(R)=$ $\operatorname{span}\left\{(1,0)^{\mathrm{T}}\right\}$. A possibility to obtain a 'better' operator is to average $R$, i.e., to build

$$
T:=\alpha I+(1-\alpha) R=\left(\begin{array}{rr}
1 & 0 \\
0 & 2 \alpha-1
\end{array}\right), \quad \alpha \in(0,1) .
$$

By

$$
T x=x \Leftrightarrow \alpha x+(1-\alpha) R(x)=x \Leftrightarrow(1-\alpha) R(x)=(1-\alpha) x,
$$

we see that $R$ and $T$ have the same fixed points. Moreover, since $2 \alpha-1 \in(-1,1)$, the sequence $\left(T^{r} x^{(0)}\right)_{r \in \mathbb{N}}$ converges to $\left(x_{1}^{(0)}, 0\right)^{\mathrm{T}}$ for every $x^{(0)}=\left(x_{1}^{(0)}, x_{2}^{(0)}\right)^{\mathrm{T}} \in \mathbb{R}^{2}$.

An operator $T: \mathbb{R}^{d} \rightarrow \mathbb{R}^{d}$ is called averaged if there exists a nonexpansive mapping $R$ and a constant $\alpha \in(0,1)$ such that

$$
T=\alpha I+(1-\alpha) R
$$

Following 12 we see that

$$
\operatorname{Fix}(R)=\operatorname{Fix}(T) .
$$

Historically, the concept of averaged mappings can be traced back to [106, 113, 149], where the name 'averaged' was not used yet. Results on averaged operators can also be found, e.g., in [12, 36, 52].

Lemma 4.3 (Averaged, (Firmly) Nonexpansive and Contractive Operators).

i) Every averaged operator is nonexpansive.

ii) A contractive operator $T: \mathbb{R}^{d} \rightarrow \mathbb{R}^{d}$ with Lipschitz constant $L<1$ is averaged with respect to all parameters $\alpha \in(0,(1-L) / 2]$.

iii) An operator is firmly nonexpansive if and only if it is averaged with $\alpha=\frac{1}{2}$.

Proof. i) Let $T=\alpha I+(1-\alpha) R$ be averaged. Then the first assertion follows by

$$
\|T(x)-T(y)\|_{2} \leq \alpha\|x-y\|_{2}+(1-\alpha)\|R(x)-R(y)\|_{2} \leq\|x-y\|_{2} .
$$

ii) We define the operator $R:=\frac{1}{1-\alpha}(T-\alpha I)$. It holds for all $x, y \in \mathbb{R}^{d}$ that

$$
\begin{aligned}
\|R x-R y\|_{2} & =\frac{1}{1-\alpha}\|(T-\alpha I) x-(T-\alpha I) y\|_{2}, \\
& \leq \frac{1}{1-\alpha}\|T x-T y\|_{2}+\frac{\alpha}{1-\alpha}\|x-y\|_{2}, \\
& \leq \frac{L+\alpha}{1-\alpha}\|x-y\|_{2},
\end{aligned}
$$

so $R$ is nonexpansive if $\alpha \leq(1-L) / 2$.

iii) With $R:=2 T-I=T-(I-T)$ we obtain the following equalities

$$
\begin{aligned}
\|R x-R y\|_{2}^{2} & =\|T x-T y-((I-T) x-(I-T) y)\|_{2}^{2} \\
& =-\|x-y\|_{2}^{2}+2\|T x-T y\|_{2}^{2}+2\|(I-T) x-(I-T) y\|_{2}^{2}
\end{aligned}
$$


and therefore after reordering

$$
\begin{aligned}
& \|x-y\|_{2}^{2}-\|T x-T y\|_{2}^{2}-\|(I-T) x-(I-T) y\|_{2}^{2} \\
= & \|T x-T y\|_{2}^{2}+\|(I-T) x-(I-T) y\|_{2}^{2}-\|R x-R y\|_{2}^{2} \\
= & \frac{1}{2}\left(\|x-y\|_{2}^{2}+\|R x-R y\|_{2}^{2}\right)-\|R x-R y\|_{2}^{2} \\
= & \frac{1}{2}\left(\|x-y\|_{2}^{2}-\|R x-R y\|_{2}^{2}\right) .
\end{aligned}
$$

If $R$ is nonexpansive, then the last expression is $\geq 0$ and consequently $(10)$ holds true so that $T$ is firmly nonexpansive. Conversely, if $T$ fulfills 10 , then

$$
\frac{1}{2}\left(\|x-y\|_{2}^{2}-\|R x-R y\|_{2}^{2}\right) \geq 0
$$

so that $R$ is nonexpansive. This completes the proof.

By the following lemma averaged operators are closed under composition.

Lemma 4.4 (Composition of Averaged Operators).

i) Suppose that $T: \mathbb{R}^{d} \rightarrow \mathbb{R}^{d}$ is averaged with respect to $\alpha \in(0,1)$. Then, it is also averaged with respect to any other parameter $\tilde{\alpha} \in(0, \alpha]$.

ii) Let $T_{1}, T_{2}: \mathbb{R}^{d} \rightarrow \mathbb{R}^{d}$ be averaged operators. Then, $T_{2} \circ T_{1}$ is also averaged.

Proof. i) By assumption, $T=\alpha I+(1-\alpha) R$ with $R$ nonexpansive. We have

$$
T=\tilde{\alpha} I+((\alpha-\tilde{\alpha}) I+(1-\alpha) R)=\tilde{\alpha} I+(1-\tilde{\alpha}) \underbrace{\left(\frac{\alpha-\tilde{\alpha}}{1-\tilde{\alpha}} I+\frac{1-\alpha}{1-\tilde{\alpha}} R\right)}_{\tilde{R}}
$$

and for all $x, y \in \mathbb{R}^{d}$ it holds that

$$
\|\tilde{R}(x)-\tilde{R}(y)\|_{2} \leq \frac{\alpha-\tilde{\alpha}}{1-\tilde{\alpha}}\|x-y\|_{2}+\frac{1-\alpha}{1-\tilde{\alpha}}\|R(x)-R(y)\|_{2} \leq\|x-y\|_{2} .
$$

So, $\tilde{R}$ is nonexpansive.

ii) By assumption there exist nonexpansive operators $R_{1}, R_{2}$ and $\alpha_{1}, \alpha_{2} \in(0,1)$ such that

$$
\begin{aligned}
T_{2}\left(T_{1}(x)\right) & =\alpha_{2} T_{1}(x)+\left(1-\alpha_{2}\right) R_{2}\left(T_{1}(x)\right) \\
& =\alpha_{2}\left(\alpha_{1} x+\left(1-\alpha_{1}\right) R_{1}(x)\right)+\left(1-\alpha_{2}\right) R_{2}\left(T_{1}(x)\right) \\
& =\underbrace{\alpha_{2} \alpha_{1}}_{:=\alpha} x+(\alpha_{2}-\underbrace{\alpha_{2} \alpha_{1}}_{=\alpha}) R_{1}(x)+\left(1-\alpha_{2}\right) R_{2}\left(T_{1}(x)\right) \\
& =\alpha x+(1-\alpha) \underbrace{\left(\frac{\alpha_{2}-\alpha}{1-\alpha} R_{1}(x)+\frac{1-\alpha_{2}}{1-\alpha} R_{2}\left(T_{1}(x)\right)\right)}_{=: R}
\end{aligned}
$$

The concatenation of two nonexpansive operators is nonexpansive. Finally, the convex combination of two nonexpansive operators is nonexpansive so that $R$ is indeed nonexpansive. 
An operator $T: \mathbb{R}^{d} \rightarrow \mathbb{R}^{d}$ is called asymptotically regular if it holds for all $x \in \mathbb{R}^{d}$ that

$$
\left(T^{r+1} x-T^{r} x\right) \rightarrow 0 \quad \text { for } \quad r \rightarrow+\infty .
$$

Note that this property does not imply convergence, even boundedness cannot be guaranteed. As an example consider the partial sums of a harmonic sequence.

Theorem 4.5 (Asymptotic Regularity of Averaged Operators). Let $T: \mathbb{R}^{d} \rightarrow \mathbb{R}^{d}$ be an averaged operator with respect to the nonexpansive mapping $R$ and the parameter $\alpha \in(0,1)$. Assume that $\operatorname{Fix}(T) \neq \emptyset$. Then, $T$ is asymptotically regular.

Proof. Let $\hat{x} \in \operatorname{Fix}(T)$ and $x^{(r)}=T^{r} x^{(0)}$ for some starting element $x^{(0)}$. Since $T$ is nonexpansive, i.e., $\left\|x^{(r+1)}-\hat{x}\right\|_{2} \leq\left\|x^{(r)}-\hat{x}\right\|_{2}$ we obtain

$$
\lim _{r \rightarrow \infty}\left\|x^{(r)}-\hat{x}\right\|_{2}=d \geq 0 .
$$

Using $\operatorname{Fix}(T)=\operatorname{Fix}(R)$ it follows

$$
\lim _{r \rightarrow \infty} \sup \left\|R\left(x^{(r)}\right)-\hat{x}\right\|_{2}=\lim _{r \rightarrow \infty} \sup \left\|R\left(x^{(r)}\right)-R(\hat{x})\right\|_{2} \leq \lim _{r \rightarrow \infty}\left\|x^{(r)}-\hat{x}\right\|_{2}=d .
$$

Assume that $\left\|x^{(r+1)}-x^{(r)}\right\|_{2} \not \rightarrow 0$ for $r \rightarrow \infty$. Then, there exists a subsequence $\left(x^{\left(r_{l}\right)}\right)_{l \in \mathbb{N}}$ such that

$$
\left\|x^{\left(r_{l}+1\right)}-x^{\left(r_{l}\right)}\right\|_{2} \geq \varepsilon
$$

for some $\varepsilon>0$. By 13 the sequence $\left(x^{\left(r_{l}\right)}\right)_{l \in \mathbb{N}}$ is bounded. Hence there exists a convergent subsequence $\left(x^{\left(r_{l_{j}}\right)}\right)$ such that

$$
\lim _{j \rightarrow \infty} x^{\left(r_{l_{j}}\right)}=a,
$$

where $a \in S(\hat{x}, d):=\left\{x \in \mathbb{R}^{d}:\|x-\hat{x}\|_{2}=d\right\}$ by (13). On the other hand, we have by the continuity of $R$ and (14) that

$$
\lim _{j \rightarrow \infty} R\left(x^{\left(r_{l_{j}}\right)}\right)=b, \quad b \in B(\hat{x}, d) .
$$

Since $\varepsilon \leq\left\|x^{\left(r_{l_{j}}+1\right)}-x^{\left(r_{l_{j}}\right)}\right\|_{2}=\left\|(\alpha-1) x^{\left(r_{l_{j}}\right)}+(1-\alpha) R\left(x^{\left(r_{l_{j}}\right)}\right)\right\|_{2}$ we conclude by taking the limit $j \rightarrow \infty$ that $a \neq b$. By the continuity of $T$ and (13) we obtain

$$
\lim _{j \rightarrow \infty} T\left(x^{\left(r_{l_{j}}\right)}\right)=c, \quad c \in S(\hat{x}, d) .
$$

However, by the strict convexity of $\|\cdot\|_{2}^{2}$ this yields the contradiction

$$
\begin{aligned}
\|c-\hat{x}\|_{2}^{2} & =\lim _{j \rightarrow \infty}\left\|T\left(x^{\left(r_{l_{j}}\right)}\right)-\hat{x}\right\|_{2}^{2}=\lim _{j \rightarrow \infty}\left\|\alpha\left(x^{\left(r_{l_{j}}\right)}-\hat{x}\right)+(1-\alpha)\left(R\left(x^{\left(r_{l_{j}}\right)}\right)-\hat{x}\right)\right\|_{2}^{2} \\
& =\|\alpha(a-\hat{x})+(1-\alpha)(b-\hat{x})\|_{2}^{2}<\alpha\|a-\hat{x}\|_{2}^{2}+(1-\alpha)\|b-\hat{x}\|_{2}^{2} \\
& \leq d^{2}
\end{aligned}
$$

The following theorem was first proved for operators on Hilbert spaces by Opial 126, Theorem 1] based on results in [29], where convergence must be replaced by weak convergence in general Hilbert spaces. A shorter proof can be found in the appendix of [58]. For finite dimensional spaces the proof simplifies as follows. 
Theorem 4.6 (Opial's Convergence Theorem). Let $T: \mathbb{R}^{d} \rightarrow \mathbb{R}^{d}$ fulfill the following conditions: $\operatorname{Fix}(T) \neq \emptyset, T$ is nonexpansive and asymptotically regular. Then, for every $x^{(0)} \in \mathbb{R}^{d}$, the sequence of Picard iterates $\left(x^{(r)}\right)_{r \in \mathbb{N}}$ generated by $x^{(r+1)}=T x^{(r)}$ converges to an element of $\operatorname{Fix}(T)$.

Proof. Since $T$ is nonexpansive, we have for any $\hat{x} \in \operatorname{Fix}(T)$ and any $x^{(0)} \in \mathbb{R}^{d}$ that

$$
\left\|T^{r+1} x^{(0)}-\hat{x}\right\|_{2} \leq\left\|T^{r} x^{(0)}-\hat{x}\right\|_{2} .
$$

Hence $\left(T^{r} x^{(0)}\right)_{r \in \mathbb{N}}$ is bounded and there exists a subsequence $\left(T^{r_{l}} x^{(0)}\right)_{l \in \mathbb{N}}=\left(x^{\left(r_{l}\right)}\right)_{l \in \mathbb{N}}$ which converges to some $\tilde{x}$. If we can show that $\tilde{x} \in \operatorname{Fix}(T)$ we are done because in this case

$$
\left\|T^{r} x^{(0)}-\tilde{x}\right\|_{2} \leq\left\|T^{r_{l}} x^{(0)}-\tilde{x}\right\|_{2}, \quad r \geq r_{l}
$$

and thus the whole sequence converges to $\tilde{x}$.

Since $T$ is asymptotically regular it follows that

$$
(T-I)\left(T^{r_{l}} x^{(0)}\right)=T^{r_{l}+1} x^{(0)}-T^{r_{l}} x^{(0)} \rightarrow 0
$$

and since $\left(T^{r_{l}} x^{(0)}\right)_{l \in \mathbb{N}}$ converges to $\tilde{x}$ and $T$ is continuous we get that $(T-I)(\tilde{x})=0$, i.e., $\tilde{x} \in \operatorname{Fix}(T)$.

Combining the above Theorems 4.5 and 4.6 we obtain the following main result.

Theorem 4.7 (Convergence of Averaged Operator Iterations). Let $T: \mathbb{R}^{d} \rightarrow \mathbb{R}^{d}$ be an averaged operator such that $\operatorname{Fix}(T) \neq \emptyset$. Then, for every $x^{(0)} \in \mathbb{R}^{d}$, the sequence $\left(T^{r} x^{(0)}\right)_{r \in \mathbb{N}}$ converges to a fixed point of $T$.

\section{Proximal Algorithms}

\subsection{Proximal Point Algorithm}

By Theorem 3.1 iii) the minimizer of a function $f \in \Gamma_{0}\left(\mathbb{R}^{d}\right)$, which we suppose to exist, is characterized by the fixed point equation

$$
\hat{x}=\operatorname{prox}_{\lambda f}(\hat{x}) .
$$

The corresponding Picard iteration gives rise to the following proximal point algorithm which dates back to [114, 140]. Since $\operatorname{prox}_{\lambda f}$ is firmly nonexpansive by Lemma 4.1 and thus averaged, the algorithm converges by Theorem 4.7 for any initial value $x^{(0)} \in \mathbb{R}^{d}$ to a minimizer of $f$ if there exits one.

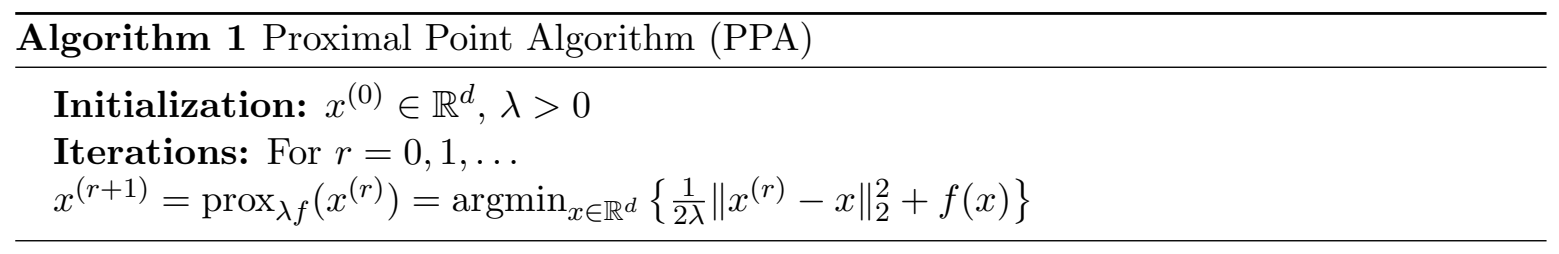

The PPA can be generalized for the sum $\sum_{i=1}^{n} f_{i}$ of functions $f_{i} \in \Gamma_{0}\left(\mathbb{R}^{d}\right), i=1, \ldots, n$. Popular generalizations are the so-called cyclic PPA [18] and the parallel PPA [50]. 


\subsection{Proximal Gradient Algorithm}

We are interested in minimizing functions of the form $f=g+h$, where $g: \mathbb{R}^{d} \rightarrow \mathbb{R}$ is convex, differentiable with Lipschitz continuous gradient and Lipschitz constant $L$, i.e.,

$$
\|\nabla g(x)-\nabla g(y)\|_{2} \leq L\|x-y\|_{2} \quad \forall x, y \in \mathbb{R}^{d},
$$

and $h \in \Gamma_{0}\left(\mathbb{R}^{d}\right)$. Note that the Lipschitz condition on $\nabla g$ implies

$$
g(x) \leq g(y)+\langle\nabla g(y), x-y\rangle+\frac{L}{2}\|x-y\|_{2}^{2} \quad \forall x, y \in \mathbb{R}^{d},
$$

see, e.g., [127]. We want to solve

$$
\underset{x \in \mathbb{R}^{d}}{\operatorname{argmin}}\{g(x)+h(x)\} .
$$

By Fermat's rule and subdifferential calculus we know that $\hat{x}$ is a minimizer of (17) if and only if

$$
\begin{aligned}
0 & \in \nabla g(\hat{x})+\partial h(\hat{x}), \\
\hat{x}-\eta \nabla g(\hat{x}) & \in \hat{x}+\eta \partial h(\hat{x}), \\
\hat{x} & =(I+\eta \partial h)^{-1}(\hat{x}-\eta \nabla g(\hat{x}))=\operatorname{prox}_{\eta h}(\hat{x}-\eta \nabla g(\hat{x})) .
\end{aligned}
$$

This is a fixed point equation for the minimizer $\hat{x}$ of $f$. The corresponding Picard iteration is known as proximal gradient algorithm or as proximal forward-backward splitting.

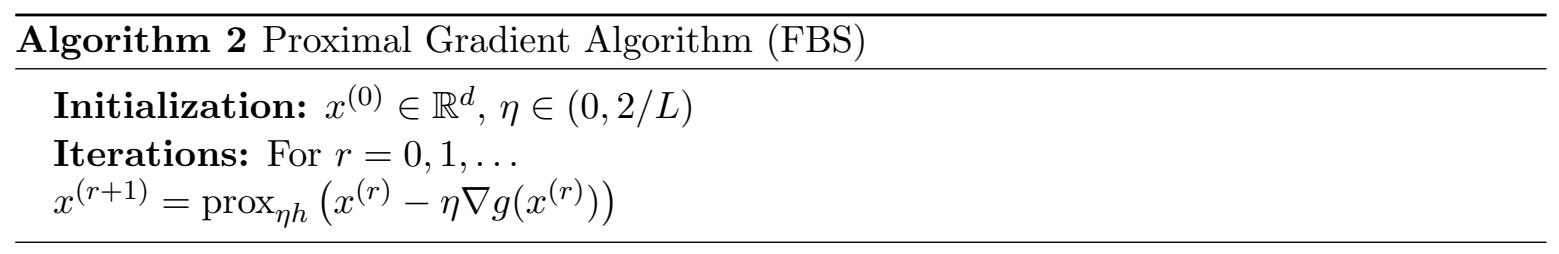

In the special case when $h:=\iota_{C}$ is the indicator function of a non-empty, closed, convex set $C \subset \mathbb{R}^{d}$, the above algorithm for finding

$$
\underset{x \in C}{\operatorname{argmin}} g(x)
$$

becomes the gradient descent re-projection algorithm.

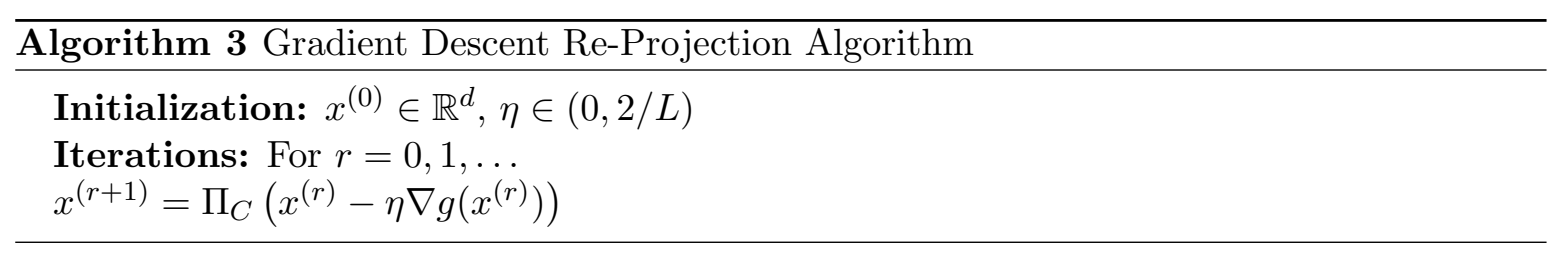

It is also possible to use flexible variables $\eta_{r} \in\left(0, \frac{2}{L}\right)$ in the proximal gradient algorithm. For further details, modifications and extensions see also [67, Chapter 12]. The convergence of the algorithm follows by the next theorem. 
Theorem 5.1 (Convergence of Proximal Gradient Algorithm). Let $g: \mathbb{R}^{d} \rightarrow \mathbb{R}$ be a convex, differentiable function on $\mathbb{R}^{d}$ with Lipschitz continuous gradient and Lipschitz constant $L$ and $h \in \Gamma_{0}\left(\mathbb{R}^{d}\right)$. Suppose that a solution of $(17)$ exists. Then, for every initial point $x^{(0)}$ and $\eta \in\left(0, \frac{2}{L}\right)$, the sequence $\left\{x^{(r)}\right\}_{r}$ generated by the proximal gradient algorithm converges to a solution of (17).

Proof. We show that $\operatorname{prox}_{\eta h}(I-\eta \nabla g)$ is averaged. Then we are done by Theorem 4.7. By Lemma 4.1 we know that prox $_{\eta h}$ is firmly nonexpansive. By the Baillon-Haddad Theorem [12, Corollary 16.1] the function $\frac{1}{L} \nabla g$ is also firmly nonexpansive, i.e., it is averaged with parameter $\frac{1}{2}$. This means that there exists a nonexpansive mapping $R$ such that $\frac{1}{L} \nabla g=$ $\frac{1}{2}(I+R)$ which implies

$$
I-\eta \nabla g=I-\frac{\eta L}{2}(I+R)=\left(1-\frac{\eta L}{2}\right) I+\frac{\eta L}{2}(-R) .
$$

Thus, for $\eta \in\left(0, \frac{2}{L}\right)$, the operator $I-\eta \nabla g$ is averaged. Since the concatenation of two averaged operators is averaged again we obtain the assertion.

Under the above conditions a linear convergence rate can be achieved in the sense that

$$
f\left(x^{(r)}\right)-f(\hat{x})=\mathcal{O}(1 / r),
$$

see, e.g., [13, 46].

Example 5.2. For solving

$$
\underset{x \in \mathbb{R}^{d}}{\operatorname{argmin}}\{\underbrace{\frac{1}{2}\|K x-b\|_{2}^{2}}_{g}+\underbrace{\lambda\|x\|_{1}}_{h}\}
$$

we compute $\nabla g(x)=K^{\mathrm{T}}(K x-b)$ and use that the proximal operator of the $\ell_{1}$-norm is just the componentwise soft-shrinkage. Then the proximal gradient algorithm becomes

$$
x^{(r+1)}=\operatorname{prox}_{\lambda \eta\|\cdot\|_{1}}\left(x^{(r)}-\eta K^{\mathrm{T}}\left(K x^{(r)}-b\right)\right)=S_{\eta \lambda}\left(x^{(r)}-\eta K^{\mathrm{T}}\left(K x^{(r)}-b\right)\right) .
$$

This algorithm is known as iterative soft-thresholding algorithm (ISTA) and was developed and analyzed through various techniques by many researchers. For a general Hilbert space approach, see, e.g., [58].

The FBS algorithm has been recently extended to the case of non-convex functions in 6 , 7. 22, 49, 125]. The convergence analysis mainly rely on the assumption that the objective function $f=g+h$ satisfies the Kurdyka-Lojasiewicz inequality which is indeed fulfilled for a wide class of functions as log - exp, semi-algebraic and subanalytic functions which are of interest in image processing.

\subsection{Accelerated Algorithms}

For large scale problems as those arising in image processing a major concern is to find efficient algorithms solving the problem in a reasonable time. While each FBS step has low 
computational complexity, it may suffer from slow linear convergence [46]. Using a simple extrapolation idea with appropriate parameters $\tau_{r}$, the convergence can often be accelerated:

$$
\begin{aligned}
y^{(r)} & =x^{(r)}+\tau_{r}\left(x^{(r)}-x^{(r-1)}\right), \\
x^{(r+1)} & =\operatorname{prox}_{\eta h}\left(y^{(r)}-\eta \nabla g\left(y^{(r)}\right)\right) .
\end{aligned}
$$

By the next Theorem 5.3 we will see that $\tau_{r}=\frac{r-1}{r+2}$ appears to be a good choice. Clearly, we can vary $\eta$ in each step again. Choosing $\theta_{r}$ such that $\tau_{r}=\frac{\theta_{r}\left(1-\theta_{r-1}\right)}{\theta_{r-1}}$, e.g., $\theta_{r}=\frac{2}{r+2}$ for the above choice of $\tau_{r}$, the algorithm can be rewritten as follows:

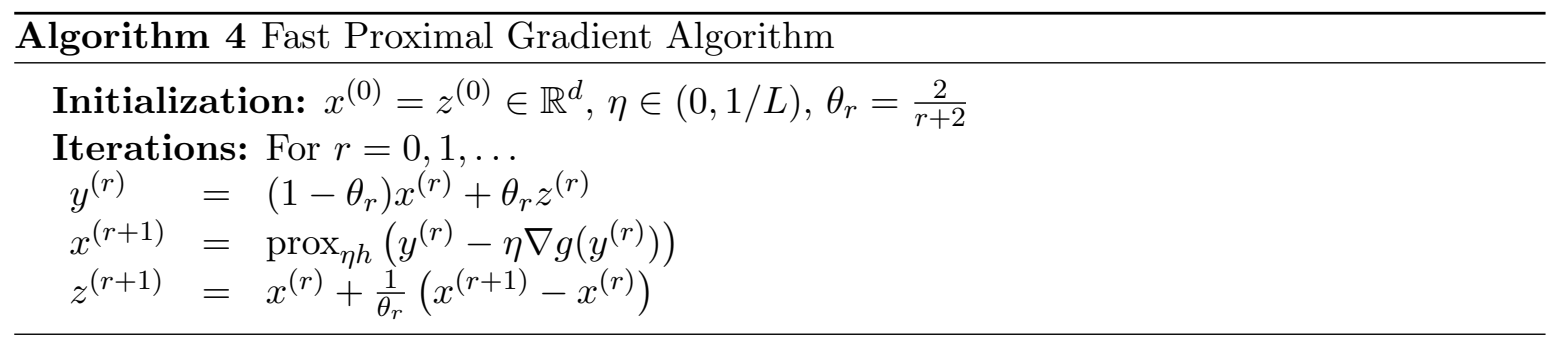

By the following standard theorem the extrapolation modification of the FBS algorithm ensures a quadratic convergence rate see also Nemirovsky and Yudin [118.

Theorem 5.3. Let $f=g+h$, where $g: \mathbb{R}^{d} \rightarrow \mathbb{R}$ is a convex, Lipschitz differentiable function with Lipschitz constant $L$ and $h \in \Gamma_{0}\left(\mathbb{R}^{d}\right)$. Assume that $f$ has a minimizer $\hat{x}$. Then the fast proximal gradient algorithm fulfills

$$
f\left(x^{(r)}\right)-f(\hat{x})=\mathcal{O}\left(1 / r^{2}\right) .
$$

Proof. First we consider the progress in one step of the algorithm. By the Lipschitz differentiability of $g$ in 16 and since $\eta<\frac{1}{L}$ we know that

$$
g\left(x^{(r+1)}\right) \leq g\left(y^{(r)}\right)+\left\langle\nabla g\left(y^{(r)}\right), x^{(r+1)}-y^{(r)}\right\rangle+\frac{1}{2 \eta}\left\|x^{(r+1)}-y^{(r)}\right\|_{2}^{2}
$$

and by the variational characterization of the proximal operator in Theorem 3.1 i) for all $u \in \mathbb{R}^{d}$ that

$$
\begin{aligned}
h\left(x^{(r+1)}\right) & \leq h(u)+\frac{1}{\eta}\left\langle y^{(r)}-\eta \nabla g\left(y^{(r)}\right)-x^{(r+1)}, x^{(r+1)}-u\right\rangle \\
& \leq h(u)-\left\langle\nabla g\left(y^{(r)}\right), x^{(r+1)}-u\right\rangle+\frac{1}{\eta}\left\langle y^{(r)}-x^{(r+1)}, x^{(r+1)}-u\right\rangle .
\end{aligned}
$$

Adding the main inequalities 200 and (21) and using the convexity of $g$ yields

$$
\begin{aligned}
f\left(x^{(r+1)}\right) & \leq f(u) \underbrace{-g(u)+g\left(y^{(r)}\right)+\left\langle\nabla g\left(y^{(r)}\right), u-y^{(r)}\right\rangle}_{\leq 0} \\
& +\frac{1}{2 \eta}\left\|x^{(r+1)}-y^{(r)}\right\|_{2}^{2}+\frac{1}{\eta}\left\langle y^{(r)}-x^{(r+1)}, x^{(r+1)}-u\right\rangle \\
& \leq f(u)+\frac{1}{2 \eta}\left\|x^{(r+1)}-y^{(r)}\right\|_{2}^{2}+\frac{1}{\eta}\left\langle y^{(r)}-x^{(r+1)}, x^{(r+1)}-u\right\rangle .
\end{aligned}
$$


Combining these inequalities for $u:=\hat{x}$ and $u:=x^{(r)}$ with $\theta_{r} \in[0,1]$ gives

$$
\begin{aligned}
& \theta_{r}\left(f\left(x^{(r+1)}\right)-f(\hat{x})\right)+\left(1-\theta_{r}\right)\left(f\left(x^{(r+1)}\right)-f\left(x^{(r)}\right)\right) \\
& =f\left(x^{(r+1)}\right)-f(\hat{x})+\left(1-\theta_{r}\right)\left(f(\hat{x})-f\left(x^{(r)}\right)\right) \\
& \leq \frac{1}{2 \eta}\left\|x^{(r+1)}-y^{(r)}\right\|_{2}^{2}+\frac{1}{\eta}\left\langle y^{(r)}-x^{(r+1)}, x^{(r+1)}-\theta_{r} \hat{x}-\left(1-\theta_{r}\right) x^{(r)}\right\rangle \\
& =\frac{1}{2 \eta}\left(\left\|y^{(r)}-\theta_{r} \hat{x}-\left(1-\theta_{r}\right) x^{(r)}\right\|_{2}^{2}-\left\|x^{(r+1)}-\theta_{r} \hat{x}-\left(1-\theta_{r}\right) x^{(r)}\right\|_{2}^{2}\right) \\
& =\frac{\theta_{r}^{2}}{2 \eta}\left(\left\|z^{(r)}-\hat{x}\right\|_{2}^{2}-\left\|z^{(r+1)}-\hat{x}\right\|_{2}^{2}\right) .
\end{aligned}
$$

Thus, we obtain for a single step

$$
\frac{\eta}{\theta_{r}^{2}}\left(f\left(x^{(r+1)}\right)-f(\hat{x})\right)+\frac{1}{2}\left\|z^{(r+1)}-\hat{x}\right\|_{2}^{2} \leq \frac{\eta\left(1-\theta_{r}\right)}{\theta_{r}^{2}}\left(f\left(x^{(r)}-f(\hat{x})\right)+\frac{1}{2}\left\|z^{(r)}-\hat{x}\right\|_{2}^{2} .\right.
$$

Using the relation recursively on the right-hand side and regarding that $\frac{\left(1-\theta_{r}\right)}{\theta_{r}^{2}} \leq \frac{1}{\theta_{r-1}^{2}}$ we obtain

$$
\frac{\eta}{\theta_{r}^{2}}\left(f\left(x^{(r+1)}\right)-f(\hat{x})\right) \leq \frac{\eta\left(1-\theta_{0}\right)}{\theta_{0}^{2}}\left(f\left(x^{(0)}\right)-f(\hat{x})\right)+\frac{1}{2}\left\|z^{(0)}-\hat{x}\right\|_{2}^{2}=\frac{1}{2}\left\|x^{(0)}-\hat{x}\right\|_{2}^{2} .
$$

This yields the assertion

$$
f\left(x^{(r+1)}\right)-f(\hat{x}) \leq \frac{2}{\eta(r+2)^{2}}\left\|x^{(0)}-\hat{x}\right\|_{2}^{2}
$$

There exist many variants and generalizations of the above algorithm as

- Nesterov's algorithms [119, 121], see also [57, 164]; this includes approximation algorithms for nonsmooth $g$ [14, 122] as NESTA,

- fast iterative shrinkage algorithms (FISTA) by Beck and Teboulle [13,

- variable metric strategies [24, 33, 54, 131, where based on (5) step (19) is replaced by

$$
x^{(r+1)}=\operatorname{prox}_{Q_{r}, \eta_{r} h}\left(y^{(r)}-\eta_{r} Q_{r}^{-1} \nabla g\left(y^{(r)}\right)\right)
$$

with symmetric, positive definite matrices $Q_{r}$.

Line search strategies can be incorporated [83, 87, 120. Finally we mention Barzilei-Borwein step size rules [1] based on a Quasi-Newton approach and relatives, see [74] for an overview and the cyclic proximal gradient algorithm related to the cyclic Richardson algorithm [158]. 


\section{Primal-Dual Methods}

\subsection{Basic Relations}

The following minimization algorithms closely rely on the primal-dual formulation of problems. We consider functions $f=g+h(A \cdot)$, where $g \in \Gamma_{0}\left(\mathbb{R}^{d}\right), h \in \Gamma_{0}\left(\mathbb{R}^{m}\right)$, and $A \in \mathbb{R}^{m, d}$, and ask for the solution of the primal problem

$$
(P) \underset{x \in \mathbb{R}^{d}}{\operatorname{argmin}}\{g(x)+h(A x)\},
$$

that can be rewritten as

$$
\text { (P) } \underset{x \in \mathbb{R}^{d}, y \in \mathbb{R}^{m}}{\operatorname{argmin}}\{g(x)+h(y) \quad \text { s.t. } \quad A x=y\} .
$$

The Lagrangian of (24) is given by

$$
L(x, y, p):=g(x)+h(y)+\langle p, A x-y\rangle
$$

and the augmented Lagrangian by

$$
\begin{aligned}
L_{\gamma}(x, y, p) & :=g(x)+h(y)+\langle p, A x-y\rangle+\frac{\gamma}{2}\|A x-y\|_{2}^{2}, \quad \gamma>0, \\
& =g(x)+h(y)+\frac{\gamma}{2}\left\|A x-y+\frac{p}{\gamma}\right\|_{2}^{2}-\frac{1}{2 \gamma}\|p\|_{2}^{2} .
\end{aligned}
$$

Based on the Lagrangian 25), the primal and dual problem can be written as

$$
\begin{aligned}
& \text { (P) } \underset{x \in \mathbb{R}^{d}, y \in \mathbb{R}^{m}}{\operatorname{argmin}} \sup _{p \in \mathbb{R}^{m}}\{g(x)+h(y)+\langle p, A x-y\rangle\}, \\
& (D) \quad \underset{p \in \mathbb{R}^{m}}{\operatorname{argmax}} \inf _{x \in R^{d}, y \in \mathbb{R}^{m}}\{g(x)+h(y)+\langle p, A x-y\rangle\} .
\end{aligned}
$$

Since

$$
\min _{y \in \mathbb{R}^{m}}\{h(y)-\langle p, y\rangle\}=-\max _{y \in \mathbb{R}^{m}}\{\langle p, y\rangle-h(y)\}=-h^{*}(p)
$$

and in 23 further

$$
h(A x)=\max _{p \in \mathbb{R}^{m}}\left\{\langle p, A x\rangle-h^{*}(p)\right\}
$$

the primal and dual problem can be rewritten as

$$
\begin{aligned}
& (P) \quad \underset{x \in \mathbb{R}^{d}}{\operatorname{argmin}} \sup _{p \in \mathbb{R}^{m}}\left\{g(x)-h^{*}(p)+\langle p, A x\rangle\right\}, \\
& (D) \quad \underset{p \in \mathbb{R}^{m}}{\operatorname{argmax}} \inf _{x \in \mathbb{R}^{d}}\left\{g(x)-h^{*}(p)+\langle p, A x\rangle\right\} .
\end{aligned}
$$

If the infimum exists, the dual problem can be seen as Fenchel dual problem

$$
\text { (D) } \underset{p \in \mathbb{R}^{m}}{\operatorname{argmin}}\left\{g^{*}\left(-A^{\mathrm{T}} p\right)+h^{*}(p)\right\} .
$$

Recall that $((\hat{x}, \hat{y}), \hat{p}) \in \mathbb{R}^{d m, m}$ is a saddle point of the Lagrangian $L$ in $(25)$ if

$$
L((x, y), \hat{p}) \leq L((\hat{x}, \hat{y}), \hat{p}) \leq L((\hat{x}, \hat{y}), p) \quad \forall(x, y) \in \mathbb{R}^{d m}, p \in \mathbb{R}^{m} .
$$

If $((\hat{x}, \hat{y}), \hat{p}) \in \mathbb{R}^{d m, m}$ is a saddle point of $L$, then $(\hat{x}, \hat{y})$ is a solution of the primal problem (27) and $\hat{p}$ is a solution of the dual problem (28). The converse is also true. However the existence of a solution of the primal problem $(\hat{x}, \hat{y}) \in \mathbb{R}^{d m}$ does only imply under additional qualification constraint that there exists $\hat{p}$ such that $((\hat{x}, \hat{y}), \hat{p}) \in \mathbb{R}^{d m, m}$ is a saddle point of $L$. 


\subsection{Alternating Direction Method of Multipliers}

Based on the Lagrangian formulation (27) and (28), a first idea to solve the optimization problem would be to alternate the minimization of the Lagrangian with respect to $(x, y)$ and to apply a gradient ascent approach with respect respect to $p$. This is known as general Uzawa method [5]. More precisely, noting that for differentiable $\nu(p):=\inf _{x, y} L(x, y, p)=L(\tilde{x}, \tilde{y}, p)$ we have $\nabla \nu(p)=A \tilde{x}-\tilde{y}$, the algorithm reads

$$
\begin{aligned}
\left(x^{(r+1)}, y^{(r+1)}\right) & \in \underset{x \in \mathbb{R}^{d}, y \in \mathbb{R}^{m}}{\operatorname{argmin}} L\left(x, y, p^{(r)}\right), \\
p^{(r+1)} & =p^{(r)}+\gamma\left(A x^{(r+1)}-y^{(r+1)}\right), \quad \gamma>0 .
\end{aligned}
$$

Linear convergence can be proved under certain conditions (strict convexity of $f$ ) [81]. The assumptions on $f$ to ensure convergence of the algorithm can be relaxed by replacing the Lagrangian by the augmented Lagrangian $L_{\gamma}(26)$ with fixed parameter $\gamma$ :

$$
\begin{aligned}
\left(x^{(r+1)}, y^{(r+1)}\right) & \in \underset{x \in \mathbb{R}^{d}, y \in \mathbb{R}^{m}}{\operatorname{argmin}} L_{\gamma}\left(x, y, p^{(r)}\right), \\
p^{(r+1)} & =p^{(r)}+\gamma\left(A x^{(r+1)}-y^{(r+1)}\right), \quad \gamma>0 .
\end{aligned}
$$

This augmented Lagrangian method is known as method of multipliers [95, 134, 140]. It can be shown [35, Theorem 3.4.7], [17] that the sequence $\left(p^{(r)}\right)_{r}$ generated by the algorithm coincides with the proximal point algorithm applied to $-\nu(p)$, i.e.,

$$
p^{(r+1)}=\operatorname{prox}_{-\gamma \nu}\left(p^{(r)}\right) .
$$

The improved convergence properties came at a cost. While the minimization with respect to $x$ and $y$ can be separately computed in (30) using $\left\langle p^{(r)},(A \mid-I)\left(\begin{array}{l}x \\ y\end{array}\right)\right\rangle=\left\langle\left(\begin{array}{l}A^{\mathrm{T}} \\ -I\end{array}\right) p^{(r)},\left(\begin{array}{l}x \\ y\end{array}\right)\right\rangle$, this is no longer possible for the augmented Lagrangian. A remedy is to alternate the minimization with respect to $x$ and $y$ which leads to

$$
\begin{aligned}
& x^{(r+1)} \in \underset{x \in \mathbb{R}^{d}}{\operatorname{argmin}} L_{\gamma}\left(x, y^{(r)}, p^{(r)}\right), \\
& y^{(r+1)}=\underset{y \in \mathbb{R}^{m}}{\operatorname{argmin}} L_{\gamma}\left(x^{(r+1)}, y, p^{(r)}\right), \\
& p^{(r+1)}=p^{(r)}+\gamma\left(A x^{(r+1)}-y^{(r+1)}\right) .
\end{aligned}
$$

This is the alternating direction method of multipliers (ADMM) which dates back to [77, 78, 82 .

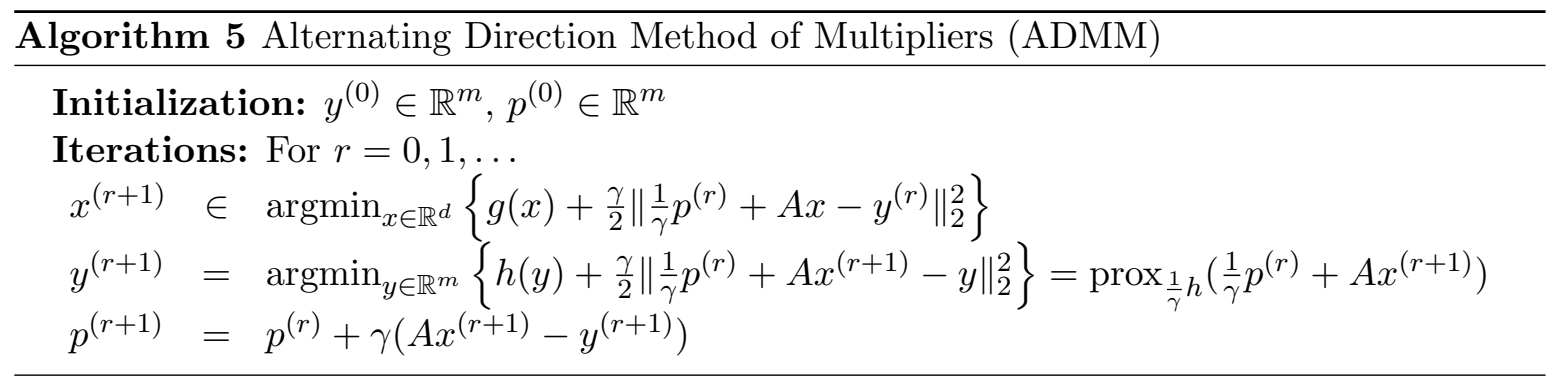


Setting $b^{(r)}:=p^{(r)} / \gamma$ we obtain the following (scaled) ADMM:

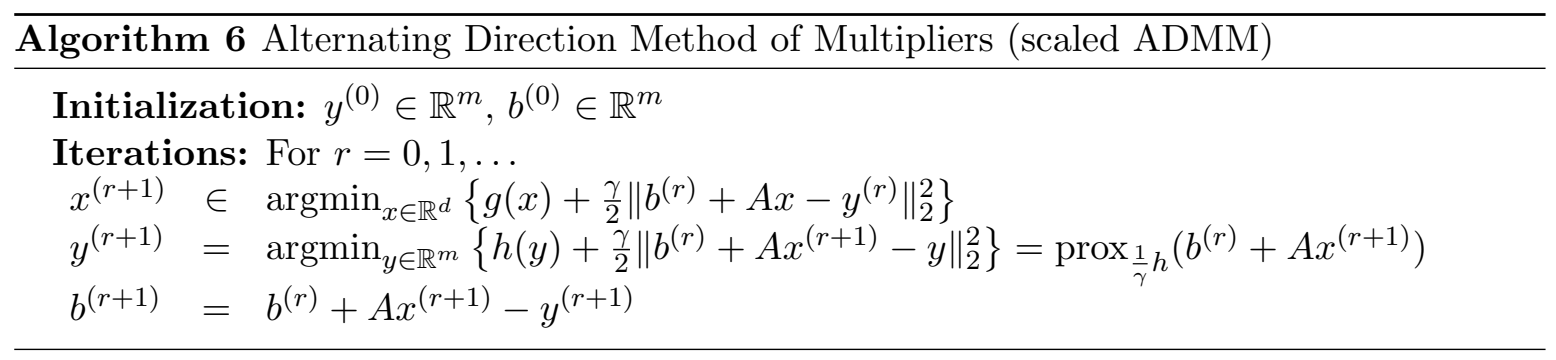

A good overview on the ADMM algorithm and its applications is given in [27, where in particular the important issue of choosing the parameter $\gamma>0$ is addressed. The ADMM can be considered for more general problems

$$
\underset{x \in \mathbb{R}^{d}, y \in \mathbb{R}^{m}}{\operatorname{argmin}}\{g(x)+h(y) \quad \text { s.t. } \quad A x+B y=c\} .
$$

Convergence of the ADMM under various assumptions was proved, e.g., in [78, 90, 109, 163]. We will see that for our problem (24) the convergence follows by the relation of the ADMM to the so-called Douglas-Rachford splitting algorithm which convergence can be shown using averaged operators. Few bounds on the global convergence rate of the algorithm can be found in [63] (linear convergence for linear programs depending on a variety of quantities), [96] (linear convergence for sufficiently small step size) and on the local behaviour of a specific variation of the ADMM during the course of iteration for quadratic programs in [21].

Theorem 6.1 (Convergence of ADMM). Let $g \in \Gamma_{0}\left(\mathbb{R}^{d}\right), h \in \Gamma_{0}\left(\mathbb{R}^{m}\right)$ and $A \in \mathbb{R}^{m, d}$. Assume that the Lagrangian 25) has a saddle point. Then, for $r \rightarrow \infty$, the sequence $\gamma\left(b^{(r)}\right)_{r}$ converges to a solution of the dual problem. If in addition the first step (32) in the ADMM algorithm has a unique solution, then $\left(x^{(r)}\right)_{r}$ converges to a solution of the primal problem.

There exist different modifications of the ADMM algorithm presented above:

- inexact computation of the first step (32) 45, 64, such that it might be handled by an iterative method,

- variable parameter and metric strategies [27, 89, 90, 92, 105] where the fixed parameter $\gamma$ can vary in each step, or the quadratic term $(\gamma / 2)\|A x-y\|_{2}^{2}$ within the augmented Lagrangian (26) is replaced by the more general proximal operator based on (5) such that the ADMM updates (32) and (33) receive the form

$$
\begin{aligned}
& x^{(r+1)} \in \underset{x \in \mathbb{R}^{d}}{\operatorname{argmin}}\left\{g(x)+\frac{1}{2}\left\|b^{(r)}+A x-y^{(r)}\right\|_{Q_{r}}^{2}\right\}, \\
& y^{(r+1)}=\underset{y \in \mathbb{R}^{m}}{\operatorname{argmin}}\left\{h(y)+\frac{1}{2}\left\|b^{(r)}+A x^{(r+1)}-y\right\|_{Q_{r}}^{2}\right\},
\end{aligned}
$$

respectively, with symmetric, positive definite matrices $Q_{r}$. The variable parameter strategies might mitigate the performance dependency on the initial chosen fixed parameter [27, 92, 105, 174] and include monotone conditions [90, 105] or more flexible non-monotone rules [27, 89, 92]. 
ADMM from the Perspective of Variational Inequalities The ADMM algorithm presented above from the perspective of Lagrangian functions has been also studied extensively in the area of variational inequalities (VIs), see, e.g., [77, 89, 163]. A VI problem consists of finding for a mapping $F: \mathbb{R}^{l} \rightarrow \mathbb{R}^{l}$ a vector $\hat{z} \in \mathbb{R}^{l}$ such that

$$
\langle z-\hat{z}, F(\hat{z})\rangle \geq 0, \quad \forall z \in \mathbb{R}^{l} .
$$

In the following, we consider the minimization problem (24), i.e.,

$$
\underset{x \in \mathbb{R}^{d}, y \in \mathbb{R}^{m}}{\operatorname{argmin}}\{g(x)+h(y) \text { s.t. } A x=y\},
$$

for $g \in \Gamma_{0}\left(\mathbb{R}^{d}\right), h \in \Gamma_{0}\left(\mathbb{R}^{m}\right)$. The discussion can be extended to the more general problem (34) [89, 163]. Considering the Lagrangian (25) and its optimality conditions, solving (24) is equivalent to find a triple $\hat{z}=((\hat{x}, \hat{y}), \hat{p}) \in \mathbb{R}^{d m, m}$ such that 35 holds with

$$
z=\left(\begin{array}{c}
x \\
y \\
p
\end{array}\right), \quad F(z)=\left(\begin{array}{c}
\partial g(x)+A^{\mathrm{T}} p \\
\partial h(y)-p \\
A x-y
\end{array}\right)
$$

where $\partial g$ and $\partial h$ have to be understood as any element of the corresponding subdifferential for simplicity. Note that $\partial g$ and $\partial h$ are maximal monotone operators [12]. A VI problem of this form can be solved by ADMM as proposed by Gabay [77] and Gabay and Mercier [78]: for a given triple $\left(x^{(r)}, y^{(r)}, p^{(r)}\right)$ generate new iterates $\left(x^{(r+1)}, y^{(r+1)}, p^{(r+1)}\right)$ by

i) find $x^{(r+1)}$ such that

$$
\left\langle x-x^{(r+1)}, \partial g\left(x^{(r+1)}\right)+A^{\mathrm{T}}\left(p^{(r)}+\gamma\left(A x^{(r+1)}-y^{(r)}\right)\right)\right\rangle \geq 0, \quad \forall x \in \mathbb{R}^{d},
$$

ii) find $y^{(r+1)}$ such that

$$
\left\langle y-y^{(r+1)}, \partial h\left(y^{(r+1)}\right)-\left(p^{(r)}+\gamma\left(A x^{(r+1)}-y^{(r+1)}\right)\right)\right\rangle \geq 0, \quad \forall y \in \mathbb{R}^{m},
$$

iii) update $p^{(r+1)}$ via

$$
p^{(r+1)}=p^{(r)}+\gamma\left(A x^{(r+1)}-y^{(r+1)}\right),
$$

where $\gamma>0$ is a fixed penalty parameter. To corroborate the equivalence of the iteration scheme above to ADMM in Algorithm 5, note that (35) reduces to $\langle\hat{z}, F(\hat{z})\rangle \geq 0$ for $z=2 \hat{z}$. On the other hand, (35) is equal to $\langle\hat{z}, F(\hat{z})\rangle \leq 0$ when $z=-\hat{z}$. The both cases transform (35) to find a solution $\hat{z}$ of a system of equations $F(\hat{z})=0$. Thus, the VI sub-problems (36) and (37) can be reduced to find a pair $\left(x^{(r+1)}, y^{(r+1)}\right)$ with

$$
\begin{aligned}
\partial g\left(x^{(r+1)}\right)+A^{\mathrm{T}}\left(p^{(r)}+\gamma\left(A x^{(r+1)}-y^{(r)}\right)\right) & =0, \\
\partial h\left(y^{(r+1)}\right)-\left(p^{(r)}+\gamma\left(A x^{(r+1)}-y^{(r+1)}\right)\right) & =0 .
\end{aligned}
$$

The both equations correspond to optimality conditions of the minimization sub-problems (32) and (33) of the ADMM algorithm, respectively. The theoretical properties of ADMM from the perspective of VI problems were studied extensively and a good reference overview can be found in 89 . 
Relation to Douglas-Rachford Splitting Finally we want to point out the relation of the ADMM to the Douglas-Rachford splitting algorithm applied to the dual problem, see [64, 65, 77, 157]. We consider again the problem (17), i.e.,

$$
\underset{x \in \mathbb{R}^{d}}{\operatorname{argmin}}\{g(x)+h(x)\},
$$

where we assume this time only $g, h \in \Gamma_{0}\left(\mathbb{R}^{d}\right)$ and that $f$ or $g$ is continuous at a point in $\operatorname{dom} g \cap \operatorname{dom} h$. Fermat's rule and subdifferential calculus implies that $\hat{x}$ is a minimizer if and only if

$$
0 \in \partial g(\hat{x})+\partial h(\hat{x}) \quad \Leftrightarrow \quad \exists \hat{\xi} \in \eta \partial g(\hat{x}) \text { such that } \hat{x}=\operatorname{prox}_{\eta h}(\hat{x}-\hat{\xi}) .
$$

The basic idea for finding such minimizer is to set up a 'nice' operator $T: \mathbb{R}^{d} \rightarrow \mathbb{R}^{d}$ by

$$
T:=\operatorname{prox}_{\eta h}\left(2 \operatorname{prox}_{\eta g}-I\right)-\operatorname{prox}_{\eta g}+I,
$$

which fixed points $\hat{t}$ are related to the minimizers as follows: setting $\hat{x}:=\operatorname{prox}_{\eta g}(\hat{t})$, i.e., $\hat{t} \in \hat{x}+\eta \partial g(\hat{x})$ and $\hat{\xi}:=\hat{t}-\hat{x} \in \eta \partial g(\hat{x})$ we see that

$$
\begin{aligned}
\hat{t} & =T(\hat{t})=\operatorname{prox}_{\eta h}(2 \hat{x}-\hat{t})-\hat{x}+\hat{t}, \\
\hat{\xi}+\hat{x} & =\operatorname{prox}_{\eta h}(\hat{x}-\hat{\xi})+\hat{\xi}, \\
\hat{x} & =\operatorname{prox}_{\eta h}(\hat{x}-\hat{\xi}),
\end{aligned}
$$

which coincides with (38). By the proof of the next theorem, the operator $T$ is firmly nonexpansive such that by Theorem 4.7 a fixed point of $T$ can be found by Picard iterations. This gives rise to the following Douglas-Rachford splitting algorithm (DRS).

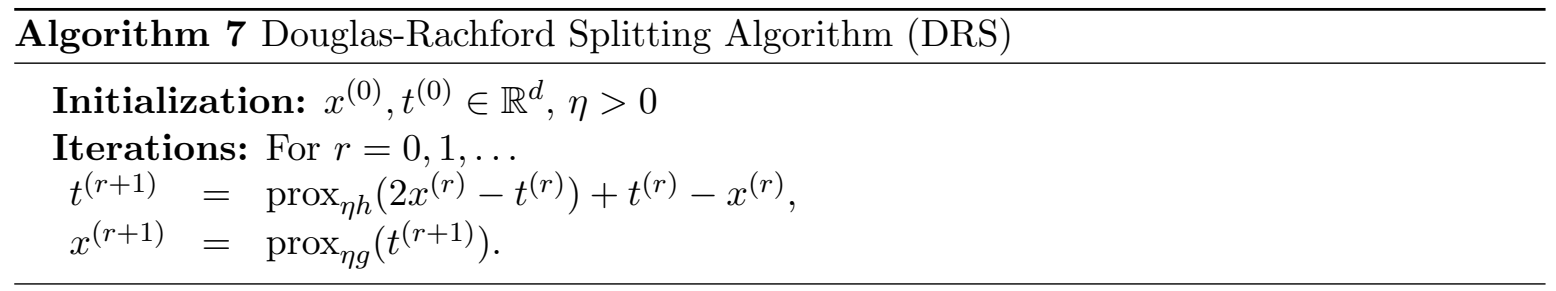

The following theorem verifies the convergence of the DRS algorithm. For a recent convergence result see also 91 .

Theorem 6.2 (Convergence of Douglas-Rachford Splitting Algorithm). Let $g, h \in \Gamma_{0}\left(\mathbb{R}^{d}\right)$ where one of the functions is continuous at a point in $\operatorname{dom} g \cap \operatorname{dom} h$. Assume that a solution of $\operatorname{argmin}_{x \in \mathbb{R}^{d}}\{g(x)+h(x)\}$ exists. Then, for any initial $t^{(0)}, x^{(0)} \in \mathbb{R}^{d}$ and any $\eta>0$, the DRS sequence $\left(t^{(r)}\right)_{r}$ converges to a fixed point $\hat{t}$ of $T$ in $(39)$ and $\left(x^{(r)}\right)_{r}$ to a solution of the minimization problem.

Proof. It remains to show that $T$ is firmly nonexpansive. We have for $R_{1}:=2 \operatorname{prox}_{\eta g}-I$ and $R_{2}:=2 \operatorname{prox}_{\eta h}-I$ that

$$
\begin{aligned}
2 T & =2 \operatorname{prox}_{\eta h}\left(2 \operatorname{prox}_{\eta g}-I\right)-\left(2 \operatorname{prox}_{\eta g}-I\right)+I=R_{2} \circ R_{1}+I, \\
T & =\frac{1}{2} I+\frac{1}{2} R_{2} \circ R_{1} .
\end{aligned}
$$

The operators $R_{i}, i=1,2$ are nonexpansive since $\operatorname{prox}_{\eta g}$ and prox $_{\eta h}$ are firmly nonexpansive. Hence $R_{2} \circ R_{1}$ is nonexpansive and we are done. 
The relation of the ADMM algorithm and DRS algorithm applied to the Fenchel dual problem $(29)$, i.e.,

$$
\begin{aligned}
& t^{(r+1)}=\operatorname{prox}_{\eta g^{*} \circ\left(-A^{\mathrm{T}}\right)}\left(2 p^{(r)}-t^{(r)}\right)+t^{(r)}-p^{(r)}, \\
& p^{(r+1)}=\operatorname{prox}_{\eta h^{*}}\left(t^{(r+1)}\right),
\end{aligned}
$$

is given by the following theorem, see [64, 77].

Theorem 6.3 (Relation between ADMM and DRS). The ADMM sequences $\left(b^{(r)}\right)_{r}$ and $\left(y^{(r)}\right)_{r}$ are related with the sequences 40 generated by the DRS algorithm applied to the dual problem by $\eta=\gamma$ and

$$
\begin{aligned}
& t^{(r)}=\eta\left(b^{(r)}+y^{(r)}\right), \\
& p^{(r)}=\eta b^{(r)} .
\end{aligned}
$$

Proof. First, we show that

$$
\hat{p}=\underset{p \in \mathbb{R}^{m}}{\operatorname{argmin}}\left\{\frac{\eta}{2}\|A p-q\|_{2}^{2}+g(p)\right\} \quad \Rightarrow \quad \eta(A \hat{p}-q)=\operatorname{prox}_{\eta g^{*} \circ\left(-A^{\mathrm{T}}\right)}(-\eta q)
$$

holds true. The left-hand side of 42 is equivalent to

$$
0 \in \eta A^{\mathrm{T}}(A \hat{p}-q)+\partial g(\hat{p}) \quad \Leftrightarrow \quad \hat{p} \in \partial g^{*}\left(-\eta A^{\mathrm{T}}(A \hat{p}-q)\right) .
$$

Applying $-\eta A$ on both sides and using the chain rule implies

$$
-\eta A \hat{p} \in-\eta A \partial g^{*}\left(-\eta A^{\mathrm{T}}(A \hat{p}-q)\right)=\eta \partial\left(g^{*} \circ\left(-A^{\mathrm{T}}\right)\right)(\eta(A \hat{p}-q)) .
$$

Adding $-\eta q$ we get

$$
-\eta q \in\left(I+\eta \partial\left(g^{*} \circ\left(-A^{\mathrm{T}}\right)\right)\right)(\eta(A \hat{p}-q)),
$$

which is equivalent to the right-hand side of (42) by the definition of the resolvent (see Remark 3.4.

Secondly, applying (42) to the first ADMM step with $\gamma=\eta$ and $q:=y^{(r)}-b^{(r)}$ yields

$$
\eta\left(b^{(r)}+A x^{(r+1)}-y^{(r)}\right)=\operatorname{prox}_{\eta g^{*} \circ\left(-A^{\mathrm{T}}\right)}\left(\eta\left(b^{(r)}-y^{(r)}\right)\right) .
$$

Assume that the ADMM and DRS iterates have the identification 41) up to some $r \in \mathbb{N}$. Using this induction hypothesis it follows that

$$
\eta\left(b^{(r)}+A x^{(r+1)}\right)=\operatorname{prox}_{\eta g^{*} \circ\left(-A^{\mathrm{T}}\right)}(\underbrace{\eta\left(b^{(r)}-y^{(r)}\right)}_{2 p^{(r)}-t^{(r)}})+\underbrace{\eta y^{(r)}}_{t^{(r)}-p^{(r)}} \stackrel{40)}{=} t^{(r+1)} .
$$

By definition of $b^{(r+1)}$ we see that $\eta\left(b^{(r+1)}+y^{(r+1)}\right)=t^{(r+1)}$. Next we apply (42) in the second ADMM step where we replace $g$ by $h$ and $A$ by $-I$ and use $q:=-b^{(r)}-A x^{(r+1)}$. Together with $(43)$ this gives

$$
\eta\left(-y^{(r+1)}+b^{(r)}+A x^{(r+1)}\right)=\operatorname{prox}_{\eta h^{*}}(\underbrace{\eta\left(b^{(r)}+A x^{(r+1)}\right)}_{t^{(r+1)}}) \stackrel{\text { 400 }}{=} p^{(r+1)} .
$$

Using again the definition of $b^{(r+1)}$ we obtain $\eta b^{(r+1)}=p^{(r+1)}$ which completes the proof. 


\subsection{Primal Dual Hybrid Gradient Algorithms}

The first ADMM step (32) requires in general the solution of a linear system of equations. This can be avoided by modifying this step using the Taylor expansion at $x^{(r)}$ :

$\frac{\gamma}{2}\left\|\frac{1}{\gamma} p^{(r)}+A x-y^{(r)}\right\|_{2}^{2} \approx$ const $+\gamma\left\langle A^{\mathrm{T}}\left(A x^{(r)}-y^{(r)}+\frac{1}{\gamma} p^{(r)}\right), x\right\rangle+\frac{\gamma}{2}\left(x-x^{(r)}\right)^{\mathrm{T}} A^{\mathrm{T}} A\left(x-x^{(r)}\right)$, approximating $A^{\mathrm{T}} A \approx \frac{1}{\gamma \tau} I$, setting $\gamma:=\sigma$ and using $p^{(r)} / \sigma$ instead of $A x^{(r)}-y^{(r)}+p^{(r)} / \sigma$ we obtain (note that $\left.p^{(r+1)} / \sigma=p^{(r)} / \sigma+A x^{(r+1)}-y^{(r+1)}\right)$ :

$$
\begin{aligned}
& x^{(r+1)}=\operatorname{argmin}_{x \in \mathbb{R}^{d}}\left\{g(x)+\frac{1}{2 \tau}\left\|x-\left(x^{(r)}-\tau A^{\mathrm{T}} p^{(r)}\right)\right\|_{2}^{2}\right\}=\operatorname{prox}_{\tau g}\left(x^{(r)}-\tau A^{\mathrm{T}} p^{(r)}\right), \\
& y^{(r+1)}=\operatorname{argmin}_{y \in \mathbb{R}^{n}}\left\{h(y)+\frac{\sigma}{2}\left\|\frac{1}{\sigma} p^{(r)}+A x^{(r+1)}-y\right\|_{2}^{2}\right\}=\operatorname{prox}_{\frac{1}{\sigma} h}\left(\frac{1}{\sigma} p^{(r)}+A x^{(r+1)}\right), \\
& p^{(r+1)}=p^{(r)}+\sigma\left(A x^{(r+1)}-y^{(r+1)}\right) .
\end{aligned}
$$

The above algorithm can be deduced in another way by the Arrow-Hurwicz method: we alternate the minimization in the primal and dual problems (27) and (28) and add quadratic terms. The resulting sequences

$$
\begin{aligned}
x^{(r+1)} & =\underset{x \in \mathbb{R}^{d}}{\operatorname{argmin}}\left\{g(x)+\left\langle p^{(r)}, A x\right\rangle+\frac{1}{2 \tau}\left\|x-x^{(r)}\right\|_{2}^{2}\right\}, \\
& =\operatorname{prox}_{\tau g}\left(x^{(r)}-\tau A^{\mathrm{T}} p^{(r)}\right) \\
p^{(r+1)} & =\underset{p \in \mathbb{R}^{m}}{\operatorname{argmin}}\left\{h^{*}(p)-\left\langle p, A x^{(r+1)}\right\rangle+\frac{1}{2 \sigma}\left\|p-p^{(r)}\right\|_{2}^{2}\right\}, \\
& =\operatorname{prox}_{\sigma h^{*}}\left(p^{(r)}+\sigma A x^{(r+1)}\right)
\end{aligned}
$$

coincide with those in (45) which can be seen as follows: For $x^{(r)}$ the relation is straightforward. From the last equation we obtain

$$
\begin{aligned}
p^{(r)}+\sigma A x^{(r+1)} & \in p^{(r+1)}+\sigma \partial h^{*}\left(p^{(r+1)}\right), \\
\frac{1}{\sigma}\left(p^{(r)}-p^{(r+1)}\right)+A x^{(r+1)} & \in \partial h^{*}\left(p^{(r+1)}\right),
\end{aligned}
$$

and using that $p \in \partial h(x) \Leftrightarrow x \in \partial h^{*}(p)$ further

$$
p^{(r+1)} \in \partial h(\underbrace{\frac{1}{\sigma}\left(p^{(r)}-p^{(r+1)}\right)+A x^{(r+1)}}_{y^{(r+1)}}) .
$$

Setting

$$
y^{(r+1)}:=\frac{1}{\sigma}\left(p^{(r)}-p^{(r+1)}\right)+A x^{(r+1)},
$$

we get

$$
p^{(r+1)}=p^{(r)}+\sigma\left(A x^{(r+1)}-y^{(r+1)}\right)
$$

and $p^{(r+1)} \in \partial h\left(y^{(r+1)}\right)$ which can be rewritten as

$$
\begin{aligned}
y^{(r+1)}+\frac{1}{\sigma} p^{(r+1)} & \in y^{(r+1)}+\frac{1}{\sigma} \partial h\left(y^{(r+1)}\right), \\
\frac{1}{\sigma} p^{(r)}+A x^{(r+1)} & \in y^{(r+1)}+\frac{1}{\sigma} \partial h\left(y^{(r+1)}\right), \\
y^{(r+1)} & =\operatorname{prox}_{\frac{1}{\sigma} h}\left(\frac{1}{\sigma} p^{(r)}+A x^{(r+1)}\right) .
\end{aligned}
$$


There are several modifications of the basic linearized ADMM which improve its convergence properties as

- the predictor corrector proximal multiplier method [45],

- the primal dual hybrid gradient method (PDHG) [182] with convergence proof in [23],

- primal dual hybrid gradient method with extrapolation of the primal or dual variable [42, 133], a preconditioned version [41] and a generalization [55], Douglas-Rachford-type algorithms [25, 26] for solving inclusion equations, see also [51, 170], as well an extension allowing the operator $A$ to be non-linear [165].

A good overview on primal-dual methods is also given in [104]. Here is the algorithm proposed by Chambolle, Cremers and Pock [42, 133].

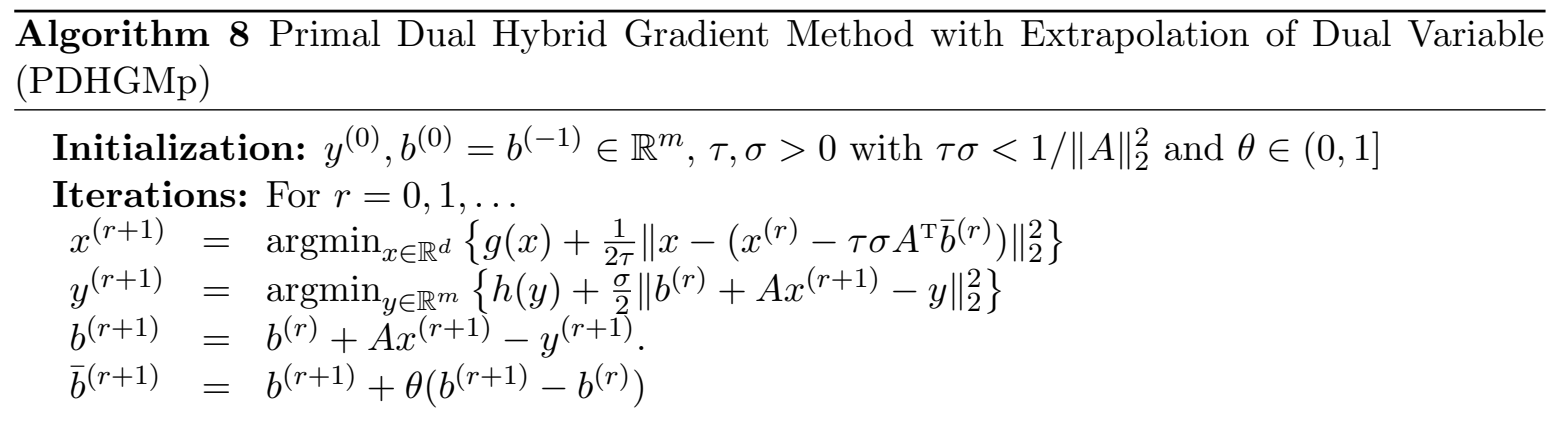

Note that the new first updating step can be also deduced by applying the so-called inexact Uzawa algorithm to the first ADMM step (see Section 6.4). Furthermore, it can be directly seen that for $A$ being the identity and $\theta=1$ and $\gamma=\sigma=\frac{1}{\tau}$, the PDHGMp algorithm corresponds to the ADMM as well as the Douglas-Rachford splitting algorithm as proposed in Section 6.2. The following theorem and convergence proof are based on [42].

Theorem 6.4. Let $g \in \Gamma_{0}\left(\mathbb{R}^{d}\right), h \in \Gamma_{0}\left(\mathbb{R}^{m}\right)$ and $\theta \in(0,1]$. Let $\tau, \sigma>0$ fulfill

$$
\tau \sigma<1 /\|A\|_{2}^{2} .
$$

Suppose that the Lagrangian $L(x, p):=g(x)-h^{*}(p)+\langle A x, p\rangle$ has a saddle point $\left(x^{*}, p^{*}\right)$. Then the sequence $\left\{\left(x^{(r)}, p^{(r)}\right)\right\}_{r}$ produced by PDGHMp converges to a saddle point of the Lagrangian.

Proof. We restrict the proof to the case $\theta=1$. For arbitrary $\bar{x} \in \mathbb{R}^{d}, \bar{p} \in \mathbb{R}^{m}$ consider according to 46) and (47) the iterations

$$
\begin{aligned}
& x^{(r+1)}=(I+\tau \partial g)^{-1}\left(x^{(r)}-\tau A^{\mathrm{T}} \bar{p}\right), \\
& p^{(r+1)}=\left(I+\sigma \partial h^{*}\right)^{-1}\left(p^{(r)}+\sigma A \bar{x}\right),
\end{aligned}
$$

i.e.,

$$
\frac{x^{(r)}-x^{(r+1)}}{\tau}-A^{\mathrm{T}} \bar{p} \in \partial g\left(x^{(r+1)}\right), \quad \frac{p^{(r)}-p^{(r+1)}}{\sigma}+A \bar{x} \in \partial h^{*}\left(p^{(r+1)}\right) .
$$


By definition of the subdifferential we obtain for all $x \in \mathbb{R}^{d}$ and all $p \in \mathbb{R}^{m}$ that

$$
\begin{aligned}
g(x) & \geq g\left(x^{(r+1)}\right)+\frac{1}{\tau}\left\langle x^{(r)}-x^{(r+1)}, x-x^{(r+1)}\right\rangle-\left\langle A^{\mathrm{T}} \bar{p}, x-x^{(r+1)}\right\rangle, \\
h^{*}(p) & \geq h^{*}\left(p^{(r+1)}\right)+\frac{1}{\sigma}\left\langle p^{(r)}-p^{(r+1)}, p-p^{(r+1)}\right\rangle+\left\langle p-p^{(r+1)}, A \bar{x}\right\rangle
\end{aligned}
$$

and by adding the equations

$$
\begin{aligned}
0 \geq & g\left(x^{(r+1)}\right)-h^{*}(p)-\left(g(x)-h^{*}\left(p^{(r+1)}\right)\right)-\left\langle A^{\mathrm{T}} \bar{p}, x-x^{(r+1)}\right\rangle+\left\langle p-p^{(r+1)}, A \bar{x}\right\rangle \\
& +\frac{1}{\tau}\left\langle x^{(r)}-x^{(r+1)}, x-x^{(r+1)}\right\rangle+\frac{1}{\sigma}\left\langle p^{(r)}-p^{(r+1)}, p-p^{(r+1)}\right\rangle .
\end{aligned}
$$

By

$$
\left\langle x^{(r)}-x^{(r+1)}, x-x^{(r+1)}\right\rangle=\frac{1}{2}\left(\left\|x^{(r)}-x^{(r+1)}\right\|_{2}^{2}+\left\|x-x^{(r+1)}\right\|_{2}^{2}-\left\|x-x^{(r)}\right\|_{2}^{2}\right)
$$

this can be rewritten as

$$
\begin{aligned}
& \frac{1}{2 \tau}\left\|x-x^{(r)}\right\|_{2}^{2}+\frac{1}{2 \sigma}\left\|p-p^{(r)}\right\|_{2}^{2} \\
\geq & \frac{1}{2 \tau}\left\|x^{(r)}-x^{(r+1)}\right\|_{2}^{2}+\frac{1}{2 \tau}\left\|x-x^{(r+1)}\right\|_{2}^{2}+\frac{1}{2 \sigma}\left\|p^{(r)}-p^{(r+1)}\right\|_{2}^{2}+\frac{1}{2 \sigma}\left\|p-p^{(r+1)}\right\|_{2}^{2} \\
& +\left(g\left(x^{(r+1)}\right)-h^{*}(p)+\left\langle p, A x^{(r+1)}\right\rangle\right)-\left(g(x)-h^{*}\left(p^{(r+1)}\right)+\left\langle p^{(r+1)}, A x\right\rangle\right) \\
& -\left\langle p, A x^{(r+1)}\right\rangle+\left\langle p^{(r+1)}, A x\right\rangle-\left\langle\bar{p}, A\left(x-x^{(r+1)}\right)\right\rangle+\left\langle p-p^{(r+1)}, A \bar{x}\right\rangle \\
= & \frac{1}{2 \tau}\left\|x^{(r)}-x^{(r+1)}\right\|_{2}^{2}+\frac{1}{2 \tau}\left\|x-x^{(r+1)}\right\|_{2}^{2}+\frac{1}{2 \sigma}\left\|p^{(r)}-p^{(r+1)}\right\|_{2}^{2}+\frac{1}{2 \sigma}\left\|p-p^{(r+1)}\right\|_{2}^{2} \\
& +\left(g\left(x^{(r+1)}\right)-h^{*}(p)+\left\langle p, A x^{(r+1)}\right\rangle\right)-\left(g(x)-h^{*}\left(p^{(r+1)}\right)+\left\langle p^{(r+1)}, A x\right\rangle\right) \\
& +\left\langle p^{(r+1)}-p, A\left(x^{(r+1)}-\bar{x}\right)\right\rangle-\left\langle p^{(r+1)}-\bar{p}, A\left(x^{(r+1)}-x\right)\right\rangle .
\end{aligned}
$$

For any saddle point $\left(x^{*}, p^{*}\right)$ we have that $L\left(x^{*}, p\right) \leq L\left(x^{*}, p^{*}\right) \leq L\left(x, p^{*}\right)$ for all $x, p$ so that in particular $0 \leq L\left(x^{(r+1)}, p^{*}\right)-L\left(x^{*}, p^{(r+1)}\right)$. Thus, using $(x, p):=\left(x^{*}, p^{*}\right)$ in the above inequality, we get

$$
\begin{aligned}
& \frac{1}{2 \tau}\left\|x^{*}-x^{(r)}\right\|_{2}^{2}+\frac{1}{2 \sigma}\left\|p^{*}-p^{(r)}\right\|_{2}^{2} \\
\geq & \frac{1}{2 \tau}\left\|x^{(r)}-x^{(r+1)}\right\|_{2}^{2}+\frac{1}{2 \tau}\left\|x^{*}-x^{(r+1)}\right\|_{2}^{2}+\frac{1}{2 \sigma}\left\|p^{(r)}-p^{(r+1)}\right\|_{2}^{2}+\frac{1}{2 \sigma}\left\|p^{*}-p^{(r+1)}\right\|_{2}^{2} \\
& +\left\langle p^{(r+1)}-p^{*}, A\left(x^{(r+1)}-\bar{x}\right)\right\rangle-\left\langle p^{(r+1)}-\bar{p}, A\left(x^{(r+1)}-x^{*}\right)\right\rangle .
\end{aligned}
$$

In the algorithm we use $\bar{x}:=x^{(r+1)}$ and $\bar{p}:=2 p^{(r)}-p^{(r-1)}$. Note that $\bar{p}=p^{(r+1)}$ would be the better choice, but this is impossible if we want to keep on an explicit algorithm. For these values the above inequality further simplifies to

$$
\begin{aligned}
& \frac{1}{2 \tau}\left\|x^{*}-x^{(r)}\right\|_{2}^{2}+\frac{1}{2 \sigma}\left\|p^{*}-p^{(r)}\right\|_{2}^{2} \\
\geq & \frac{1}{2 \tau}\left\|x^{(r)}-x^{(r+1)}\right\|_{2}^{2}+\frac{1}{2 \tau}\left\|x^{*}-x^{(r+1)}\right\|_{2}^{2}+\frac{1}{2 \sigma}\left\|p^{(r)}-p^{(r+1)}\right\|_{2}^{2}+\frac{1}{2 \sigma}\left\|p^{*}-p^{(r+1)}\right\|_{2}^{2} \\
& +\left\langle p^{(r+1)}-2 p^{(r)}+p^{(r-1)}, A\left(x^{*}-x^{(r+1)}\right)\right\rangle .
\end{aligned}
$$


We estimate the last summand using Cauchy-Schwarz's inequality as follows:

$$
\begin{aligned}
& \left\langle p^{(r+1)}-p^{(r)}-\left(p^{(r)}-p^{(r-1)}\right), A\left(x^{*}-x^{(r+1)}\right)\right\rangle \\
= & \left\langle p^{(r+1)}-p^{(r)}, A\left(x^{*}-x^{(r+1)}\right)\right\rangle-\left\langle p^{(r)}-p^{(r-1)}, A\left(x^{*}-x^{(r)}\right)\right\rangle \\
& -\left\langle p^{(r)}-p^{(r-1)}, A\left(x^{(r)}-x^{(r+1)}\right)\right\rangle \\
\geq & \left\langle p^{(r+1)}-p^{(r)}, A\left(x^{*}-x^{(r+1)}\right)\right\rangle-\left\langle p^{(r)}-p^{(r-1)}, A\left(x^{*}-x^{(r)}\right)\right\rangle \\
& -\|A\|_{2}\left\|x^{(r+1)}-x^{(r)}\right\|_{2}\left\|p^{(r)}-p^{(r-1)}\right\|_{2} .
\end{aligned}
$$

Since

$$
2 u v \leq \alpha u^{2}+\frac{1}{\alpha} v^{2}, \quad \alpha>0
$$

we obtain

$$
\begin{aligned}
\|A\|_{2}\left\|x^{(r+1)}-x^{(r)}\right\|_{2}\left\|p^{(r)}-p^{(r-1)}\right\|_{2} & \leq \frac{\|A\|_{2}}{2}\left(\alpha\left\|x^{(r+1)}-x^{(r)}\right\|_{2}^{2}+\frac{1}{\alpha}\left\|p^{(r)}-p^{(r-1)}\right\|_{2}^{2}\right) \\
& =\frac{\|A\|_{2} \alpha \tau}{2 \tau}\left\|x^{(r+1)}-x^{(r)}\right\|_{2}^{2}+\frac{\|A\|_{2} \sigma}{2 \alpha \sigma}\left\|p^{(r)}-p^{(r-1)}\right\|_{2}^{2} .
\end{aligned}
$$

With $\alpha:=\sqrt{\sigma / \tau}$ the relation

$$
\|A\|_{2} \alpha \tau=\frac{\|A\|_{2} \sigma}{\alpha}=\|A\|_{2} \sqrt{\sigma \tau}<1
$$

holds true. Thus, we get

$$
\begin{aligned}
& \frac{1}{2 \tau}\left\|x^{*}-x^{(r)}\right\|_{2}^{2}+\frac{1}{2 \sigma}\left\|p^{*}-p^{(r)}\right\|_{2}^{2} \\
\geq & \frac{1}{2 \tau}\left\|x^{*}-x^{(r+1)}\right\|_{2}^{2}+\frac{1}{2 \sigma}\left\|p^{*}-p^{(r+1)}\right\|_{2}^{2} \\
& +\frac{1}{2 \tau}\left(1-\|A\|_{2} \sqrt{\sigma \tau}\right)\left\|x^{(r+1)}-x^{(r)}\right\|_{2}^{2}+\frac{1}{2 \sigma}\left\|p^{(r+1)}-p^{(r)}\right\|_{2}^{2}-\frac{\|A\|_{2} \sqrt{\sigma \tau}}{2 \sigma}\left\|p^{(r)}-p^{(r-1)}\right\|_{2}^{2} \\
& +\left\langle p^{(r+1)}-p^{(r)}, A\left(x^{*}-x^{(r+1)}\right)\right\rangle-\left\langle p^{(r)}-p^{(r-1)}, A\left(x^{*}-x^{(r)}\right)\right\rangle .
\end{aligned}
$$

Summing up these inequalities from $r=0$ to $N-1$ and regarding that $p^{(0)}=p^{(-1)}$, we obtain

$$
\begin{aligned}
& \frac{1}{2 \tau}\left\|x^{*}-x^{(0)}\right\|_{2}^{2}+\frac{1}{2 \sigma}\left\|p^{*}-p^{(0)}\right\|_{2}^{2} \\
\geq & \frac{1}{2 \tau}\left\|x^{*}-x^{(N)}\right\|_{2}^{2}+\frac{1}{2 \sigma}\left\|p^{*}-p^{(N)}\right\|_{2}^{2} \\
& +\left(1-\|A\|_{2} \sqrt{\sigma \tau}\right)\left(\frac{1}{2 \tau} \sum_{r=1}^{N}\left\|x^{(r)}-x^{(r-1)}\right\|_{2}^{2}+\frac{1}{2 \sigma} \sum_{r=1}^{N-1}\left\|p^{(r)}-p^{(r-1)}\right\|_{2}^{2}\right) \\
& +\frac{1}{2 \sigma}\left\|p^{(N)}-p^{(N-1)}\right\|_{2}^{2}+\left\langle p^{(N)}-p^{(N-1)}, A\left(x^{*}-x^{(N)}\right)\right\rangle
\end{aligned}
$$

By

$$
\left\langle p^{(N)}-p^{(N-1)}, A\left(x^{(N)}-x^{*}\right)\right\rangle \leq \frac{1}{2 \sigma}\left\|p^{(N)}-p^{(N-1)}\right\|_{2}^{2}+\frac{\|A\|_{2}^{2} \sigma \tau}{2 \tau}\left\|x^{(N)}-x^{*}\right\|_{2}^{2}
$$


this can be further estimated as

$$
\begin{aligned}
& \frac{1}{2 \tau}\left\|x^{*}-x^{(0)}\right\|_{2}^{2}+\frac{1}{2 \sigma}\left\|p^{*}-p^{(0)}\right\|_{2}^{2} \\
\geq & \frac{1}{2 \tau}\left(1-\|A\|_{2}^{2} \sigma \tau\right)\left\|x^{*}-x^{(N)}\right\|_{2}^{2}+\frac{1}{2 \sigma}\left\|p^{*}-p^{(N)}\right\|_{2}^{2} \\
& +\left(1-\|A\|_{2} \sqrt{\sigma \tau}\right)\left(\frac{1}{2 \tau} \sum_{k=1}^{N}\left\|x^{(r)}-x^{(r-1)}\right\|_{2}^{2}+\frac{1}{2 \sigma} \sum_{k=1}^{N-1}\left\|p^{(r)}-p^{(r-1)}\right\|_{2}^{2}\right) .
\end{aligned}
$$

By (52) we conclude that the sequence $\left\{\left(x^{(n)}, p^{(n)}\right)\right\}_{n}$ is bounded. Thus, there exists a convergent subsequence $\left\{\left(x^{\left(n_{j}\right)}, p^{\left(n_{j}\right)}\right)\right\}_{j}$ which convergenes to some point $(\hat{x}, \hat{p})$ as $j \rightarrow \infty$. Further, we see by $(52)$ that

$$
\lim _{n \rightarrow \infty}\left(x^{(n)}-x^{(n-1)}\right)=0, \quad \lim _{n \rightarrow \infty}\left(p^{(n)}-p^{(n-1)}\right)=0 .
$$

Consequently,

$$
\lim _{j \rightarrow \infty}\left(x^{\left(n_{j}-1\right)}-\hat{x}\right)=0, \quad \lim _{j \rightarrow \infty}\left(p^{\left(n_{j}-1\right)}-\hat{p}\right)=0
$$

holds true. Let $T$ denote the iteration operator of the PDHGMp cycles, i.e., $T\left(x^{(r)}, p^{(r)}\right)=$ $\left(x^{(r+1)}, p^{(r+1)}\right)$. Since $T$ is the concatenation of affine operators and proximation operators, it is continuous. Now we have that $T\left(x^{\left(n_{j}-1\right)}, p^{\left(n_{j}-1\right)}\right)=\left(x^{\left(n_{j}\right)}, p^{\left(n_{j}\right)}\right)$ and taking the limits for $j \rightarrow \infty$ we see that $T(\hat{x}, \hat{p})=(\hat{x}, \hat{p})$ so that $(\hat{x}, \hat{p})$ is a fixed point of the iteration and thus a saddle point of $L$. Using this particular saddle point in (51) and summing up from $r=n_{j}$ to $N-1, N>n_{j}$ we obtain

$$
\begin{aligned}
& \frac{1}{2 \tau}\left\|\hat{x}-x^{\left(n_{j}\right)}\right\|_{2}^{2}+\frac{1}{2 \sigma}\left\|\hat{p}-p^{\left(n_{j}\right)}\right\|_{2}^{2} \\
\geq & \frac{1}{2 \tau}\left\|\hat{x}-x^{(N)}\right\|_{2}^{2}+\frac{1}{2 \sigma}\left\|\hat{p}-p^{(N)}\right\|_{2}^{2} \\
& +\left(1-\|A\|_{2} \sqrt{\sigma \tau}\right)\left(\frac{1}{2 \tau} \sum_{r=n_{j}}^{N-1}\left\|x^{(r+1)}-x^{(r)}\right\|_{2}^{2}+\frac{1}{2 \sigma} \sum_{r=n_{j}+1}^{N-1}\left\|p^{(r)}-p^{(r-1)}\right\|^{2}\right) \\
& +\frac{1}{2 \sigma}\left\|p^{(N)}-p^{(N-1)}\right\|^{2}-\frac{\|A\|_{2} \sqrt{\sigma \tau}}{2 \sigma}\left\|p^{\left(n_{j}\right)}-p^{\left(n_{j}-1\right)}\right\|_{2}^{2} \\
& +\left\langle p^{(N)}-p^{(N-1)}, A\left(\hat{x}-x^{(N)}\right)\right\rangle-\left\langle p^{\left(n_{j}\right)}-p^{\left(n_{j}-1\right)}, A\left(\hat{x}-x^{\left(n_{j}\right)}\right)\right\rangle
\end{aligned}
$$

and further

$$
\begin{aligned}
& \frac{1}{2 \tau}\left\|\hat{x}-x^{\left(n_{j}\right)}\right\|_{2}^{2}+\frac{1}{2 \sigma}\left\|\hat{p}-p^{\left(n_{j}\right)}\right\|_{2}^{2} \\
\geq & \frac{1}{2 \tau}\left\|\hat{x}-x^{(N)}\right\|_{2}^{2}+\frac{1}{2 \sigma}\left\|\hat{p}-p^{(N)}\right\|_{2}^{2} \\
& +\frac{1}{2 \sigma}\left\|p^{(N)}-p^{(N-1)}\right\|_{2}^{2}-\frac{\|A\|_{2} \sqrt{\sigma \tau}}{2 \sigma}\left\|p^{\left(n_{j}\right)}-p^{\left(n_{j}-1\right)}\right\|_{2}^{2} \\
& +\left\langle p^{(N)}-p^{(N-1)}, A\left(\hat{x}-x^{(N)}\right)\right\rangle-\left\langle p^{\left(n_{j}\right)}-p^{\left(n_{j}-1\right)}, A\left(\hat{x}-x^{\left(n_{j}\right)}\right)\right\rangle
\end{aligned}
$$

For $j \rightarrow \infty$ this implies that $\left(x^{(N)}, p^{(N)}\right)$ converges also to $(\hat{x}, \hat{y})$ and we are done. 


\subsection{Proximal ADMM}

To avoid the computation of a linear system of equations in the first ADMM step (32), an alternative to the linearized ADMM offers the proximal ADMM algorithm 89, 181 that can be interpreted as a preconditioned variant of ADMM. In this variant the minimization step (32) is replaced by a proximal-like iteration based on the general proximal operator (5),

$$
x^{(r+1)}=\underset{x \in \mathbb{R}^{d}}{\operatorname{argmin}}\left\{L_{\gamma}\left(x, y^{(r)}, p^{(r)}\right)+\frac{1}{2}\left\|x-x^{(r)}\right\|_{R}^{2}\right\}
$$

with a symmetric, positive definite matrix $R \in \mathbb{R}^{d, d}$. The introduction of $R$ provides an additional flexibility to cancel out linear operators which might be difficult to invert. In addition the modified minimization problem is strictly convex inducing a unique minimizer. In the same manner the second ADMM step (33) can also be extended by a proximal term $(1 / 2)\left\|y-y^{(r)}\right\|_{S}^{2}$ with a symmetric, positive definite matrix $S \in \mathbb{R}^{m, m}$ [181]. The convergence analysis of the proximal ADMM was provided in [181] and the algorithm can be also classified as an inexact Uzawa method. A generalization, where the matrices $R$ and $S$ can non-monotonically vary in each iteration step, was analyzed in [89], additionally allowing an inexact computation of minimization problems.

In case of the PDHGMp algorithm, it was mentioned that the first updating step can be deduced by applying the inexact Uzawa algorithm to the first ADMM step. Using the proximal ADMM, it is straightforward to see that the first updating step of the PDHGMp algorithm with $\theta=1$ corresponds to 53 in case of $R=\frac{1}{\tau} I-\sigma A^{\mathrm{T}} A$ with $0<\tau<1 /\left\|\sigma A^{\mathrm{T}} A\right\|$, see [42, 66]. Further relations of the (proximal) ADMM to primal dual hybrid methods discussed above can be found in 66].

\subsection{Bregman Methods}

Bregman methods became very popular in image processing by a series papers of Osher and co-workers, see, e.g., [85, 128]. Many of these methods can be interpreted as ADMM methods and its linearized versions. In the following we briefly sketch these relations.

The PPA is a special case of the Bregman PPA. Let $\varphi: \mathbb{R}^{d} \rightarrow \mathbb{R} \cup\{+\infty\}$ by a convex function. Then the Bregman distance $D_{\varphi}^{p}: \mathbb{R}^{d} \times \mathbb{R}^{d} \rightarrow \mathbb{R}$ is given by

$$
D_{\varphi}^{p}(x, y)=\varphi(x)-\varphi(y)-\langle p, x-y\rangle
$$

with $p \in \partial \varphi(y), y \in \operatorname{dom} f$. If $\partial \varphi(y)$ contains only one element, we just write $D_{\varphi}$. If $\varphi$ is smooth, then the Bregman distance can be interpreted as substracting the first order Taylor expansion of $\varphi(x)$ at $y$.

Example 6.5. (Special Bregman Distances)

1. The Bregman distance corresponding to $\varphi(x):=\frac{1}{2}\|x\|_{2}^{2}$ is given by $D_{\varphi}(x, y)=\frac{1}{2}\|x-y\|_{2}^{2}$.

2. For the negative Shannon entropy $\varphi(x):=\left\langle 1_{d}, x \log x\right\rangle, x>0$ we obtain the (discrete)

I-divergence or generalized Kullback-Leibler entropy $D_{\varphi}(x, y)=x \log \frac{x}{y}-x+y$.

For $f \in \Gamma_{0}\left(\mathbb{R}^{d}\right)$ we consider the generalized proximal problem

$$
\underset{y \in \mathbb{R}^{d}}{\operatorname{argmin}}\left\{\frac{1}{\gamma} D_{\varphi}^{p}(x, y)+f(y)\right\}
$$


The Bregman Proximal Point Algorithm (BPPA) for solving this problem reads as follows:

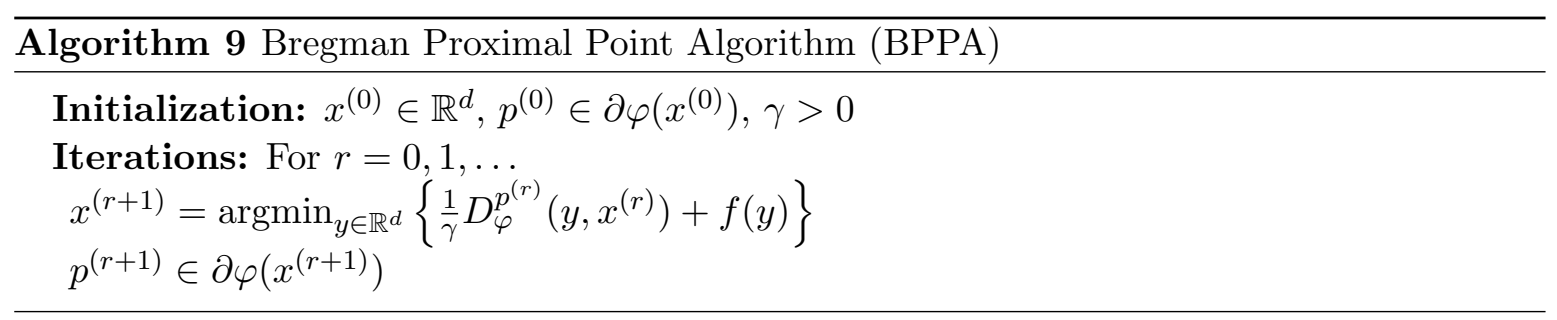

The BPPA converges for any initialization $x^{(0)}$ to a minimizer of $f$ if $f \in \Gamma_{0}\left(\mathbb{R}^{d}\right)$ attains its minimum and $\varphi$ is finite, lower semi-continuous and strictly convex. For convergence proofs we refer, e.g., to [101, 102]. We are interested again in the problem (24), i.e.,

$$
\underset{x \in \mathbb{R}^{d}, y \in \mathbb{R}^{m}}{\operatorname{argmin}}\{\Phi(x, y) \quad \text { s.t. } \quad A x=y\}, \quad \Phi(x, y):=g(x)+h(y) .
$$

We consider the BPP algorithm for the objective function $f(x, y):=\frac{1}{2}\|A x-y\|^{2}$ with the Bregman distance

$$
D_{\Phi}^{\left(p_{x}^{(r)}, p_{y}^{(r)}\right)}\left((x, y),\left(x^{(r)}, y^{(r)}\right)\right)=\Phi(x, y)-\Phi\left(x^{(r)}, y^{(r)}\right)-\left\langle p_{x}^{(r)}, x-x^{(r)}\right\rangle-\left\langle p_{y}^{(r)}, y-y^{(r)}\right\rangle,
$$

where $\left(p_{x}^{(r)}, p_{y}^{(r)}\right) \in \partial \Phi\left(x^{(r)}, y^{(r)}\right)$. This results in

$$
\begin{aligned}
\left(x^{(r+1)}, y^{(r+1)}\right) & =\underset{x \in \mathbb{R}^{d}, y \in \mathbb{R}^{m}}{\operatorname{argmin}}\left\{\frac{1}{\gamma} D_{\Phi}^{\left(p_{x}^{(r)}, p_{y}^{(r)}\right)}\left((x, y),\left(x^{(r)}, y^{(r)}\right)\right)+\frac{1}{2}\|A x-y\|^{2}\right\}, \\
p_{x}^{(r+1)} & =p_{x}^{(r)}-\gamma A^{*}\left(A x^{(r+1)}-y^{(r+1)}\right), \\
p_{y}^{(r+1)} & =p_{y}^{(r)}+\gamma\left(A x^{(r+1)}-y^{(r+1)}\right),
\end{aligned}
$$

where we have used that the first equation implies

$$
\begin{aligned}
& 0 \in \frac{1}{\gamma} \partial\left(\Phi\left(x^{(r+1)}, y^{(r+1)}\right)-\left(p_{x}^{(r)}, p_{y}^{(r)}\right)\right)+\left(A^{*}\left(A x^{(r+1)}-y^{(r+1)}\right),-\left(A x^{(r+1)}-y^{(r+1)}\right)\right), \\
& 0 \in \partial \Phi\left(x^{(r+1)}, y^{(r+1)}\right)-\left(p_{x}^{(r+1)}, p_{y}^{(r+1)}\right),
\end{aligned}
$$

so that $\left(p_{x}^{(r)}, p_{y}^{(r)}\right) \in \partial \Phi\left(x^{(r)}, y^{(r)}\right)$. From (54) and 55) we see by induction that $p_{x}^{(r)}=$ $-A^{*} p_{y}^{(r)}$. Setting $p^{(r)}=p_{y}^{(r)}$ and regarding that

$$
\begin{aligned}
& \frac{1}{\gamma} D_{\Phi}^{p^{(r)}}\left((x, y),\left(x^{(r)}, y^{(r)}\right)\right)+\frac{1}{2}\|A x-y\|_{2}^{2} \\
& =\text { const }+\frac{1}{\gamma}\left(\Phi(x, y)+\left\langle A^{*} p^{(r)}, x\right\rangle-\left\langle p^{(r)}, y\right\rangle\right)+\frac{1}{2}\|A x-y\|_{2}^{2} \\
& =\text { const }+\frac{1}{\gamma}\left(\Phi(x, y)+\frac{\gamma}{2}\left\|\frac{p^{(r)}}{\gamma}+A x-y\right\|_{2}^{2}\right)
\end{aligned}
$$

we obtain the following split Bregman method, see [85]: 


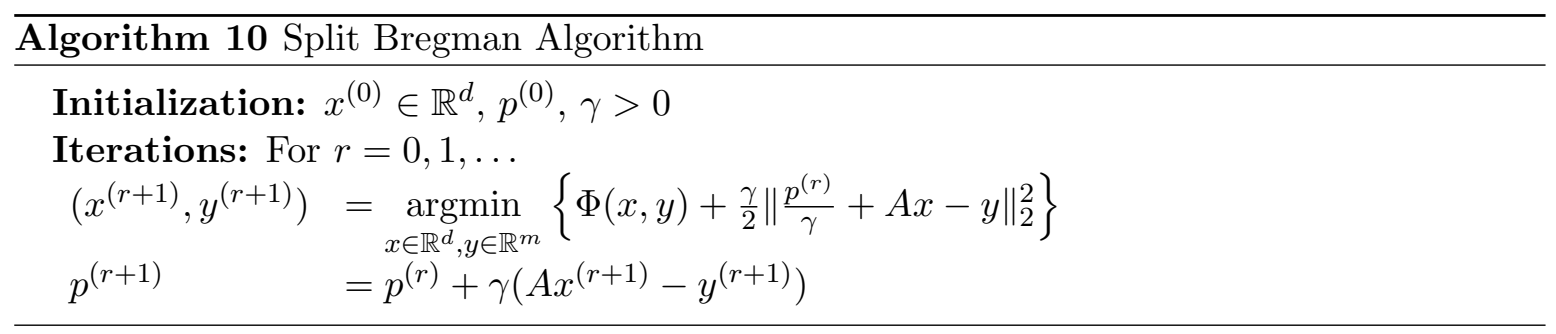

Obviously, this is exactly the form of the augmented Lagrangian method in (31).

\section{$7 \quad$ Iterative Regularization for Ill-posed Problems}

So far we have discussed the use of splitting methods for the numerical solution of well-posed variational problems, which arise in a discrete setting and in particular for the standard approach of Tikhonov-type regularization in inverse problems in imaging. The latter is based on minimizing a weighted sum of a data fidelity and a regularization functional, and can be more generally analyzed in Banach spaces, cf. [156]. However, such approaches have several disadvantages, in particular it has been shown that they lead to unnecessary bias in solutions, e.g., a contrast loss in the case of total variation regularization, cf. [129, 31]. A successful alternative to overcome this issue is iterative regularization, which directly applies iterative methods to solve the constrained variational problem

$$
\underset{x \in \mathcal{X}}{\operatorname{argmin}}\{g(x) \quad \text { s.t. } \quad A x=f\} .
$$

Here $A: \mathcal{X} \rightarrow \mathcal{Y}$ is a bounded linear operator between Banach spaces (also nonlinear versions can be considered, cf. [9, 99]) and $f$ are given data. In the well-posed case, (56) can be rephrased as the saddle-point problem

$$
\min _{x \in \mathcal{X}} \sup _{q}(g(x)-\langle q, A x-f\rangle)
$$

The major issue compared to the discrete setting is that for many prominent examples the operator $A$ does not have a closed range (and hence a discontinuous pseudo-inverse), which makes (56) ill-posed. From the optimization point of view, this raises two major issues:

- Emptyness of the constraint set: In the practically relevant case of noisy measurements one has to expect that $f$ is not in the range of $A$, i.e., the constraint cannot be satisfied exactly. Reformulated in the constrained optimization view, the standard paradigm in iterative regularization is to construct an iteration slowly increasing the functional $\mathrm{g}$ while decreasing the error in the constraint.

- Nonexistence of saddle points: Even if the data or an idealized version $A x^{*}$ to be approximated are in the range of $A$, the existence of a saddle point $\left(x^{*}, q^{*}\right)$ of 57 is not guaranteed. The optimality condition for the latter would yield

$$
A^{*} q^{*} \in \partial g\left(x^{*}\right)
$$

which is indeed an abstract smoothness condition on the subdifferential of $g$ at $x^{*}$ if $A$ and consequently $A^{*}$ are smoothing operators, it is known as source condition in the field of inverse problems, cf. [31]. 
Due to the above reasons the use of iterative methods for solving respectively approximating (56) has a different flavour than iterative methods for well-posed problems. The key idea is to employ the algorithm as an iterative regularization method, cf. [99, where appropriate stopping in dependence on the noise, i.e. a distance between $A x^{*}$ and $f$, needs to be performed in order to obtain a suitable approximation. The notion to be used is called semiconvergence, i.e., if $\delta>0$ denotes a measure for the data error (noise level) and $\hat{r}(\delta)$ is the stopping index of the iteration in dependence on $\delta$, then we look for convergence

$$
x^{(\hat{r}(\delta))} \rightarrow x^{*} \quad \text { as } \delta \rightarrow 0,
$$

in a suitable topology. The minimal ingredient in the convergence analysis is the convergence $x^{(r)} \rightarrow x^{*}$, which already needs different approaches as discussed above. For iterative methods working on primal variables one can at least use the existence of $(56)$ in this case, while real primal-dual iterations still suffer from the potential nonexistence of solutions of the saddle point problem (57).

A well-understood iterative method is the Bregman iteration

$$
x^{(r+1)} \in \underset{x \in \mathcal{X}}{\operatorname{argmin}}\left(\frac{\mu}{2}\|A x-f\|^{2}+D_{g}^{p^{(r)}}\left(x, x^{(r)}\right)\right),
$$

with $p^{(r)} \in \partial g\left(x^{(r)}\right)$, which has been analyzed as an iterative method in [129], respectively for nonlinear $A$ in [9]. Note that again with $p^{(r)}=A^{*} q^{(r)}$ the Bregman iteration is equivalent to the augmented Lagrangian method for the saddle-point problem (57). With such iterative regularization methods superior results compared to standard variational methods can be computed for inverse and imaging problems, in particular bias can be eliminated, cf. [31]. The key properties are the decrease of the data fidelity

$$
\left\|A x^{(r+1)}-f\right\|^{2} \leq\left\|A x^{(r)}-f\right\|^{2},
$$

for all $r$ and the decrease of the Bregman distance to the clean solution

$$
D_{g}^{p^{(r+1)}}\left(x^{*}, x^{(r+1)}\right) \leq D_{g}^{p^{(r)}}\left(x^{*}, x^{(r)}\right)
$$

for those $r$ such that

$$
\left\|A x^{(r)}-f\right\| \geq\left\|A x^{*}-f\right\|=\delta .
$$

Together with a more detailed analysis of the difference between consecutive Bregman distances, this can be used to prove semiconvergence results in appropriate weak topologies, cf. [129, 156]. In 9] further variants approximating the quadratic terms, such as the linearized Bregman iteration, are analyzed, however with further restrictions on $g$. For all other iterative methods discussed above, a convergence analysis in the case of ill-posed problems is completely open and appears to be a valuable task for future research. Note that in the realization of the Bregman iteration, a well-posed but complex variational problem needs to be solved in each step. By additional operator splitting in an iterative regularization method one could dramatically reduce the computational effort.

If the source condition is satisfied, i.e. if there exists a saddle-point $\left(x^{*}, q^{*}\right)$, one can further exploit the decrease of dual distances

$$
\left\|q^{(r+1)}-q^{*}\right\| \leq\left\|q^{(r)}-q^{*}\right\|
$$

to obtain a quantitative estimate on the convergence speed, we refer to [30, 32] for a further discussion. 


\section{Applications}

So far we have focused on technical aspects of first order algorithms whose (further) development has been heavily forced by practical applications. In this section we give a rough overview of the use of first order algorithms in practice. We start with applications from classical imaging tasks such as computer vision and image analysis and proceed to applications in natural and life sciences. From the area of biomedical imaging, we will present the Positron Emission Tomography (PET) and Spectral X-ray $C T$ in more detail and show some results reconstructed with first order algorithms.

At the beginning it is worth to emphasize that many algorithms based on proximal operators such as proximal point algorithm, proximal forward-backward splitting, ADMM, or DouglasRachford splitting have been introduced in the 1970s, cf. [78, 109, 139, 140]. However these algorithms have found a broad application in the last two decades, mainly caused by the technological progress. Due to the ability of distributed convex optimization with ADMM related algorithms, these algorithms seem to be qualified for 'big data' analysis and large-scale applications in applied statistics and machine learning, e.g., in areas as artificial intelligence, internet applications, computational biology and medicine, finance, network analysis, or logistics [27, 132]. Another boost for the popularity of first order splitting algorithms was the increasing use of sparsity-promoting regularizers based on $\ell_{1}$ - or $L_{1}$-type penalties [85, 144], in particular in combination with inverse problems considering non-linear image formation models [9, 165] and/or statistically derived (inverse) problems [31]. The latter problems lead to non-quadratic fidelity terms which result from the non-Gaussian structure of the noise model. The overview given in the following mainly concentrates on the latter mentioned applications.

The most classical application of first order splitting algorithms is image analysis such as denoising, where these methods were originally pushed by the Rudin, Osher, and Fatemi (ROF) model [144]. This model and its variants are frequently used as prototypes for total variation methods in imaging to illustrate the applicability of proposed algorithms in case of non-smooth cost functions, cf. [42, 65, 66, 85, 128, 157, 182. Since the standard $L_{2}$ fidelity term is not appropriate for non-Gaussian noise models, modifications of the ROF problem have been considered in the past and were solved using splitting algorithms to denoise images perturbed by non-Gaussian noise, cf. [19, 42, 71, 146, 161]. Due to the close relation of total variation techniques to image segmentation [31, 133], first order algorithms have been also applied in this field of applications (cf. [41, 42, 84, 133]). Other image analysis tasks where proximal based algorithms have been applied successfully are deblurring and zooming (cf. [23, 42, 66, 159]), inpainting [42], stereo and motion estimation [44, 42, 179], and segmentation [10, 108, 133, 100].

Due to increasing noise level in modern biomedical applications, the requirement on statistical image reconstruction methods has been risen recently and the proximal methods have found access to many applied areas of biomedical imaging. Among the enormous amount of applications from the last two decades, we only give the following selection and further links to the literature:

- X-ray $C T$ : Recently statistical image reconstruction methods have received increasing attention in X-ray CT due to increasing noise level encountered in modern CT applications such as sparse/limited-view CT and low-dose imaging, cf., e.g., [166, 171, 172], or $\mathrm{K}$-edge imaging where the concentrations of K-edge materials are inherently low, see, 
e.g., 152, 151, 153. In particular, first order splitting methods have received strong attention due to the ability to handle non-standard noise models and sparsity-promoting regularizers efficiently. Beside the classical fan-beam and cone-beam X-ray CT (see, e.g., [4, 43, 48, 98, 123, 138, 160, 166]), the algorithms have also found applications in emerging techniques such as spectral CT, see Section 8.2 and [80, 148, 178] or phase contrast CT [56, 124, 177].

- Magnetic resonance imaging (MRI): Image reconstruction in MRI is mainly achieved by inverting the Fourier transform which can be performed efficiently and robustly if a sufficient number of Fourier coefficients is measured. However, this is not the case in special applications such as fast MRI protocols, cf., e.g., [110, 135], where the Fourier space is undersampled so that the Nyquist criterion is violated and Fourier reconstructions exhibit aliasing artifacts. Thus, compressed sensing theory have found the way into MRI by exploiting sparsity-promoting variational approaches, see, e.g., [15, 97, 111, 137]. Furthermore, in advanced MRI applications such as velocity-encoded MRI or diffusion MRI, the measurements can be modeled more accurately by nonlinear operators and splitting algorithms provide the ability to handle the increased reconstruction complexity efficiently [165].

- Emission tomography: Emission tomography techniques used in nuclear medicine such as positron emission tomography (PET) and single photon emission computed tomography (SPECT) [176] are classical examples for inverse problems in biomedical imaging where statistical modeling of the reconstruction problem is essential due to Poisson statistics of the data. In addition, in cases where short time or low tracer dose measurements are available (e.g., using cardiac and/or respiratory gating 34]) or tracer with a short radioactive half-life are used (e.g., radioactive water $\mathrm{H}_{2}{ }^{15} \mathrm{O}$ [150]), the measurements suffer from inherently high noise level and thus a variety of first order splitting algorithms has been utilized in emission tomography, see, e.g., 4, 16, 115, 116, 136, 146].

- Optical microscopy: In modern light microscopy techniques such as stimulated emission depletion (STED) or 4Pi-confocal fluorescence microscopy [93, 94, resolutions beyond the diffraction barrier can be achieved allowing imaging at nanoscales. However, by reaching the diffraction limit of light, measurements suffer from blurring effects and Poisson noise with low photon count rates [61, 155, in particular in live imaging and in high resolution imaging at nanoscopic scales. Thus, regularized (blind) deconvolution addressing appropriate Poisson noise is quite beneficial and proximal algorithms have been applied to achieve this goal, cf., e.g., [30, 76, 146, 159].

- Other modalities: It is quite natural that first order splitting algorithms have found a broad usage in biomedical imaging, in particular in such applications where the measurements are highly perturbed by noise and thus regularization with probably a proper statistical modeling are essential as, e.g., in optical tomography [1, 75], medical ultrasound imaging [147], hybrid photo-/optoacoustic tomography [79, 173], or electron tomography [86].

\subsection{Positron Emission Tomography (PET)}

PET is a biomedical imaging technique visualizing biochemical and physiological processes such as glucose metabolism, blood flow, or receptor concentrations, see, e.g., [176]. This 
modality is mainly applied in nuclear medicine and the data acquisition is based on weak radioactively marked pharmaceuticals (so-called tracers), which are injected into the blood circulation. Then bindings dependent on the choice of the tracer to the molecules are studied. Since the used markers are radio-isotopes, they decay by emitting a positron which annihilates almost immediately with an electron. The resulting emission of two photons is detected and, due to the radioactive decay, the measured data can be modeled as an inhomogeneous Poisson process with a mean given by the X-ray transform of the spatial tracer distribution (cf., e.g., [117, 167]). Note that, up to notation, the X-ray transform coincides with the more popular Radon transform in the two dimensional case [117]. Thus, the underlying reconstruction problem can be modeled as

$$
\sum_{m=1}^{M}\left((K u)_{m}-f_{m} \log \left((K u)_{m}\right)\right)+\alpha R(u) \rightarrow \min _{u \geq 0}, \quad \alpha>0,
$$

where $M$ is the number of measurements, $f$ are the given data, and $K$ is the system matrix which describes the full properties of the PET data acquisition.

To solve (64), algorithms discussed above can be applied and several of them have been already studied for PET recently. In the following, we will give a (certainly incomplete) performance discussion of different first order splitting algorithms on synthetic PET data and highlight the strengths and weaknesses of them which could be carried over to many other imaging applications. For the study below, the total variation (TV) was applied as regularization energy $R$ in (64) and the following algorithms and parameter settings were used for the performance evaluation:

- $\boldsymbol{F B}-\boldsymbol{E M}-\boldsymbol{T} \boldsymbol{V}$ : The FB-EM-TV algorithm [146] represents an instance of the proximal forward-backward (FB) splitting algorithm discussed in Section 5.2 using a variable metric strategy (22). The preconditioned matrices $Q^{(r)}$ in $(22)$ are chosen in a way that the gradient descent step corresponds to an expectation-maximization (EM) reconstruction step. The EM algorithm is a classically applied (iterative) reconstruction method in emission tomography [117, 167]. The TV proximal problem was solved by an adopted variant of the modified Arrow-Hurwicz method proposed in 42 since it was shown to be the most efficient method for TV penalized weighted least-squares denoising problems in [145]. Furthermore, a warm starting strategy was used to initialize the dual variables within the TV proximal problem and the inner iteration sequence was stopped if the relative error of primal and dual optimality conditions was below an error tolerance $\delta$, i.e., using the notations from [42, if

$$
\max \left\{d^{(r)}, p^{(r)}\right\} \leq \delta
$$

with

$$
\begin{aligned}
& d^{(r)}=\left\|\left(y^{(r)}-y^{(r-1)}\right) / \sigma_{r-1}+\nabla\left(x^{(r)}-x^{(r-1)}\right)\right\| /\left\|\nabla x^{(r)}\right\|, \\
& p^{(r)}=\left\|x^{(r)}-x^{(r-1)}\right\| /\left\|x^{(r)}\right\| .
\end{aligned}
$$

The damping parameter $\eta^{(r)}$ in $(22)$ was set to $\eta^{(r)}=1$ as indicated in [146].

- FB-EM-TV-Nes83: A modified version of FB-EM-TV described above using the acceleration strategy proposed by Nesterov in [121]. This modification can be seen as a 
variant of FISTA [13] with a variable metric strategy (22). Here, $\eta^{(r)}$ in $(22)$ was chosen fixed (i.e. $\eta^{(r)}=\eta$ ) but has to be adopted to the pre-defined inner accuracy threshold $\delta$ (65) to guarantee the convergence of the algorithm and it was to be done manually.

- $\boldsymbol{C P}-\boldsymbol{E}$ : The fully explicit variant of the Chambolle-Pock's primal-dual algorithm 42 ] (cf. Section 6.3) studied for PET reconstruction problems in [3] (see CP2TV in [3]). The dual step size $\sigma$ was set manually and the primal one corresponding to [42] as $\tau \sigma\left(\|\nabla\|^{2}+\|K\|^{2}\right)=1$, where $\|K\|$ was pre-estimated using the Power method.

- Precond-CP-E: The CP-E algorithm described above but using the diagonal preconditioning strategy proposed in [41] with $\alpha=1$ in [41, Lemma 2].

- $\boldsymbol{C P}$-SI: The semi-implicit variant of the Chambolle-Pock's primal-dual algorithm [42] (cf. Section 6.3) studied for PET reconstruction problems in [3] (see CP1TV in [3]). The difference to CP-E is that a TV proximal problem has to be solved in each iteration step. This was performed as in case of FB-EM-TV method. Furthermore, the dual step size $\sigma$ was set manually and the primal one corresponding to [42] as $\tau \sigma\|K\|^{2}=1$, where $\|K\|$ was pre-estimated using the Power method.

- Precond-CP-SI: The CP-SI algorithm described above but using the diagonal preconditioning strategy proposed in [41] with $\alpha=1$ in [41, Lemma 2].

- PIDSplit+: An ADMM based algorithm (cf. Section 6.2 that has been discussed for Poisson deblurring problems of the form (64) in [159. However, in case of PET reconstruction problems, the solution of a linear system of equations of the form

$$
\left(I+K^{\mathrm{T}} K+\nabla^{\mathrm{T}} \nabla\right) u^{(r+1)}=z^{(r)}
$$

has to be computed in a different way in contrast to deblurring problems. This was done by running two preconditioned conjugate gradient (PCG) iterations with warm starting and cone filter preconditioning whose effectiveness has been validated in [138] for X-ray CT reconstruction problems. The cone filter was constructed as described in [68, 69] and diagonalized by the discrete cosine transform (DCT-II) supposing Neumann boundary conditions. The PIDSplit+ algorithm described above can be accomplished by a strategy of adaptive augmented Lagrangian parameters $\gamma$ in 26 as proposed for the PIDSplit+ algorithm in [27, 162]. The motivation behind this strategy is to mitigate the performance dependency on the initial chosen fixed parameter that may strongly influence the speed of convergence of ADMM based algorithms.

All algorithms were implemented in MATLAB and executed on a machine with 4 CPU cores, each $2.83 \mathrm{GHz}$, and $7.73 \mathrm{~GB}$ physical memory, running a 64 bit Linux system and MATLAB 2013b. The built-in multi-threading in MATLAB was disabled such that all computations were limited to a single thread. The algorithms were evaluated on a simple object (image size $256 \times 256)$ and the synthetic 2D PET measurements were obtained via a Monte-Carlo simulation with 257 radial and 256 angular samples, using one million simulated events (see Figure 2). Due to sustainable image and measurement dimensions, the system matrix $K$ was pre-computed for all reconstruction runs. To evaluate the performance of algorithms described above, the following procedure was applied. First, since $K$ is injective and thus an unique minimizer of (64) is guaranteed [146], we can run a well performing method for a very 

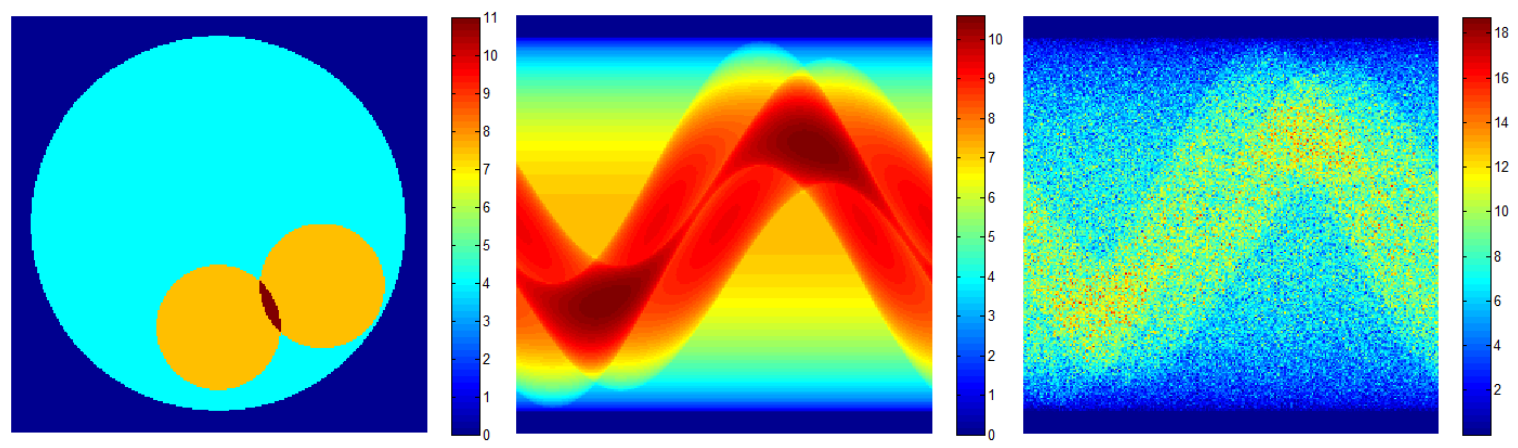

Figure 2: Synthetic 2D PET data. Left: Exact object. Middle: Exact Radon data. Right: Simulated PET measurements via a Monte-Carlo simulation using one million events.
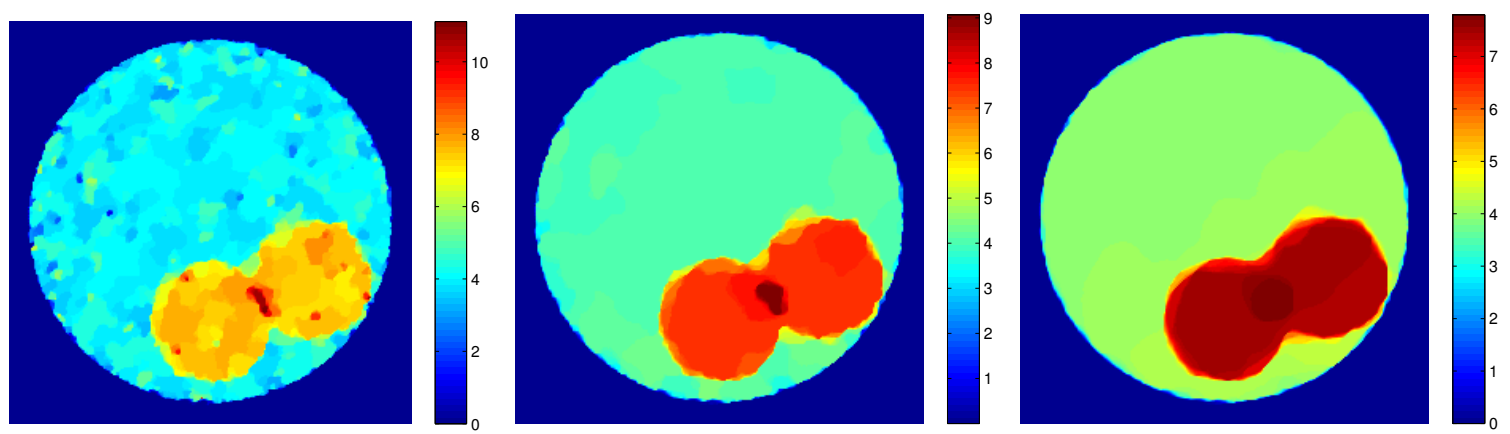

Figure 3: The "ground truth" solutions for regularization parameter values $\alpha=0.04$ (left), $\alpha=0.08$ (middle), and $\alpha=0.20$ (right).

long time to compute a "ground truth" solution $u_{\alpha}^{*}$ for a fixed $\alpha$. To this end, we have run the Precond-CP-E algorithm for 100,000 iterations for following reasons: (1) all iteration steps can be solved exactly such that the solution cannot be influenced by inexact computations (see discussion below); (2) due to preconditioning strategy, no free parameters are available those may influence the speed of convergence negatively such that $u_{\alpha}^{*}$ is expected to be of high accuracy after 100,000 iterations. Having $u_{\alpha}^{*}$, each algorithm was applied until the relative error

$$
\left\|u_{\alpha}^{(r)}-u_{\alpha}^{*}\right\| /\left\|u_{\alpha}^{*}\right\|
$$

was below a pre-defined threshold $\epsilon$ (or a maximum number of iterations adopted for each algorithm individually was reached). The "ground truth" solutions for three different values of $\alpha$ are shown in Figure 3 .

Figures 4-8 show the performance evaluation of algorithms plotting the propagation of the relative error (67) in dependency on the number of iterations and CPU time in seconds. Since all algorithms have a specific set of unspecified parameters, different values of them are plotted to give a representative overall impression. The reason for showing the performance both in dependency on the number of iterations and CPU time is twofold: (1) in the presented case where the PET system matrix $K$ is pre-computed and thus available explicitly, the evaluation of forward and backward projections is nearly negligible and TV relevant computations have the most contribution to the run time such that the CPU time will be a good indicator for 

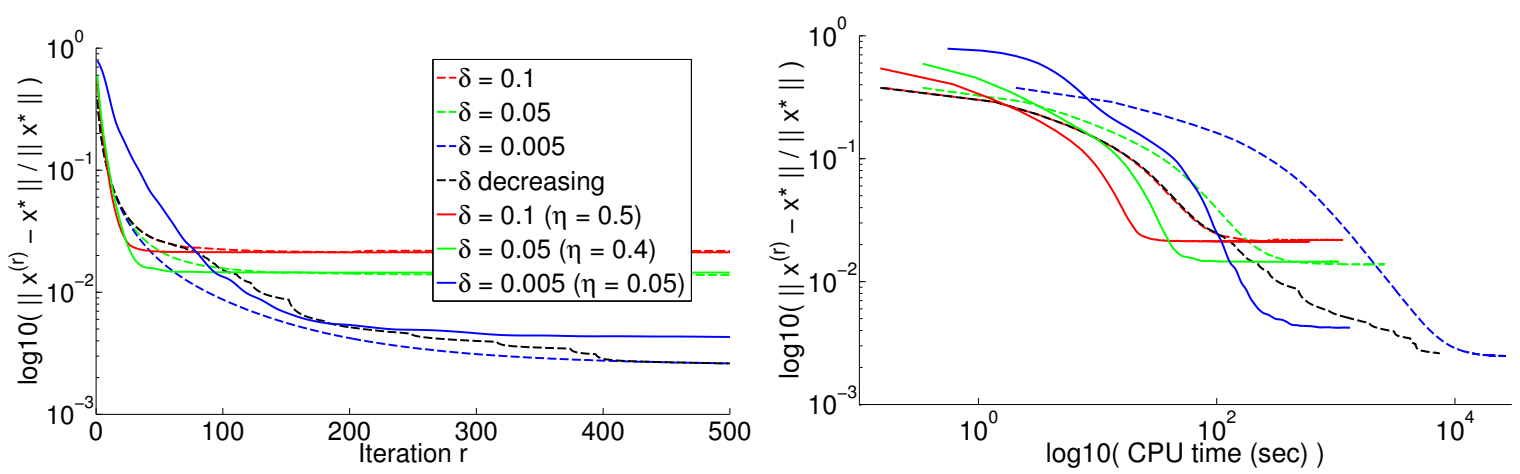

Figure 4: Performance of FB-EM-TV (dashed lines) and FB-EM-TV-Nes83 (solid lines) for different accuracy thresholds $\delta(65)$ within the TV proximal step. Evaluation of relative error (67) is shown as a function of the number of iterations (left) and CPU time in seconds (right).

algorithm's performance; (2) in practically relevant cases where the forward and backward projections have to be computed in each iteration step implicitly and in general are computationally consuming, the number of iterations and thus the number of projection evaluations will be the crucial factor for algorithm's efficiency. In the following, we individually discuss the behavior of algorithms observed for the regularization parameter $\alpha=0.08$ (64) with the "ground truth" solution shown in Figure 3 .

- FB-EM-TV(-Nes83): The evaluation of FB-EM-TV based algorithms is shown in Figure 4. The major observation for any $\delta$ in (65) is that the inexact computations of TV proximal problems lead to a restrictive approximation of the "ground truth" solution where the approximation accuracy stagnates after a specific number of iterations depending on $\delta$. In addition, it can also be observed that the relative error (67) becomes better with more accurate TV proximal solutions (i.e. smaller $\delta$ ) indicating that a decreasing sequence $\delta^{(r)}$ should be used to converge against the solution of (64) (see, e.g., 154, 168 for convergence analysis of inexact proximal gradient algorithms). However, as indicated in [112] and is shown in Figure 4, the choice of $\delta$ provides a trade-off between the approximation accuracy and computational cost such that the convergence rates proved in [154, 168] might be computationally not optimal. Another observation concerns the accelerated modification FB-EM-TV-Nes83. In Figure 4 we can observe that the performance of FB-EM-TV can actually be improved by FB-EM-TV-Nes83 regarding the number of iterations but only for smaller values of $\delta$. One reason might be that using FB-EM-TV-Nes83 we have seen in our experiments that the gradient descent parameter $0<\eta \leq 1$ [146] in (22) has to be chosen smaller with increased TV proximal accuracy (i.e. smaller $\delta$ ). Since in such cases the effective regularization parameter value in each TV proximal problem is $\eta \alpha$, a decreasing $\eta$ will result in poorer denoising properties increasing the inexactness of TV proximal operator. Recently, an (accelerated) inexact variable metric proximal gradient method was analyzed in [49] providing a theoretical view on such a type of methods.

- (Precond-) CP-E: In Figure 5, the algorithms CP-E and Precond-CP-E are evaluated. In contrast to FB-EM-TV-(Nes83), the approximated solution cannot be influenced by inexact computations such that a decaying behavior of relative error can be observed. The single parameter that affects the convergence rate is the dual steplength $\sigma$ and 

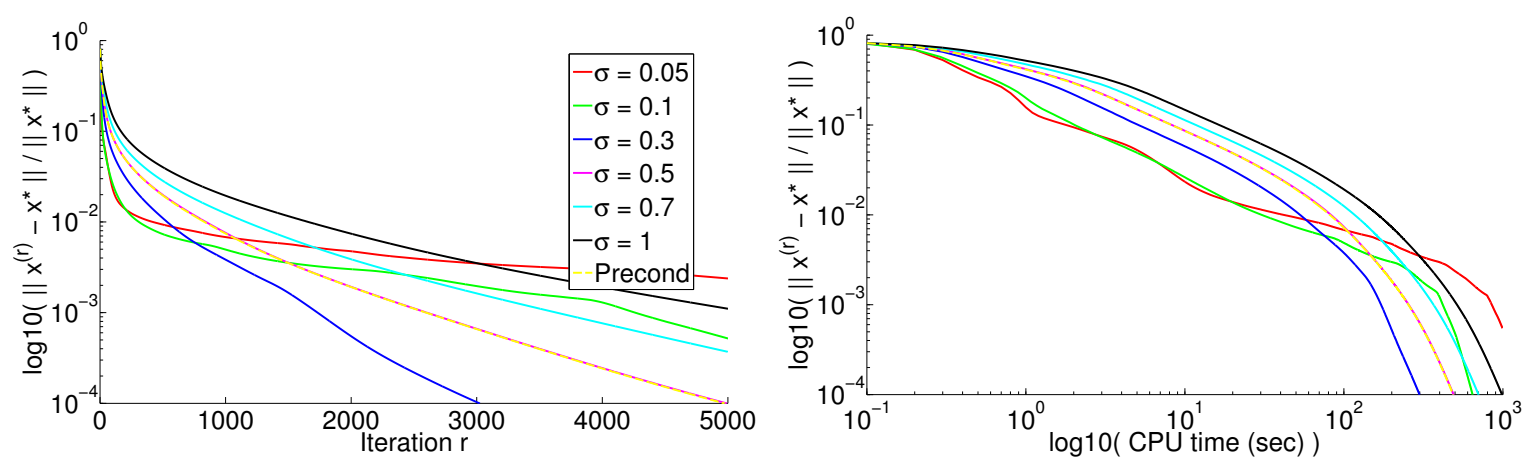

(a) Evaluation of relative error for fixed dual step sizes $\sigma$.
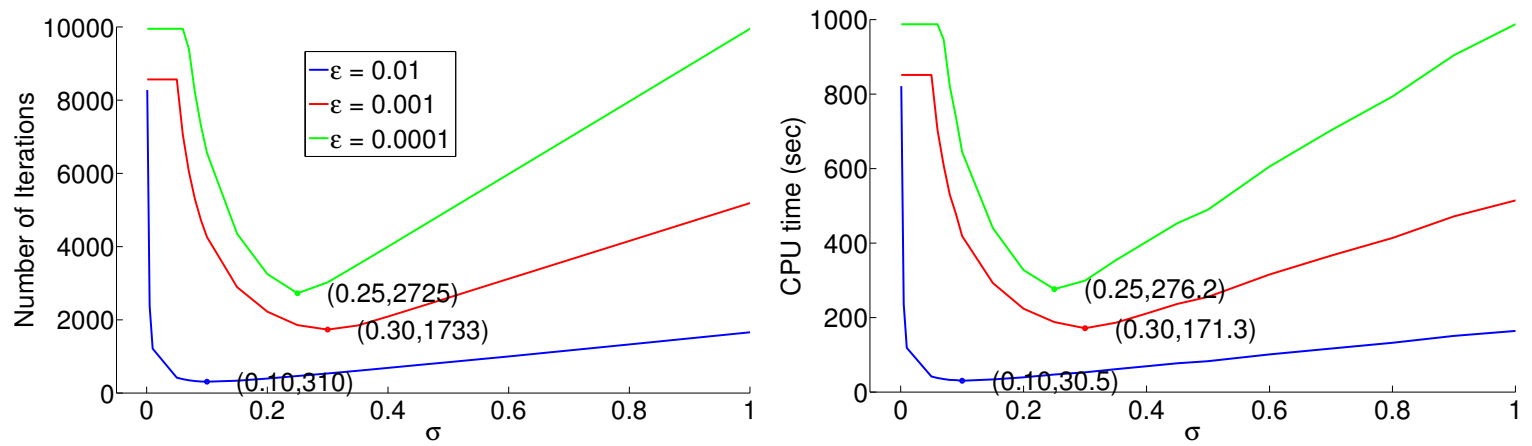

(b) Performance to get the relative error below the threshold $\epsilon$ as a function of dual step size $\sigma$.

Figure 5: Performance of Precond-CP-E (dashed lines in (a) and CP-E (solid lines) for different dual step sizes $\sigma$. (a) Evaluation of relative error as a function of the number of iterations (left) and CPU time in seconds (right). (b) Required number of iterations (left) and CPU time (right) to get the relative error below a pre-defined threshold $\epsilon$ as a function of $\sigma$.

we observe in Figure 5 a that some values yield a fast initial convergence (see, e.g., $\sigma=0.05$ and $\sigma=0.1$ ), but are less suited to achieve fast asymptotic convergence and vice versa (see, e.g., $\sigma=0.3$ and $\sigma=0.5$ ). However, the plots in Figure 5b indicate that $\sigma \in[0.2,0.3]$ may provide an acceptable trade-off between initial and asymptotic convergence in terms of the number of iterations and CPU time. Regarding the latter mentioned aspect we note that in case of CP-E the more natural setting of $\sigma$ would be $\sigma=\sqrt{\|\nabla\|^{2}+\|K\|^{2}}$ what is approximately 0.29 in our experiments providing acceptable trade-off between initial and asymptotic convergence. Finally, no acceleration was observed in case of Precond-CP-E algorithm due to the regular structure of linear operators $\nabla$ and $K$ in our experiments such that the performance is comparable to CP-E with $\sigma=0.5$ (see Figure 5a).

- (Precond-)CP-SI: In Figures 6 and 7, the evaluation of CP-SI and Precond-CP-SI is presented. Since a TV proximal operator has to be approximated in each iteration step, the same observations can be made as in case of FB-EM-TV that depending on $\delta$ the relative error stagnates after a specific number of iterations and that the choice of $\delta$ provides a trade-off between approximation accuracy and computational time (see Figure 6 for Precond-CP-SI). In addition, since the performance of CP-SI not only 

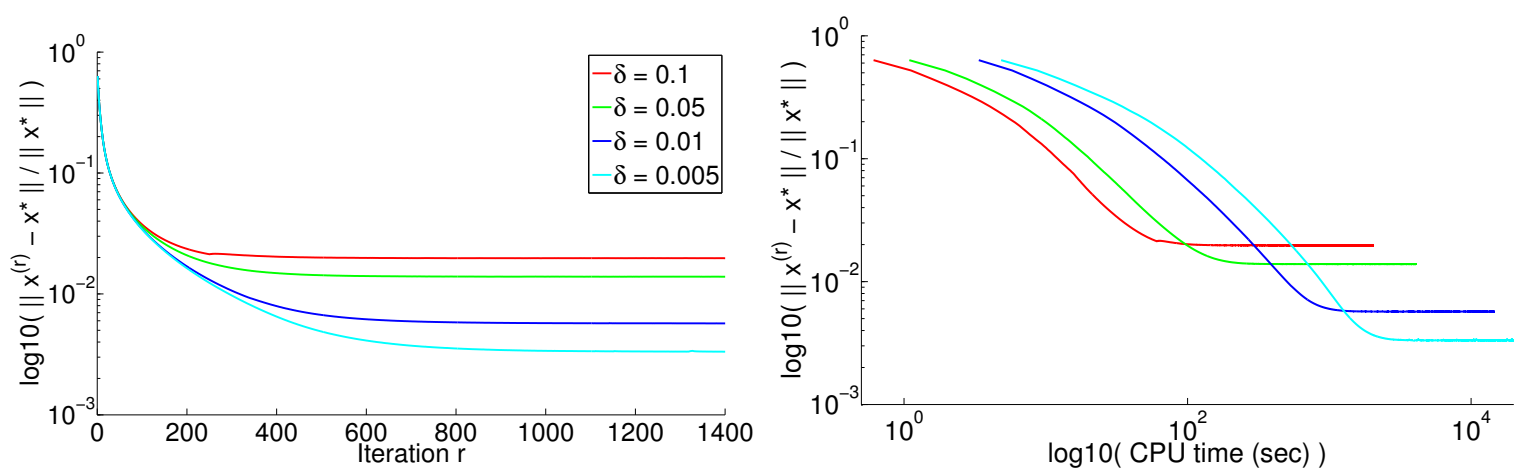

Figure 6: Performance of Precond-CP-SI for different accuracy thresholds $\delta$ (65) within the TV proximal step. Evaluation of relative error as a function of number of iterations (left) and CPU time in seconds (right).

depends on $\delta$ but also on the dual steplength $\sigma$, the evaluation of CP-SI for different values of $\sigma$ and two stopping values $\delta$ is shown in Figure 7. The main observation is that for smaller $\sigma$ a better initial convergence can be achieved in terms of the number of iterations but results in less efficient performance regarding the CPU time. The reason is that the effective regularization parameter within the TV proximal problem is $\tau \alpha$ (see (22)) with $\tau=\left(\sigma\|K\|^{2}\right)^{-1}$ and a decreasing $\sigma$ leads to an increasing TV denoising effort. Thus, in practically relevant cases, $\sigma$ should be chosen optimally in a way balancing the required number of iterations and TV proximal computation.

- PIDSplit+: In Figure 8 the performance of PIDSplit+ is shown. It is well evaluated that the convergence of ADMM based algorithms is strongly dependent on the augmented Lagrangian parameter $\gamma(26)$ and that some values yield a fast initial convergence but are less suited to achieve a fast asymptotic convergence and vice versa. This behavior can also be observed in Figure 8 (see $\gamma=30$ in upper row).

Finally, to get a feeling how the algorithms perform against each other, the required CPU time and number of projection evaluations to get the relative error (67) below a pre-defined threshold are shown in Table 1 for two different values of $\epsilon$. The following observations can be made:

- The FB-EM-TV based algorithms are competitive in terms of required number of projection evaluations but have a higher CPU time due to the computation of TV proximal operators, in particular the CPU time strongly grows with decreasing $\epsilon$ since TV proximal problems have to be approximated with increased accuracy. However, in our experiments, a fixed $\delta$ was used in each TV denoising step and thus the performance can be improved utilizing the fact that a rough accuracy is sufficient at the beginning of the iteration sequence without influencing the performance regarding the number of projector evaluations negatively (cf. Figure 4). Thus, a proper strategy to iteratively decrease $\delta$ in (65) can strongly improve the performance of FB-EM-TV based algorithms.

- The CP-E algorithm is optimal in our experiments in terms of CPU time since the TV regularization is computed by the shrinkage operator and thus is simply to evaluate. However, this algorithm needs almost the highest number of projection evaluations 

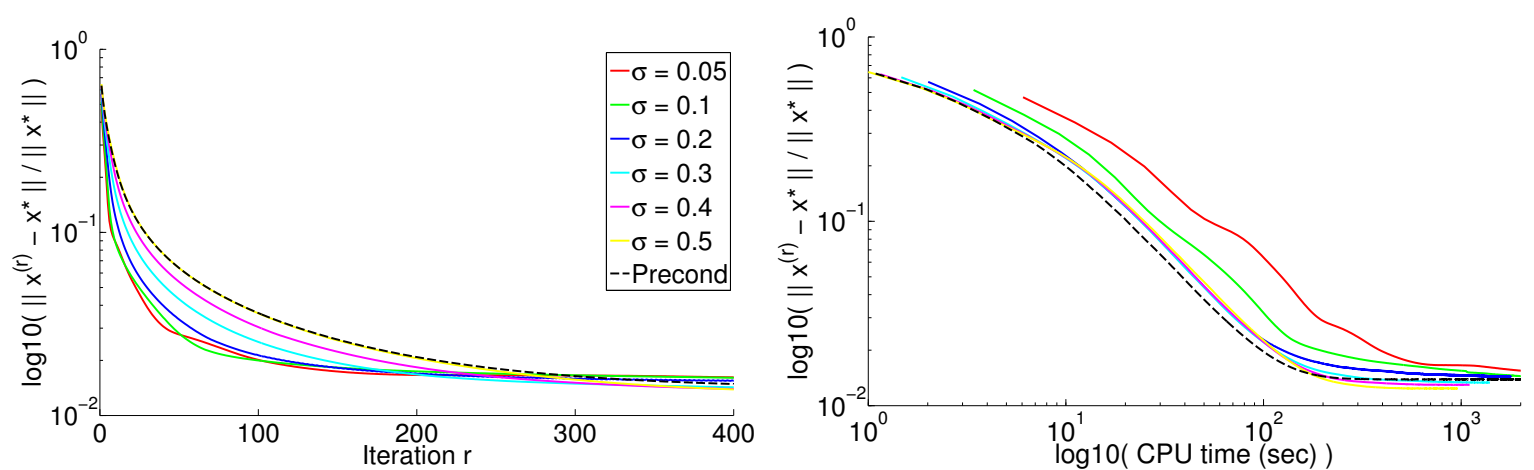

(a) Performance with TV proximal accuracy $\delta=0.05(65)$.
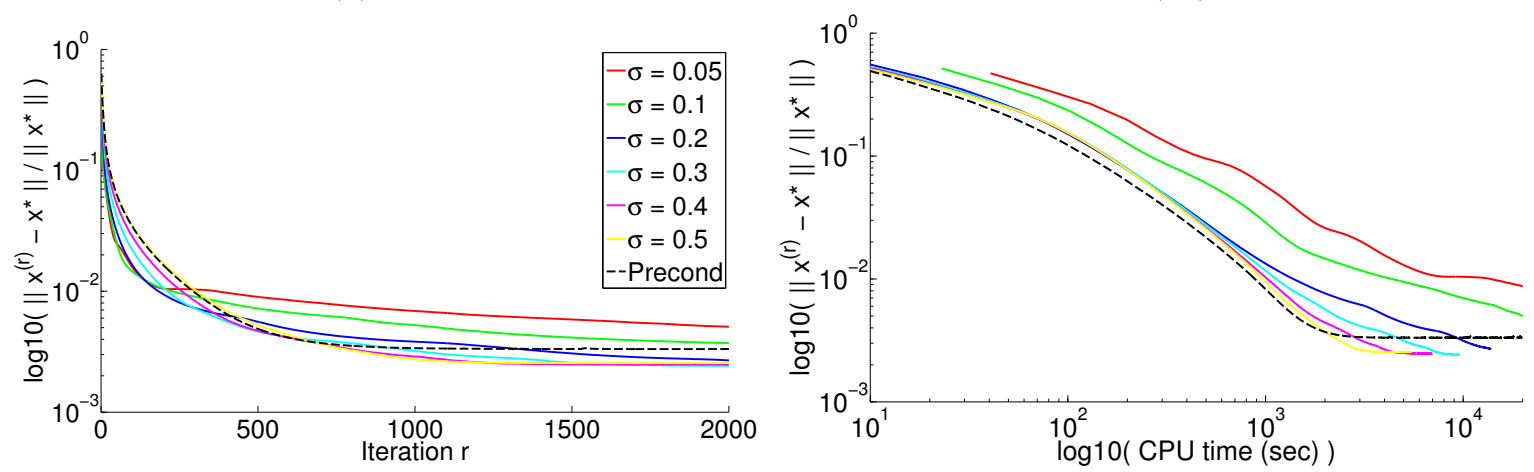

(b) Performance with TV proximal accuracy $\delta=0.00565$.

Figure 7: Performance of Precond-CP-SI (dashed lines) and CP-SI (solid lines) for different dual step sizes $\sigma$. Evaluation of relative error as a function of number of iterations (left) and CPU time in seconds (right) for accuracy thresholds $\delta=0.05$ (a) and $\delta=0.005$ (b) within the TV proximal problem.
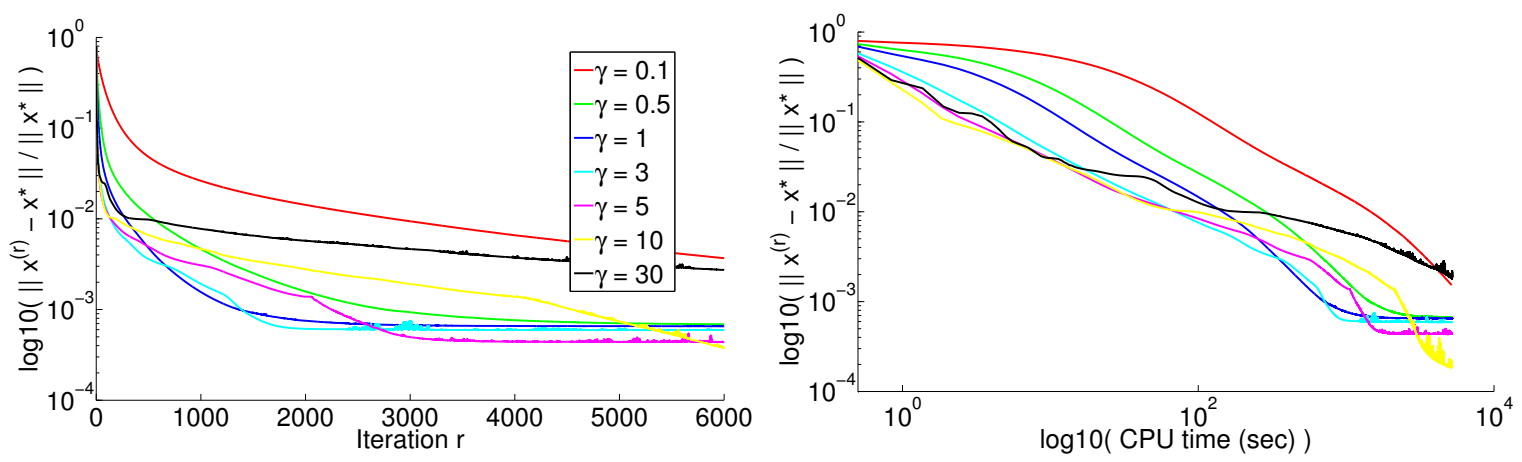

Figure 8: Performance of PIDSplit+ for fixed augmented Lagrangian penalty parameters $\gamma$ (26). Evaluation of relative error as a function of number of iterations (left) and CPU time in seconds (right). 
Table 1: Performance evaluation of algorithms described above for $\alpha=0.08$ (see $u_{\alpha}^{*}$ in Figure 3 (middle)). The table displays the CPU time in seconds and required number of forward and backward projector evaluations $\left(K / K^{\mathrm{T}}\right)$ to get the relative error (67) below the error tolerance $\epsilon$. For each algorithm the best performance regarding the CPU time and $K / K^{\mathrm{T}}$ evaluations are shown where $\|$ means that the value coincides with the value directly above.

\begin{tabular}{|c|c|c|c|c|c|}
\hline & & \multicolumn{2}{|c|}{$\epsilon=0.05$} & \multicolumn{2}{|c|}{$\epsilon=0.005$} \\
\hline & & $K / K^{\mathrm{T}}$ & $\mathrm{CPU}$ & $K / K^{\mathrm{T}}$ & $\mathrm{CPU}$ \\
\hline FB-EM-TV & (best $K / K^{\mathrm{T}}$ ) & 20 & 40.55 & 168 & 4999.71 \\
\hline 11 & (best CPU) & 11 & $\|$ & 230 & 3415.74 \\
\hline FB-EM-TV-Nes 83 & (best $K / K^{\mathrm{T}}$ ) & 15 & 14.68 & 231 & 308.74 \\
\hline$\|$ & (best CPU) & 11 & $\|$ & 11 & 11 \\
\hline CP-E & (best $K / K^{\mathrm{T}}$ ) & 48 & 4.79 & 696 & 69.86 \\
\hline 11 & (best CPU) & 11 & 11 & 11 & 11 \\
\hline CP-SI & (best $K / K^{\mathrm{T}}$ ) & 22 & 198.07 & 456 & 1427.71 \\
\hline 11 & (best CPU) & 25 & 23.73 & 780 & 1284.56 \\
\hline PIDSplit+ & (best $K / K^{\mathrm{T}}$ ) & 30 & 7.51 & 698 & 179.77 \\
\hline 11 & (best CPU) & 11 & 11 & 11 & 11 \\
\hline
\end{tabular}

that will result in a slow algorithm in practically relevant cases where the projector evaluations are highly computationally expansive.

- The PIDSplit+ algorithm is slightly poorer in terms of CPU time than CP-E but required a smaller number of projector evaluations. However, we remind that this performance may probably be improved since two PCG iterations were used in our experiments and thus two forward and backward projector evaluations are required in each iteration step of PIDSplit+ method. Thus, if only one PCG step is used, the CPU time and number of projector evaluations can be decreased leading to a better performing algorithm. However, in the latter case, the total number of iteration steps might be increased since a poorer approximation of (66) will be performed if only one PCG step is used. Another opportunity to improve the performance of PIDSplit+ algorithm is to use the proximal ADMM strategy described in Section 6.4, namely, to remove $K^{\mathrm{T}} K$ from (66). That will result in only a single evaluation of forward and backward projectors in each iteration step but may lead to an increased number of total number of algorithm iterations.

Finally, to study the algorithm's stability regarding the choice of regularization parameter $\alpha$, we have run the algorithms for two additional values of $\alpha$ using the parameters shown the best performance in Table 1. The additional penalty parameters include a slightly undersmoothed and over-smoothed result respectively as shown in Figure 3 and the evaluation results are shown in Tables 2 and 3 . In the following we describe the major observations:

- The FB-EM-TV method has the best efficiency in terms of projector evaluations, independently from the penalty parameter $\alpha$, but has the disadvantage of solving a TV proximal problem in each iteration step which get harder to solve with increasing smoothing 
Table 2: Performance evaluation for different values of $\alpha$ (see Figure 3). The table displays the CPU time in seconds and required number of forward and backward projector evaluations $\left(K / K^{\mathrm{T}}\right)$ to get the relative error (67) below the error tolerance $\epsilon=0.05$. For each $\alpha$, the algorithms were run using the following parameters: FB-EM-TV $(\delta=0.1)$, FB-EM-TV-Nes83 $(\delta=0.1, \eta=0.5)$, CP-E $(\sigma=0.07)$, CP-SI $(\delta=0.1, \sigma=0.05)$, PIDSplit+ $(\gamma=10)$, which were chosen based on the "best" performance regarding $K / K^{\mathrm{T}}$ for $\epsilon=0.05$ in Table 1 .

\begin{tabular}{lcclcccccc}
\hline & \multicolumn{2}{c}{$\alpha=0.04$} & & \multicolumn{2}{c}{$\alpha=0.08$} & & \multicolumn{2}{c}{$\alpha=0.2$} \\
\cline { 2 - 3 } & $K / K^{\mathrm{T}}$ & $\mathrm{CPU}$ & & $K / K^{\mathrm{T}}$ & $\mathrm{CPU}$ & & $K / K^{\mathrm{T}}$ & $\mathrm{CPU}$ \\
\hline FB-EM-TV & 28 & 16.53 & & 20 & 40.55 & & $\mathbf{1 9}$ & 105.37 \\
FB-EM-TV-Nes83 & $\mathbf{1 7}$ & $\mathbf{5 . 2 6}$ & & $\mathbf{1 5}$ & 14.68 & & - & - \\
CP-E & 61 & 6.02 & & 48 & $\mathbf{4 . 7 9}$ & & 51 & $\mathbf{5 . 0 9}$ \\
CP-SI & - & - & & 25 & 23.73 & & 21 & 133.86 \\
PIDSplit+ & 32 & 8.08 & & 30 & 7.51 & & 38 & 9.7 \\
\hline
\end{tabular}

level (i.e. larger $\alpha$ ) leading to a negative computational time. The latter observation holds also for the CP-SI algorithm. In case of a rough approximation accuracy (see Table 2), the FB-EM-TV-Nes83 scheme is able to improve the overall performance, respectively at least the computational time for higher accuracy in Table 3 , but here the damping parameter $\eta$ in 22 has to be chosen carefully to ensure the convergence (cf. Table 2 and 3 in case of $\alpha=0.2$ ). Additionally based on Table 1 , a proper choice of $\eta$ is not only dependent on $\alpha$ but also on the inner accuracy of TV proximal problems.

- In contrast to FB-EM-TV and CP-SI, the remaining algorithms provide a superior computational time due to the solution of TV related steps by the shrinkage formula but show a strongly increased requirements on projector evaluations across all penalty parameters $\alpha$. In addition, the performance of these algorithms is strongly dependent on the proper setting of free parameters ( $\sigma$ in case of CP-E and $\gamma$ in PIDSplit+) which unfortunately are able to achieve only a fast initial convergence or a fast asymptotic convergence. Thus different parameter settings of $\sigma$ and $\gamma$ were used in Tables 2 and 3 .

\subsection{Spectral X-Ray CT}

Conventional X-ray CT is based on recording changes in the X-ray intensity due to attenuation of X-ray beams traversing the scanned object and has been applied in clinical practice for decades. However, the transmitted X-rays carry more information than just intensity changes since the attenuation of an X-ray depends strongly on its energy [2, 103]. It is well understood that the transmitted energy spectrum contains valuable information about the structure and material composition of the imaged object and can be utilized to better distinguish different types of absorbing material, such as varying tissue types or contrast agents. But the detectors employing in traditional CT systems provide an integral measure of absorption over the transmitted energy spectrum and thus eliminate spectral information [38, 151]. Even so, spectral information can be obtained by using different input spectra [72, 184] or using the concept of dual-layer (integrating) detectors [39]. This has been limited the practical usefulness of 
Table 3: Performance evaluation for different values of $\alpha$ (see Figure 3 ) as in Table 2 but for $\epsilon=0.005$ and using the following parameters: FB-EM-TV $(\delta=0.005)$, FB-EM-TV-Nes83 $(\delta=0.005, \eta=0.05)$, CP-E $(\sigma=0.2)$, CP-SI $(\delta=0.005, \sigma=0.3)$, PIDSplit+ $(\gamma=3)$.

\begin{tabular}{lccccccccc}
\hline & \multicolumn{2}{c}{$\alpha=0.04$} & & \multicolumn{2}{c}{$\alpha=0.08$} & & \multicolumn{2}{c}{$\alpha=0.2$} \\
\cline { 2 - 3 } & $K / K^{\mathrm{T}}$ & CPU & & $K / K^{\mathrm{T}}$ & $\mathrm{CPU}$ & & $K / K^{\mathrm{T}}$ & $\mathrm{CPU}$ \\
\hline FB-EM-TV & $\mathbf{2 7 6}$ & 2452.14 & & $\mathbf{1 6 8}$ & 4999.71 & & $\mathbf{1 7 5}$ & 12612.7 \\
FB-EM-TV-Nes83 & 512 & 222.98 & & 231 & 308.74 & & - & - \\
CP-E & 962 & $\mathbf{9 4 . 5 7}$ & & 696 & $\mathbf{6 9 . 8 6}$ & & 658 & $\mathbf{6 5 . 4 2}$ \\
CP-SI & 565 & 1117.12 & & 456 & 1427.71 & & 561 & 7470.18 \\
PIDSplit+ & 932 & 239.35 & & 698 & 179.77 & & 610 & 158.94 \\
\hline
\end{tabular}

energy-resolving imaging, also referred to as spectral CT, to dual energy systems. Recent advances in detector technology towards binned photon-counting detectors have enabled a new generation of detectors that can measure and analyze incident photons individually [38] providing the availability of more than two spectral measurements. This development has led to a new imaging method named K-edge imaging [107] that can be used to selectively and quantitatively image contrast agents loaded with K-edge materials [70, 130]. For a compact overview on technical and practical aspects of spectral CT we refer to [38, 151].

Two strategies have been proposed to reconstruct material specific images from spectral CT projection data and we refer to [151] for a compact overview. Either of them is a projectionbased material decomposition with a subsequent image reconstruction. This means that in the first step, estimates of material-decomposed sinograms are computed from the energy-resolved measurements, and in the second step, material images are reconstructed from the decomposed material sinograms. A possible decomposition method to estimate the material sinograms $f_{l}$, $l=1, \ldots, L$, from the acquired data is a maximum-likelihood estimator assuming a Poisson noise distribution [143], where $L$ is the number of materials considered. An accepted noise model for line integrals $f_{l}$ is a multivariate Gaussian distribution [151, 152] leading to a penalized weighted least squares (PWLS) estimator to reconstruct material images $u_{l}$ :

$$
\frac{1}{2}\left\|f-\left(I_{L} \otimes K\right) u\right\|_{\Sigma^{-1}}^{2}+\alpha R(u) \rightarrow \min _{u}, \quad \alpha>0,
$$

where $f=\left(f_{1}^{\mathrm{T}}, \ldots, f_{L}^{\mathrm{T}}\right)^{\mathrm{T}}, u=\left(u_{1}^{\mathrm{T}}, \ldots, u_{L}^{\mathrm{T}}\right)^{\mathrm{T}}, I_{L}$ denotes the $L \times L$ identity matrix, $\otimes$ represents the Kronecker product, and $K$ is the forward projection operator. The given block matrix $\Sigma$ is the covariance matrix representing the (multivariate) Gaussian distribution, where the off-diagonal block elements describe the inter-sinogram correlations, and can be estimated, e.g., using the inverse of the Fisher information matrix [142, 152]. Since $f$ is computed from a common set of measurements, the correlation of the decomposed data is very high and thus a significant improvement can in fact be expected intuitively by exploiting the fully populated covariance matrix $\Sigma$ in (68). In the following, we exemplary show reconstruction results on spectral CT data where (68) was solved by a proximal ADMM algorithm with a material independent total variation penalty function $R$ as discussed in [148]. For a discussion why ADMM based methods are more preferable for PWLS problems in X-ray CT than, e.g., gradient descent based techniques, we refer to [138]. 


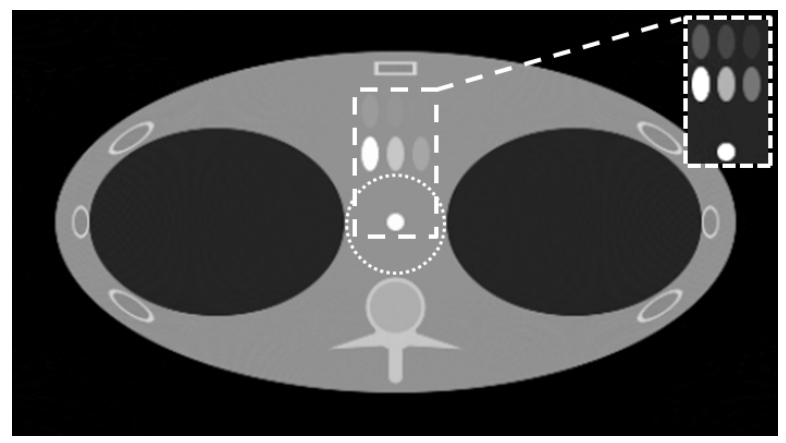

Figure 9: Software thorax phantom comprising sternum, ribs, lungs, vertebrae, and one circle and six ellipsoids containing different concentrations of K-edge material ytterbium [130]. The phantom was used to simulate spectral CT measurements with a six-bin photon-counting detector.

Figures 10 and 11 show an example for a statistical image reconstruction method applied to K-edge imaging. A numerical phantom as shown in Figure 9 was employed in a spectral CT simulation study assuming a photon-counting detector. Using an analytical spectral attenuation model, spectral measurements were computed. The assumed X-ray source spectrum and detector response function of the photon-counting detector were identical to those employed in a prototype spectral CT scanner described in [153]. The scan parameters were set to tube voltage $130 \mathrm{kVp}$, anode current $200 \mu \mathrm{A}$, detector width/height 946.38/1.14 mm, number of columns 1024, source-to-isocenter/-detector distance 570/1040 mm, views per turn 1160, time per turn $1 \mathrm{~s}$, and energy thresholds to $25,46,61,64,76$, and $91 \mathrm{keV}$. The spectral data were then decomposed into 'photo-electric absorption', 'Compton effect', and 'ytterbium' by performing a maximum-likelihood estimation [143]. By computing the covariance matrix $\Sigma$ of the material decomposed sinograms via the Fisher information matrix [142, 152] and treating the sinograms as the mean and $\Sigma$ as the variance of a Gaussian random vector, noisy material sinograms were computed. Figures 10 and 11 show material images that were then reconstructed using the traditional filtered backprojection (upper row) and proximal ADMM algorithm as described in [148] (middle and lower row). In the latter case, two strategies were performed: (1) keeping only the diagonal block elements of $\Sigma$ in (68) and thus neglecting crosscorrelations and decoupling the reconstruction of material images (middle row); (2) using the fully populated covariance matrix $\Sigma$ in (68) such that all material images have to be reconstructed jointly (lower row). The results suggest, best visible in the K-edge images in Figure 11, that the iterative reconstruction method, which exploits knowledge of the inter-sinogram correlations, produces images that possess a better reconstruction quality. For comparison of iterative reconstruction strategies, the regularization parameters were chosen manually so the reconstructed images possessed approximately the same variance within the region indicated by the dotted circle in Figure 9. Further (preliminary) results that demonstrate advantages of exploiting inter-sinogram correlations on computer-simulated and experimental data in spectral CT can be found in [148, 180].

Acknowledgements The authors thank Frank Wübbeling (University of Münster, Germany) for providing the Monte-Carlo simulation for the synthetic 2D PET data. The authors also thank Thomas Koehler (Philips Technologie GmbH, Innovative Technologies, Hamburg, 

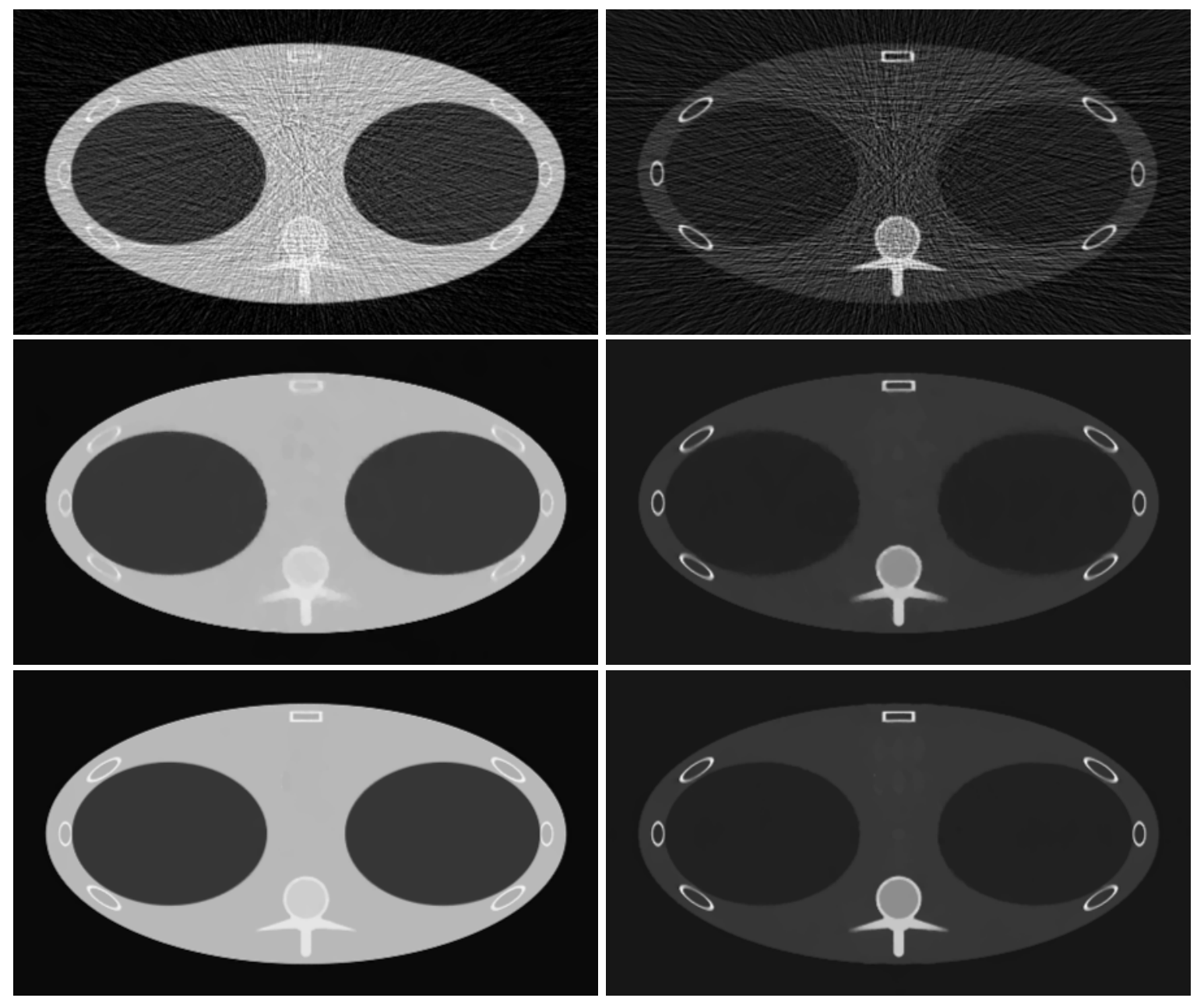

Figure 10: Reconstructions based on the thorax phantom (see Figure 90 using the traditional filtered backprojection with Shepp-Logan filter (upper row) and a proximal ADMM algorithm as described in [148. (middle and lower row). The middle row shows results based on (68) neglecting cross-correlations between the material decomposed sinograms and lower row using the fully populated covariance matrix $\Sigma$. The material images show the results for the 'Compton effect' (left column) and 'photo-electric absorption' (right column). The K-edge material 'ytterbium' is shown in Figure 11. 


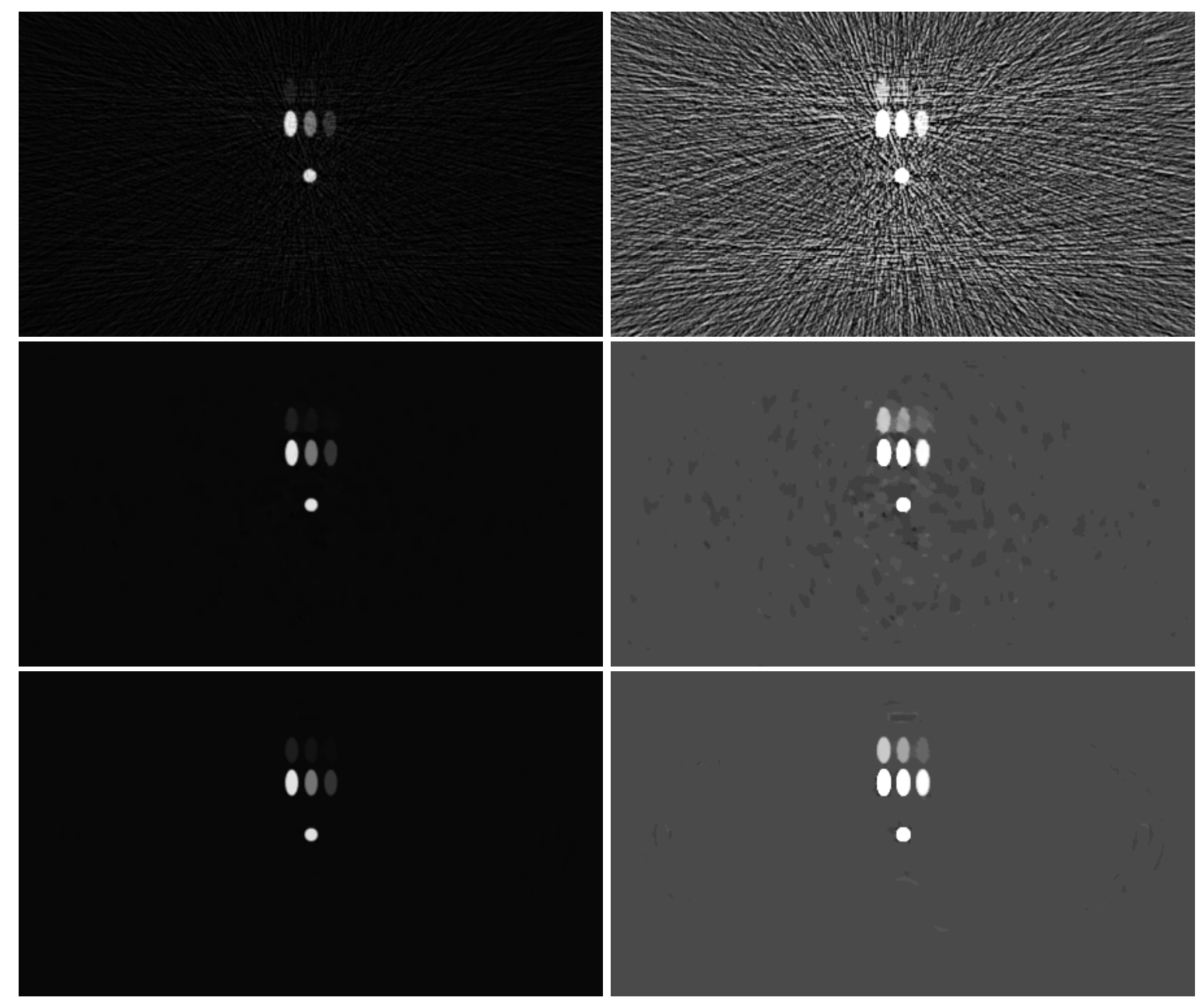

Figure 11: Reconstructions of the K-edge material 'ytterbium' using the thorax phantom shown in Figure 9. For details see Figure 10. To recognize the differences, the maximal intensity value of original reconstructed images shown in left column was set down in the right column. 
Germany) for comments that improved the manuscript. The work on spectral CT was performed when A. Sawatzky was with the Computational Bioimaging Laboratory at Washington University in St. Louis, USA, and was supported in part by NIH award EB009715 and funded from Philips Research North America.

\section{References}

[1] J. F. P.-J. Abascal, J. Chamorro-Servent, J. Aguirre, S. Arridge, T. Correia, J. Ripoll, J. J. Vaquero, and M. Desco. Fluorescence diffuse optical tomography using the split Bregman method. Med. Phys., 38:6275, 2011.

[2] R. E. Alvarez and A. Macovski. Energy-selective reconstructions in X-ray computerized tomography. Phys. Med. Biol., 21(5):733-744, 1976.

[3] S. Anthoine, J.-F. Aujol, Y. Boursier, and C. Mélot. On the efficiency of proximal methods in CBCT and PET. In Proc. IEEE Int. Conf. Image Proc. (ICIP), pages 1365-1368, 2011.

[4] S. Anthoine, J.-F. Aujol, Y. Boursier, and C. Mélot. Some proximal methods for CBCT and PET tomography. In Proc. SPIE (Wavelets and Sparsity XIV), volume 8138, 2011.

[5] K. J. Arrow, L. Hurwitz, and H. Uzawa. Studies in Linear and Nonlinear Programming. Stanford University Press, 1958.

[6] H. Attouch and J. Bolte. On the convergence of the proximal algorithm for nonsmooth functions involving analytic features. Math. Program., 116(1-2):5-16, 2009.

[7] H. Attouch, J. Bolte, and B. F. Svaiter. Convergence of descent methods for semialgebraic and tame problems: proximal algorithms, forward-backward splitting, and regularized Gauss-Seidel methods. Math. Program. Series A, 137(1-2):91-129, 2013.

[8] J.-P. Aubin. Optima and Equilibria: An Introduction to Nonlinear Analysis. Springer, Berlin, Heidelberg, New York, 2nd edition, 2003.

[9] M. Bachmayr and M. Burger. Iterative total variation schemes for nonlinear inverse problems. Inverse Problems, 25(10):105004, 2009.

[10] E. Bae, J. Yuan, and X.-C. Tai. Global minimization for continuous multiphase partitioning problems using a dual approach. Int. J. Comput. Vision, 92(1):112-129, 2011.

[11] J. Barzilai and J. M. Borwein. Two-point step size gradient methods. IMA J. Numer. Anal., 8(1):141-148, 1988.

[12] H. H. Bauschke and P. L. Combettes. Convex Analysis and Monotone Operator Theory in Hilbert Spaces. Springer, New York, 2011.

[13] A. Beck and M. Teboulle. Fast gradient-based algorithms for constrained total variation image denoising and deblurring. SIAM J. Imag. Sci., 2:183-202, 2009.

[14] S. Becker, J. Bobin, and E. J. Candès. NESTA: A fast and accurate first-order method for sparse recovery. SIAM J. Imag. Sci., 4(1):1-39, 2011. 
[15] M. Benning, L. Gladden, D. Holland, C.-B. Schönlieb, and T. Valkonen. Phase reconstruction from velocity-encoded MRI measurement - A survey of sparsity-promoting variational approaches. J. Magn. Reson., 238:26-43, 2014.

[16] M. Benning, P. Heins, and M. Burger. A solver for dynamic PET reconstructions based on forward-backward-splitting. In AIP Conf. Proc., volume 1281, pages 1967-1970, 2010 .

[17] D. P. Bertsekas. Constrained Optimization and Lagrange Multiplier Methods. Academic Press, New York, 1982.

[18] D. P. Bertsekas. Incremental proximal methods for large scale convex optimization. Math. Program., Ser. B, 129(2):163-195, 2011.

[19] J. M. Bioucas-Dias and M. A. T. Figueiredo. Multiplicative noise removal using variable splitting and constrained optimization. IEEE Trans. Image Process., 19(7):1720-1730, 2010 .

[20] A. Björck. Least Squares Problems. SIAM, Philadelphia, 1996.

[21] D. Boley. Local linear convergence of the alternating direction method of multipliers on quadratic or linear programs. SIAM J. Optim., 2014.

[22] J. Bolte, S. Sabach, and M. Teboulle. Proximal alternating linearized minimization for nonconvex and nonsmooth problems. Math. Program., Series A, 2013.

[23] S. Bonettini and V. Ruggiero. On the convergence of primal-dual hybrid gradient algorithms for total variation image restoration. J. Math. Imaging Vis., 44:236-253, 2012.

[24] J. F. Bonnans, J. C. Gilbert, C. Lemaréchal, and C. A. Sagastizábal. A family of variable metric proximal methods. Mathematical Programming, 68:15-47, 1995.

[25] R. I. Bot and C. Hendrich. A Douglas-Rachford type primal-dual method for solving inclusions with mixtures of composite and parallel-sum type monotone operators. SIAM J. Optim., 23(4):2541-2565, 2013.

[26] R. I. Bot and C. Hendrich. Convergence analysis for a primal-dual monotone + skew splitting algorithm with applications to total variation minimization. J. Math. Imaging Vis., 49(3):551-568, 2014.

[27] S. Boyd, N. Parikh, E. Chu, B. Peleato, and J. Eckstein. Distributed optimization and statistical learning via the alternating direction method of multipliers. Foundations and Trends in Machine Learning, 3(1):101-122, 2011.

[28] K. Bredies, K. Kunisch, and T. Pock. Total generalized variation. SIAM J. Imag. Sci., $3(3): 492-526,2010$.

[29] F. E. Browder and W. V. Petryshyn. The solution by iteration of nonlinear functional equations in Banach spaces. Bull. Am. Math. Soc., 72:571-575, 1966.

[30] C. Brune, A. Sawatzky, and M. Burger. Primal and dual Bregman methods with application to optical nanoscopy. Int. J. Comput. Vision, 92(2):211-229, 2010. 
[31] M. Burger and S. Osher. A guide to the TV zoo. In Level Set and PDE Based Reconstruction Methods in Imaging, pages 1-70. Springer, 2013.

[32] M. Burger, E. Resmerita, and L. He. Error estimation for Bregman iterations and inverse scale space methods in image restoration. Computing, 81(2-3):109-135, 2007.

[33] J. V. Burke and M. Qian. A variable metric proximal point algorithm for monotone operators. SIAM J. Control Optim., 37:353-375, 1999.

[34] F. Büther, M. Dawood, L. Stegger, F. Wübbeling, M. Schäfers, O. Schober, and K. P. Schäfers. List mode-driven cardiac and respiratory gating in PET. J. Nucl. Med., 50(5):674-681, 2009.

[35] D. Butnariu and A. N. Iusem. Totally convex functions for fixed points computation and infinite dimensional optimization, volume 40 of Applied Optimization. Kluwer, Dordrecht, 2000.

[36] C. Byrne. A unified treatment of some iterative algorithms in signal processing and image reconstruction. Inverse Problems, 20:103-120, 2004.

[37] J.-F. Cai, E. J. Candès, and Z. Shen. A singular value thresholding algorithm for matrix completion. SIAM J. Optim., 20(4):1956 - 1982, 2010.

[38] J. Cammin, J. S. Iwanczyk, and K. Taguchi. Emerging Imaging Technologies in Medicine, chapter Spectral/Photo-Counting Computed Tomography, pages 23-39. CRC Press, 2012.

[39] R. Carmi, G. Naveh, and A. Altman. Material separation with dual-layer CT. In Proc. IEEE Nucl. Sci. Symp. Conf. Rec., pages 1876-1878, 2005.

[40] A. Chambolle, V. Caselles, D. Cremers, M. Novaga, and T. Pock. An introduction to total variation for image analysis. In Theoretical Foundations and Numerical Methods for Sparse Recovery, volume 9 of Radon Series Compl. Appl. Math., pages 263-340. Walter de Gruyter, 2010.

[41] A. Chambolle and T. Pock. Diagonal preconditioning for first order primal-dual algorithms in convex optimization. Proc. IEEE Int. Conf. Comput. Vision (ICCV), pages $1762-1769,2011$.

[42] A. Chambolle and T. Pock. A first-order primal-dual algorithm for convex problems with applications to imaging. J. Math. Imaging Vis., 40(1):120-145, 2011.

[43] R. Chartrand, E. Y. Sidky, and X. Pan. Nonconvex compressive sensing for X-ray CT: an algorithm comparison. In Asilomar Conference on Signals, Systems, and Computers, pages 665-669, 2013.

[44] C. Chaux, M. EI-Gheche, J. Farah, J. Pesquet, and B. Popescu. A parallel proximal splitting method for disparity estimation from multicomponent images under illumination variation. J. Math. Imaging Vis., 47(3):1-12, 2012.

[45] G. Chen and M. Teboulle. A proximal-based decomposition method for convex minimization problems. Math. Program., 64:81-101, 1994. 
[46] G. H.-G. Chen and R. T. Rockafellar. Convergence rates in forward-backward splitting. SIAM J. Optim., 7:421-444, 1997.

[47] G. Chierchia, N. Pustelnik, J.-C. Pesquet, and B. Pesquet-Popescu. Epigraphical projection and proximal tools for solving constrained convex optimization problems: Part I. Technical report, 2013. http://arxiv.org/abs/1210.5844.

[48] K. Choi, J. Wang, L. Zhu, T.-S. Suh, S. Boyd, and L. Xing. Compressed sensing based cone-beam computed tomography reconstruction with a first-order method. Med. Phys., 37(9):5113-5125, 2010.

[49] E. Chouzenoux, J.-C. Pesquet, and A. Repetti. Variable metric forward-backward algorithm for minimizing the sum of a differentiable function and a convex function. $J$. Optim. Theory Appl., pages 107-132, 2013.

[50] P. Combettes and J.-C. Pesquet. Proximal splitting methods in signal processing. In Fixed-Point Algorithms for Inverse Problems in Science and Engineeering, pages 185212. Springer, 2011.

[51] P. Combettes and J.-C. Pesquet. Primal-dual splitting algorithm for solving inclusions with mixture of composite, Lipschitzian, and parallel-sum type monotone operators. Set-Valued and Variational Analysis, 20(2):307-330, 2012.

[52] P. L. Combettes. Solving monotone inclusions via compositions of nonexpansive averaged operators. Optimization, 53(5-6):475-504, 2004.

[53] P. L. Combettes and J.-C. Pesquet. Proximal thresholding algorithm for minimization over orthonormal bases. SIAM J. Optim., 18(4):1351-1376, 2007.

[54] P. L. Combettes and B. C. Vu. Variable metric forward-backward splitting with applications to monotone inclusions in duality. Optimization, pages 1-30, 2012.

[55] L. Condat. A primal-dual splitting method for convex optimization involving Lipschitzian, proximable and linear composite terms. J. Optim. Theory Appl., 158(2):460479, 2013.

[56] W. Cong, J. Yang, and G. Wang. Differential phase-contrast interior tomography. Phys. Med. Biol., 57:2905-2914, 2012.

[57] J. Dahl, P. C. Hansen, S. H. Jensen, and T. L. Jensen. Algorithms and software for total variation image reconstruction via first-order methods. Numer. Algor., 53(1):6792,2010 .

[58] I. Daubechies, M. Defrise, and C. De Mol. An iterative thresholding algorithm for linear inverse problems with a sparsity constraint. Commun. Pure Appl. Math., 51:1413-1541, 2004 .

[59] I. Daubechies, M. Fornasier, and I. Loris. Accelerated projected gradient methods for linear inverse problems with sparsity constraints. J. Fourier Anal. Appl., 14(5-6):764792, 2008. 
[60] C. Davis. All convex invariant functions of Hermitian matrices. Archive in Mathematics, 8:276-278, 1957.

[61] N. Dey, L. Blanc-Feraud, C. Zimmer, P. Roux, Z. Kam, J.-C. Olivo-Marin, and J. Zerubia. Richardson-Lucy algorithm with total variation regularization for $3 \mathrm{D}$ confocal microscope deconvolution. Microsc. Res. Tech., 69:260-266, 2006.

[62] J. Duchi, S. Shalev-Shwartz, Y. Singer, and T. Chandra. Efficient projections onto the 11-ball for learning in high dimensions. In ICML '08 Proceedings of the 25th International Conference on Machine Learning, ACM New York, 2008.

[63] J. Eckstein and D. P. Bertsekas. An alternating direction method for linear programming. Tech. Report MIT Lab. for Info. and Dec. Sys., 1990.

[64] J. Eckstein and D. P. Bertsekas. On the Douglas-Rachford splitting method and the proximal point algorithm for maximal monotone operators. Math. Program., 55:293 $318,1992$.

[65] E. Esser. Applications of Lagrangian-based alternating direction methods and connections to split Bregman. UCLA CAM Report 09-31, 2009.

[66] E. Esser, X. Zhang, and T. F. Chan. A general framework for a class of first order primal-dual algorithms for convex optimization in imaging science. SIAM J Imag Sci, 3(4):1015-1046, 2010.

[67] F. Facchinei and J.-S. Pang. Finite-Dimensional Variational Inequalities and Complementarity Problems, volume II. Springer, New York, 2003.

[68] J. A. Fessler. Conjugate-gradient preconditioning methods: Numerical results. Technical Report 303, Commun. Signal Process. Lab., Dept. Elect. Eng. Comput. Sci., Univ. Michigan, Ann Arbor, MI, Jan. 1997. available from http://web.eecs.umich.edu/ $\sim$ fessler/.

[69] J. A. Fessler and S. D. Booth. Conjugate-gradient preconditioning methods for shiftvariant PET image reconstruction. IEEE Trans. Image Process., 8(5):688-699, 1999.

[70] S. Feuerlein, E. Roessl, R. Proksa, G. Martens, O. Klass, M. Jeltsch, V. Rasche, H.-J. Brambs, M. H. K. Hoffmann, and J.-P. Schlomka. Multienergy photon-counting Kedge imaging: Potential for improved luminal depiction in vascular imaging. Radiology, 249(3):1010-1016, 2008.

[71] M. Figueiredo and J. Bioucas-Dias. Deconvolution of Poissonian images using variable splitting and augmented Lagrangian optimization. In IEEE/SP Workshop on Statistical Signal Processing, pages 733-736, 2009.

[72] T. G. Flohr, C. H. McCollough, H. Bruder, M. Petersilka, K. Gruber, C. Süß, M. Grasruck, K. Stierstorfer, B. Krauss, R. Raupach, A. N. Primak, A. Küttner, S. Achenbach, C. Becker, A. Kopp, and B. M. Ohnesorge. First performance evaluation of a dual-source CT (DSCT) system. Eur. Radiol., 16:256-268, 2006.

[73] M. Fornasier. Theoretical Foundations and Numerical Methods for Sparse Recovery, volume 9. Walter de Gruyter, 2010. 
[74] G. Frassoldati, L. Zanni, and G. Zanghirati. New adaptive stepsize selections in gradient methods. J. Ind. Manage. Optim., 4(2):299-312, 2008.

[75] M. Freiberger, C. Clason, and H. Scharfetter. Total variation regularization for nonlinear fluorescence tomography with an augmented Lagrangian splitting approach. Appl. Opt., 49(19):3741-3747, 2010.

[76] K. Frick, P. Marnitz, and A. Munk. Statistical multiresolution estimation for variational imaging: With an application in Poisson-biophotonics. J. Math. Imaging Vis., 46:370$387,2013$.

[77] D. Gabay. Applications of the method of multipliers to variational inequalities. In M. Fortin and R. Glowinski, editors, Augmented Lagrangian Methods: Applications to the Solution of Boundary Value Problems, chapter IX, pages 299-340. North-Holland, Amsterdam, 1983.

[78] D. Gabay and B. Mercier. A dual algorithm for the solution of nonlinear variational problems via finite element approximations. Comput. Math. Appl., 2:17-40, 1976.

[79] H. Gao, S. Osher, and H. Zhao. Mathematical Modeling in Biomedical Imaging II: Optical, Ultrasound, and Opto-Acoustic Tomographies, chapter Quantitative Photoacoustic Tomography, pages 131-158. Springer, 2012.

[80] H. Gao, H. Yu, S. Osher, and G. Wang. Multi-energy CT based on a prior rank, intensity and sparsity model (PRISM). Inverse Problems, 27(11):115012, 2011.

[81] R. Glowinski and P. Le Tallec. Augmented Lagrangian and Operator-Splitting Methods in Nonlinear Mechanics, volume 9 of SIAM Studies in Applied and Numerical Mathematics. SIAM, Philadelphia, 1989.

[82] R. Glowinski and A. Marroco. Sur l'approximation, par éléments finis d'ordre un, et la résolution, par pénalisation-dualité d'une classe de problèmes de Dirichlet non linéaires. Revue française d'automatique, informatique, recherche opérationnelle. Analyse numérique, 9(2):41-76, 1975.

[83] D. Goldfarb and K. Scheinberg. Fast first-order methods for composite convex optimization with line search. SIAM J. Imag. Sci., 2011.

[84] T. Goldstein, X. Bresson, and S. Osher. Geometric applications of the split Bregman method: Segmentation and surface reconstruction. J. Sci. Comput., 45:272-293, 2010.

[85] T. Goldstein and S. Osher. The split Bregman method for L1-regularized problems. SIAM J. Imag. Sci., 2(2):323-343, 2009.

[86] B. Goris, W. Van den Broek, K. J. Batenburg, H. H. Mezerji, and S. Bals. Electron tomography based on a total variation minimization reconstruction technique. Ultramicroscopy, 113:120-130, 2012.

[87] O. Güler. New proximal point algorithms for convex minimization. SIAM J. Optim., 2(4):649-664, 1992. 
[88] S. Harizanov, J.-C. Pesquet, and G. Steidl. Epigraphical projection for solving least squares Anscombe transformed constrained optimization problems. In A. K. et al., editor, Scale-Space and Variational Methods in Computer Vision. Lecture Notes in Computer Science, SSVM 2013, LNCS 7893, pages 125-136, Berlin, 2013. Springer.

[89] B. He, L.-Z. Liao, D. Han, and H. Yang. A new inexact alternating directions method for monotone variational inequalities. Math. Program., Ser. A, 92(1):103-118, 2002.

[90] B. He and H. Yang. Some convergence properties of the method of multiplieres for linearly constrained monotone variational operators. Operation Research Letters, 23:151161, 1998.

[91] B. He and X. Yuan. On the $\mathcal{O}(1 / n)$ convergence rate of the Douglas-Rachford alternating direction method. SIAM J. Numer. Anal., 2:700-709, 2012.

[92] B. S. He, H. Yang, and S. L. Wang. Alternating direction method with self-adaptive penalty parameters for monotone variational inequalties. J. Optimiz. Theory App., 106(2):337-356, 2000.

[93] S. W. Hell. Toward fluorescence nanoscopy. Nat. Biotechnol., 21(11):1347-1355, 2003.

[94] S. W. Hell. Far-field optical nanoscopy. Science, 316(5828):1153-1158, 2007.

[95] M. R. Hestenes. Multiplier and gradient methods. J. Optim. Theory Appl., 4:303-320, 1969 .

[96] M. Hong and Z. Q. Luo. On linear convergence of the alternating direction method of multipliers. Arxiv preprint 1208.3922, 2012.

[97] J. Huang, S. Zhang, and D. Metaxas. Efficient MR image reconstruction for compressed MR imaging. Med. Image Anal., 15:670-679, 2011.

[98] X. Jia, Y. Lou, R. Li, W. Y. Song, and S. B. Jiang. GPU-based fast cone beam CT reconstruction from undersampled and noisy projection data via total variation. Med. Phys., 37(4):1757-1760, 2010.

[99] B. Kaltenbacher, A. Neubauer, and O. Scherzer. Iterative regularization methods for nonlinear ill-posed problems, volume 6. Walter de Gruyter, 2008.

[100] S. H. Kang, B. Shafei, and G. Steidl. Supervised and transductive multi-class segmentation using $p$-Laplacians and RKHS methods. J. Visual Commun. Image Represent., 25(5):1136-1148, 2014.

[101] K. C. Kiwiel. Free-steering relaxation methods for problems with strictly convex costs and linear constraints. Math. Oper. Res., 22(2):326-349, 1997.

[102] K. C. Kiwiel. Proximal minimization methods with generalized Bregman functions. SIAM J. Control Opt., 35(4):1142-1168, 1997.

[103] G. F. Knoll. Radiation Detection and Measurement. Wiley, 3rd edition, 2000. 
[104] N. Komodakis and J.-C. Pesquet. Playing with duality: an overview of recent primal-dual approaches for solving large-scale optimization problems. ArXiv Preprint arXiv:1406.5429, 2014.

[105] S. Kontogiorgis and R. R. Meyer. A variable-penalty alternating directions method for convex optimization. Math. Program., 83(1-3):29-53, 1998.

[106] M. A. Krasnoselskii. Two observations about the method of successive approximations. Uspekhi Matematicheskikh Nauk, 10:123-127, 1955. In Russian.

[107] R. A. Kruger, S. J. Riederer, and C. A. Mistretta. Relative properties of tomography, K-edge imaging, and K-edge tomography. Med. Phys., 4(3):244-249, 1977.

[108] J. Lellmann, J. Kappes, J. Yuan, F. Becker, and C. Schnörr. Convex multi-class image labeling with simplex-constrained total variation. In X.-C. Tai, K. Morken, M. Lysaker, and K.-A. Lie, editors, Scale Space and Variational Methods, volume 5567 of LNCS, volume 5567 of Lecture Notes in Computer Science, pages 150-162. Springer, 2009.

[109] P. L. Lions and B. Mercier. Splitting algorithms for the sum of two linear operators. SIAM J. Num. Anal., 16:964-976, 1979.

[110] M. Lustig, D. Donoho, and J. M. Pauly. Sparse MRI: The application of compressed sensing for rapid MR imaging. Magn. Reson. Med., 58:1182-1195, 2007.

[111] S. Ma, W. Y. Y. Zhang, and A. Chakraborty. An efficient algorithm for compressed MR imaging using total variation and wavelets. In Proc. IEEE Comput. Vision Pattern Recognit., pages 1-8, 2008.

[112] P. Machart, S. Anthoine, and L. Baldassarre. Optimal computational tradeoff of inexact proximal methods. Technical report, arXiv e-print, 2012. http://arxiv.org/abs/1210.5034.

[113] W. R. Mann. Mean value methods in iteration. Proceedings of the American Mathematical Society, 16(4):506-510, 1953.

[114] B. Martinet. Régularisation d'inéquations variationnelles par approximations successives. Revue Française d'Informatique et de Recherche Operationelle, 4(3):154-158, 1970.

[115] A. Mehranian, A. Rahmim, M. R. Ay, F. Kotasidis, and H. Zaidi. An ordered-subsets proximal preconditioned gradient algorithm for edge-preserving PET image reconstruction. Med. Phys., 40(5):052503, 2013.

[116] J. Müller, C. Brune, A. Sawatzky, T. Kösters, F. Wübbeling, K. Schäfers, and M. Burger. Reconstruction of short time PET scans using Bregman iterations. In Proc. IEEE Nucl. Sci. Symp. Conf. Rec., pages 2383-2385, 2011.

[117] F. Natterer and F. Wübbeling. Mathematical Methods in Image Reconstruction. SIAM, 2001.

[118] A. S. Nemirovsky and D. B. Yudin. Problem Complexity and Method Efficiency in Optimization. J. Wiley \& Sons, Ltd., 1983. 
[119] Y. Nesterov. Introductory Lectures on Convex Optimization - A Basic Course, volume 87 of Applied Optimization. Springer US, 2004.

[120] Y. Nesterov. Gradient methods for minimizing composite functions. Math. Program., Series B, 140(1):125-161, 2013.

[121] Y. E. Nesterov. A method of solving a convex programming problem with convergence rate $\mathrm{O}\left(1 / k^{2}\right)$. Soviet Mathematics Doklady, 27(2):372-376, 1983.

[122] Y. E. Nesterov. Smooth minimization of non-smooth functions. Math. Program., 103:127-152, 2005.

[123] H. Nien and J. A. Fessler. Fast X-ray CT image reconstruction using the linearized augmented Lagrangian method with ordered subsets. Technical report, arXiv e-print, 2014. http://arxiv.org/abs/1402.4381.

[124] M. Nilchian, C. Vonesch, P. Modregger, M. Stampanoni, and M. Unser. Fast iterative reconstruction of differential phase contrast X-ray tomograms. Optics Express, 21(5):5511-5528, 2013.

[125] P. Ochs, Y. Chen, T. Brox, and T. Pock. iPiano: Inertial proximal algorithm for nonconvex optimization. SIAM J Imaging Sci, 7(2):1388-1419, 2014.

[126] Z. Opial. Weak convergence of a sequence of successive approximations for nonexpansive mappings. Bull. Am. Math. Soc., 73:591-597, 1967.

[127] J. M. Ortega and W. C. Rheinboldt. Iterative Solution of Nonlinear Equations in Several Variables. SIAM, New York, 1970.

[128] S. Osher, M. Burger, D. Goldfarb, J. Xu, and W. Yin. An iterative regularization method for the total variation based image restoration. Multiscale Model. Simul., 4:460489, 2005.

[129] S. Osher, M. Burger, D. Goldfarb, J. Xu, and W. Yin. An iterative regularization method for total variation-based image restoration. Multiscale Model. Simul., 4(2):460489, 2005.

[130] D. Pan, C. O. Schirra, A. Senpan, A. H. Schmieder, A. J. Stacy, E. Roessl, A. Thran, S. A. Wickline, R. Proksa, and G. M. Lanza. An early investigation of ytterbium nanocolloids for selective and quantitative "multicolor" spectral CT imaging. ACS Nano, 6(4):3364-3370, 2012.

[131] L. A. Parente, P. A. Lotito, and M. V. Solodov. A class of inexact variable metric proximal point algorithms. SIAM J. Optim., 19(1):240-260, 2008.

[132] N. Parikh and S. Boyd. Proximal algorithms. Foundations and Trends in Optimization, 1(3):123-231, 2013.

[133] T. Pock, A. Chambolle, D. Cremers, and H. Bischof. A convex relaxation approach for computing minimal partitions. IEEE Conf. Comput. Vision Pattern Recognit., pages 810-817, 2009. 
[134] M. J. D. Powell. A method for nonlinear constraints in minimization problems. Optimization, 1972.

[135] K. P. Pruessmann, M. Weiger, M. B. Scheidegger, and P. Boesiger. SENSE: Sensitivity encoding for fast MRI. Magn. Reson. Med., 42:952-962, 1999.

[136] N. Pustelnik, C. Chaux, J.-C. Pesquet, and C. Comtat. Parallel algorithm and hybrid regularization for dynamic PET reconstruction. In Proc. IEEE Nucl. Sci. Symp. Conf. Rec., pages 2423-2427, 2010.

[137] S. Ramani and J. A. Fessler. Parallel MR image reconstruction using augmented Lagrangian methods. IEEE Trans. Med. Imag., 30(3):694-706, 2011.

[138] S. Ramani and J. A. Fessler. A splitting-based iterative algorithm for accelerated statistical X-ray CT reconstruction. IEEE Trans. Med. Imag., 31(3):677-688, 2012.

[139] R. T. Rockafellar. Augmented Lagrangians and applications of the proximal point algorithm in convex programming. Math. Oper. Res., 1(2):97-116, 1976.

[140] R. T. Rockafellar. Monotone operators and the proximal point algorithm. SIAM J. Control Opt., 14:877-898, 1976.

[141] R. T. Rockafellar. Convex Analysis. Princeton University Press, Princeton, 10 edition, 1997.

[142] E. Roessl and C. Herrmann. Cramér-Rao lower bound of basis image noise in multipleenergy x-ray imaging. Phys. Med. Biol., 54(5):1307-1318, 2009.

[143] E. Roessl and R. Proksa. K-edge imaging in x-ray computed tomography using multi-bin photon counting detectors. Phys. Med. Biol., 52(15):4679-4696, 2007.

[144] L. Rudin, S. Osher, and E. Fatemi. Nonlinear total variation based noise removal algorithms. Physica D, 60:259-268, 1992.

[145] A. Sawatzky. Performance of first-order algorithms for TV penalized weighted leastsquares denoising problem. In Image and Signal Processing, volume 8509 of Lecture Notes in Computer Science, pages 340-349. Springer International Publishing, 2014.

[146] A. Sawatzky, C. Brune, T. Kösters, F. Wübbeling, and M. Burger. EM-TV methods for inverse problems with Poisson noise. In Level Set and PDE Based Reconstruction Methods in Imaging, pages 71-142. Springer, 2013.

[147] A. Sawatzky, D. Tenbrinck, X. Jiang, and M. Burger. A variational framework for region-based segmentation incorporating physical noise models. J. Math. Imaging Vis., 47(3):179-209, 2013.

[148] A. Sawatzky, Q. Xu, C. O. Schirra, and M. A. Anastasio. Proximal ADMM for multi-channel image reconstruction in spectral X-ray CT. IEEE Trans. Med. Imaging, 33(8):1657-1668, 2014.

[149] H. Schäfer. Über die Methode sukzessiver Approximationen. Jahresbericht der Deutschen Mathematiker-Vereinigung, 59:131-140, 1957. 
[150] K. P. Schäfers, T. J. Spinks, P. G. Camici, P. M. Bloomfield, C. G. Rhodes, M. P. Law, C. S. R. Baker, and O. Rimoldi. Absolute quantification of myocardial blood flow with $\mathrm{H}_{2}{ }^{15} \mathrm{O}$ and 3-dimensional PET: An experimental validation. J. Nucl. Med., 43:1031-1040, 2002.

[151] C. O. Schirra, B. Brendel, M. A. Anastasio, and E. Roessl. Spectral CT: a technology primer for contrast agent development. Contrast Media Mol. Imaging, 9(1):62-70, 2014.

[152] C. O. Schirra, E. Roessl, T. Koehler, B. Brendel, A. Thran, D. Pan, M. A. Anastasio, and R. Proksa. Statistical reconstruction of material decomposed data in spectral CT. IEEE Trans. Med. Imag., 32(7):1249-1257, 2013.

[153] J. P. Schlomka, E. Roessl, R. Dorscheid, S. Dill, G. Martens, T. Istel, C. Bäumer, C. Herrmann, R. Steadmann, G. Zeitler, A. Livne, and R. Proksa. Experimental feasibility of multi-energy photon-counting K-edge imaging in pre-clinical computed tomography. Phys. Med. Biol., 53(15):4031-4047, 2008.

[154] M. Schmidt, N. Le Roux, and F. Bach. Convergence rates of inexact proximalgradient methods for convex optimization. Technical report, arXiv e-print, 2011. http://arxiv.org/abs/1109.2415.

[155] M. Schrader, S. W. Hell, and H. T. M. van der Voort. Three-dimensional superresolution with a 4Pi-confocal microscope using image restoration. J. Appl. Phys., 84(8):4033-4042, 1998.

[156] T. Schuster, B. Kaltenbacher, B. Hofmann, and K. S. Kazimierski. Regularization methods in Banach spaces, volume 10. Walter de Gruyter, 2012.

[157] S. Setzer. Operator splittings, Bregman methods and frame shrinkage in image processing. Int. J. Comput. Vision, 92(3):265-280, 2011.

[158] S. Setzer, G. Steidl, and J. Morgenthaler. A cyclic projected gradient method. Comput. Optim. Appl., 54(2):417-440, 2013.

[159] S. Setzer, G. Steidl, and T. Teuber. Deblurring Poissonian images by split Bregman techniques. J. Vis. Commun. Image R., 21:193-199, 2010.

[160] E. Y. Sidky, J. H. Jörgensen, and X. Pan. Convex optimization problem prototyping for image reconstruction in computed tomography with the Chambolle-Pock algorithm. Phys. Med. Biol., 57(10):3065-3091, 2012.

[161] G. Steidl and T. Teuber. Removing multiplicative noise by Douglas-Rachford splitting methods. J. Math. Imaging Vis., 36:168-184, 2010.

[162] T. Teuber. Anisotropic Smoothing and Image Restoration Facing Non-Gaussian Noise. $\mathrm{PhD}$ thesis, Technische Universität Kaiserslautern, Apr. 2012. available from https: //kluedo.ub.uni-kl.de/frontdoor/index/index/docId/3219.

[163] P. Tseng. Applications of a splitting algorithm to decomposition in convex programming and variational inequalities. SIAM J. Control Opt., 29:119-138, 1991. 
[164] P. Tseng. On accelerated proximal gradient methods for convex-concave optimization. 2008. http://www.eecs.berkeley.edu/ brecht/eecs227cdocs/tseng.pdf.

[165] T. Valkonen. A primal-dual hybrid gradient method for nonlinear operators with applications to MRI. Inverse Problems, 30(5):055012, 2014.

[166] B. Vandeghinste, B. Goossens, J. D. Beenhouwer, A. Pizurica, W. Philips, S. Vandenberghe, and S. Staelens. Split-Bregman-based sparse-view CT reconstruction. Proc. Int. Meeting Fully 3D Image Recon. Rad. Nucl. Med., pages 431-434, 2011.

[167] Y. Vardi, L. A. Shepp, and L. Kaufman. A statistical model for positron emission tomography. J. Am. Stat. Assoc., 80(389):8-20, 1985.

[168] S. Villa, S. Salzo, L. Baldassarre, and A. Verri. Accelerated and inexact forwardbackward algorithms. SIAM J. Optim., 23(3):1607-1633, 2013.

[169] J. von Neumann. Some matrix inequalities and metrization of matrix-space. In Collected Works, Pergamon, Oxford, 1962, Volume IV, 205-218, pages 286-300. Tomsk University Review, 1937.

[170] B. C. Vũ. A splitting algorithm for dual monotone inclusions involving cocoercive operators. Adv. Comput. Math., 38(3):667-681, 2013.

[171] G. Wang, H. Yu, and B. D. Man. An outlook on x-ray CT research and development. Med. Phys., 35(3):1051-1064, 2008.

[172] J. Wang, T. Li, H. Lu, and Z. Liang. Penalized weighted least-squares approach to sinogram noise reduction and image reconstruction for low-dose X-ray computed tomography. IEEE Trans. Med. Imag., 25(10):1272-1283, 2006.

[173] K. Wang, R. Su, A. A. Oraevsky, and M. A. Anastasio. Investigation of iterative image reconstruction in three-dimensional optoacoustic tomography. Phys. Med. Biol., 57:5399-5423, 2012.

[174] S. L. Wang and L. Z. Liao. Decomposition method with a variable parameter for a class of monotone variational inequality problems. J. Optimiz. Theory App., 109(2):415-429, 2001.

[175] G. A. Watson. Characterization of the subdifferential of some matrix norms. Linear Algebra Appl., 170:33-45, 1992.

[176] M. N. Wernick and J. N. Aarsvold, editors. Emission Tomography: The Fundamentals of PET and SPECT. Elsevier Academic Press, 2004.

[177] Q. Xu, A. Sawatzky, and M. A. Anastasio. A multi-channel image reconstruction method for grating-based X-ray phase-contrast computed tomography. In Proc. SPIE 9033, Medical Imaging 2014: Physics of Medical Imaging, 2014.

[178] Q. Xu, A. Sawatzky, M. A. Anastasio, and C. O. Schirra. Sparsity-regularized image reconstruction of decomposed K-edge data in spectral CT. Phys. Med. Biol., 59(10):N65, 2014 . 
[179] J. Yuan, C. Schnörr, and G. Steidl. Simultaneous higher order optical flow estimation and decomposition. SIAM J. Sci. Comput., 29(6):2283-2304, 2007.

[180] R. Zhang, J.-B. Thibault, C. A. Bouman, and K. D. S. J. Hsieh. A model-based iterative algorithm for dual-energy X-ray $\mathrm{CT}$ reconstruction. In Proc. Int. Conf. Image Form. in X-ray $C T$, pages 439-443, 2012.

[181] X. Zhang, M. Burger, and S. Osher. A unified primal-dual algorithm framework based on Bregman iteration. J. Sci. Comput., 46(1):20-46, 2011.

[182] M. Zhu and T. F. Chan. An efficient primal-dual hybrid gradient algorithm for total vatiation image restoration. UCLA CAM Report 08-34, 2008.

[183] H. Zou and T. Hastie. Regularization and variable selection via the elastic net. Journal of Royal Statistical Socienty: Series B (Statisctical Methodology), 67:301-320, 2005.

[184] Y. Zou and M. D. Silver. Analysis of fast kV-switching in dual energy CT using a pre-reconstruction decomposition technique. In Proc. SPIE (Medical Imaging 2008), volume 6913, page 691313, 2008. 